I N T ER N ATIONAL MONETARY FUND

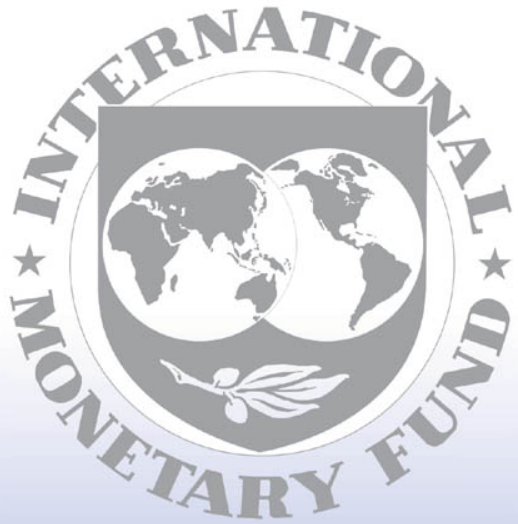

Staff

Country

Reports 


\title{
Seychelles: Third Review Under the Stand-By Arrangement, Financing Assurances Review, Cancellation of the Stand-By-Arrangement, and Request for an Extended Arrangement- Staff Report; Staff Statement, Press Release on the Executive Board Discussion; and Statement by the Executive Director for Seychelles
}

In the context of the Third Review Under the Stand-By Arrangement, Financing Assurances Review, Cancellation of the Stand-By-Arrangement, and Request for an Extended Arrangement, the following documents have been released and are included in this package:

- $\quad$ The staff report for Third Review Under the Stand-By Arrangement, Financing Assurances Review, Cancellation of the Stand-By-Arrangement, and Request for an Extended Arrangement, prepared by a staff team of the IMF, following discussions that ended on November 2, 2009, with the officials of Seychelles on economic developments and policies. Based on information available at the time of these discussions, the staff report was completed on December 4, 2009. The views expressed in the staff report are those of the staff team and do not necessarily reflect the views of the Executive Board of the IMF.

- A staff statement of December 18, 2009, updating information on recent economic developments.

- $\quad$ A Press Release summarizing the views of the Executive Board as expressed during its December 18, 2009, discussion of the staff report that completed the review.

- A statement by the Executive Director for Seychelles.

The documents listed below have been separately released.

Letter of Intent sent to the IMF by the authorities of Seychelles *

Memorandum of Economic and Financial Policies by the authorities of Seychelles*

Technical Memorandum of Understanding*

*Also included in Staff Report

The policy of publication of staff reports and other documents allows for the deletion of marketsensitive information.

\author{
Copies of this report are available to the public from \\ International Monetary Fund • Publication Services \\ $70019^{\text {th }}$ Street, N.W. • Washington, D.C. 20431 \\ Telephone: (202) 623-7430 • Telefax: (202) 623-7201 \\ E-mail: publications@imf.org • Internet: http://www.imf.org
}

\section{International Monetary Fund Washington, D.C.}


INTERNATIONAL MONETARY FUND

\title{
SEYCHELLES
}

\section{Third Review Under the Stand-By Arrangement, Financing Assurances Review, Cancellation of the Stand-By-Arrangement, and Request for an Extended Arrangement}

\author{
Prepared by the African Department \\ (In consultation with other Departments) \\ Approved by Roger Nord and Aasim Husain
}

December 4, 2009

The program is on track and macroeconomic stabilization has advanced rapidly. The authorities continue to implement the program with a high degree of ownership and success. All quantitative performance criteria (PCs) and structural benchmarks at end-September 2009 were met. To date, SDR 8.8 million (100 percent of quota) has been disbursed under the two-year Stand-By Arrangement (SBA) and SDR 2.2 million (25 percent of quota) is available upon completion of the third Stand-By Arrangement review.

Policy discussions were held in Victoria Oct. 18-Nov. 2, 2009. Staff met with President James Michel, Minister of Finance Danny Faure, Governor Pierre Laporte of the Central Bank of Seychelles (CBS), other senior government officials, and representatives of parliament, the private sector, civil society, and the diplomatic community. The mission comprised Paul Mathieu (head), and included Marie-Therese Trasino, Nikoloz Gigineishvili (all AFR), Patrick Imam (MCM), and Nkunde Mwase (SPR).

In the attached letter from President Michel and accompanying Memorandum of Economic and Financial Policies (MEFP) for 2010-12, the authorities request completion of the third program review under the Stand-By Arrangement and a new three-year arrangement under the Extended Fund Facility (EFF) for 225 percent of quota (SDR19.8 million). The program objectives are to support the public debt restructuring process aimed at reestablishing external sustainability; accompany the second generation structural reforms aimed at securing macroeconomic stability and raising growth performance; and to put in place a macroeconomic framework to guide policies through 2012.

Program design: EFF program reviews are proposed on a semi-annual basis, with quarterly performance criteria and structural benchmarks, financing assurances reviews (as necessary), and purchases. Structural reform focuses on: (i) improving PFM; (ii) reforming the tax system; (iii) redefining the role and raising the performance of para-public entities; and (iv) bolstering the financial system.

It is proposed that the third Stand-By Arrangement review be completed, the Stand-By Arrangement canceled, and the new EFF arrangement approved. 
Executive Summary $\underline{3}$

I. Macroeconomic Developments and Performance Under the SBA

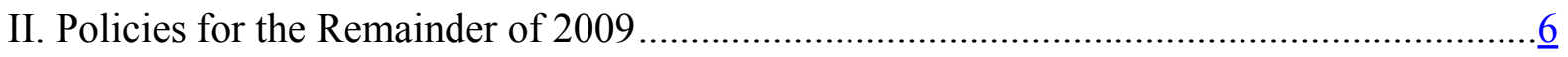

III. Medium-Term Macroeconomic Policy and Reform Efforts......................................... $\underline{6}$

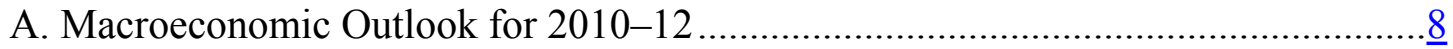

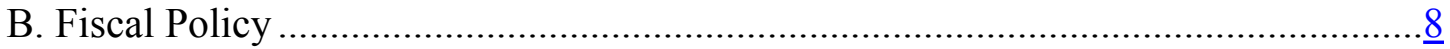

C. Monetary, Exchange, and Financial Sector Policies ......................................... 9

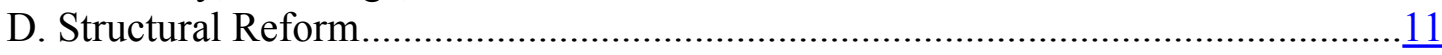

IV. Program Financing, Design, Monitoring, and Risks ….......................................11

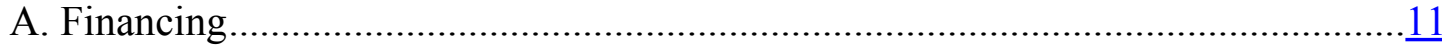

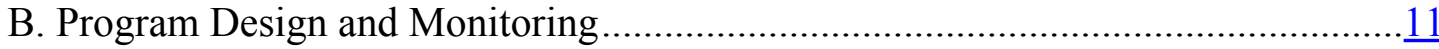

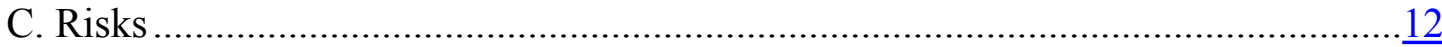

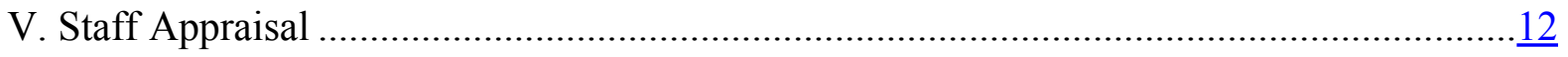

Tables

1. Selected Economic and Financial Indicators, 2007-12 ..........................................14

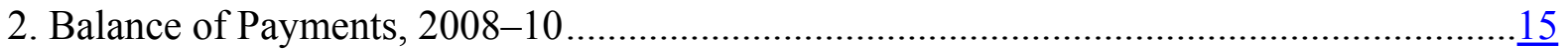

3. Consolidated Government Operations, 2007-12 .....................................................

4. Monetary Survey and Central Bank Accounts, 2007-10 ..............................................18

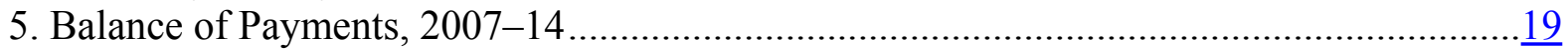

6. Financial Soundness Indicators for the Banking Sector, 2007-09 ................................ $\frac{20}{21}$

7. Projected Financing Requirements and Sources, 2008-19 .........................................

8. Fund Disbursements and Timing of Reviews Under the Proposed

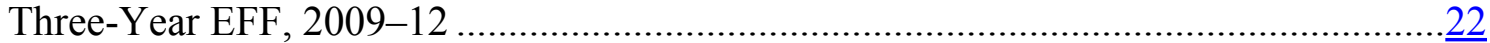

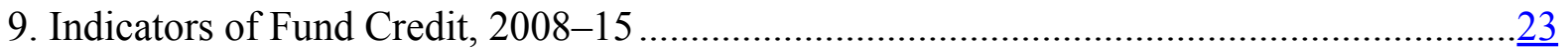

Figures

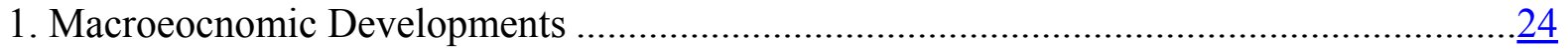

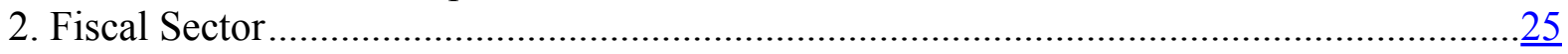

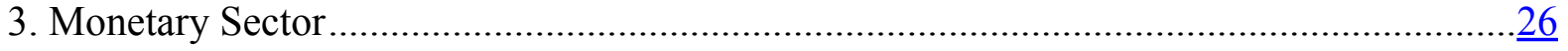

Appendices

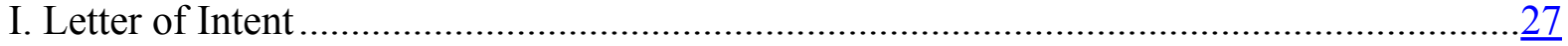

Attachment 1. Memorandum of Economic, Financial and Structural Reform Policies

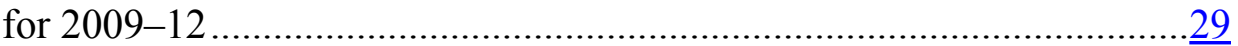

Attachment 2. Technical Memorandum of Understanding......................................... $\frac{\overline{51}}{16}$

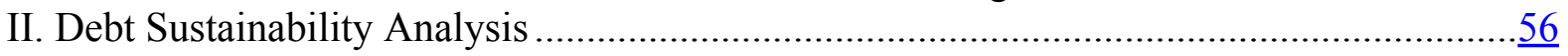




\section{EXECUTIVE SUMMARY}

- Macroeconomic performance through September 2009 continued to be encouraging. The liberalization of the exchange regime, a strong fiscal adjustment, and prudent monetary policies have contributed to restoring macroeconomic stability and setting the stage for recovery. Monthly inflation has been near zero since March and the exchange rate has appreciated steadily from lows in early 2009. Short term interest rates on government securities declined sharply from about 30 percent in January to below 5 percent in October reflecting strong deflation and fiscal tightening. Real GDP is now expected to decline by 7.5 percent in 2009 , somewhat better than previously feared, as the economy responds to the reforms and improving global conditions and the threat of piracy is being addressed.

- The structural reform effort is progressing well. The structural benchmarks for end-September were met and those for end-December are also expected to be met. Key progress has been made on public financial management, notably through the treasury single account. The 2010 budget features a much improved and complete presentation of government finance. Substantial progress is also being made in strengthening accountability and governance in the public enterprise sector.

- Discussions on the restructuring Seychelles' public external debt have advanced markedly. A formal exchange offer to commercial bondholders is expected to be launched by mid-December. The exchange offer has the support of the African Development Bank in the form of a partial guarantee. Good progress on non-Paris Club bilaterals and commercial bank debt has also been made. The authorities' debt strategy is consistent with the Fund's LIA policy.

- The reform successes of 2009 set the stage for a coherent medium-term effort to extend the gains for growth and stability. The November 2008 Stand-By Arrangement addressed the foreign exchange distortions, implemented a fiscal adjustment, and stabilized the macroeconomic environment, while taking first steps toward much-needed structural reform. With these objectives achieved, the stage is set for the second generation of reforms to secure macroeconomic stability and improve economic performance. The success of Seychelles' transformation effort requires that a sound medium-term macroeconomic framework be in place to guide policy through the next few years.

- A three-year EFF arrangement is the most appropriate instrument to support an ambitious structural transformation. Moreover with public debt restructuring discussions at a critical juncture and the Fund's commitment of an appropriate multiyear arrangement would add needed credibility to the authorities' efforts to reestablish fiscal and public debt sustainability. 


\section{Macroeconomic Developments ANd Performance Under the SBA}

\section{Macroeconomic performance through September 2009 continued to be} encouraging and the reform process was pursued with a high degree of ownership and determination. The liberalization of the exchange regime, a strong fiscal adjustment, and prudent monetary policies have contributed to restoring macroeconomic stability and setting the stage for recovery. Monthly inflation has been near zero since March and the exchange rate has appreciated steadily from lows in early 2009. Short-term interest rates on government securities declined sharply from about 30 percent in January to below 5 percent in October reflecting strong deflation and fiscal tightening. Fiscal policy has over performed and the primary surplus reached 13.4 percent of GDP over the first nine months, on strong revenue

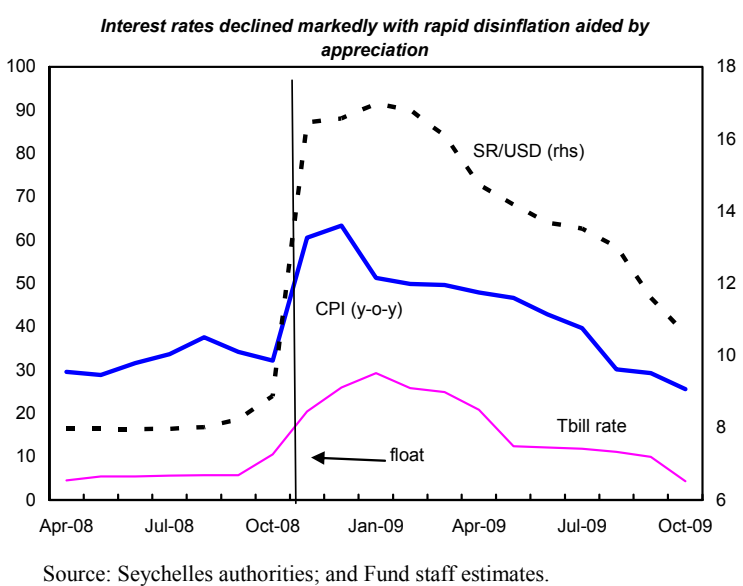

Source: Seychelles authorities; and Fund staff estimates. performance, tight expenditure control, and one off factors.

\section{The updated external outlook indicates a much better than expected outturn}

for 2009. Real GDP is now expected to decline by 7.5 percent in 2009 , somewhat better than previously feared, as the economy responds to the reforms and improving global conditions and the threat of piracy is being addressed (Table 1). The external current account deficit has narrowed sharply during the first three quarters of 2009, reflecting lower imports, better than expected service income, and higher grants. The decrease in world food and oil prices together with a slowdown in tourism and FDI have reduced import demand. Official external reserves have been rebuilt from near depleted levels to US\$134 million (1.3 months of imports) at endSeptember 2009 on emerging confidence in the rupee. The SDR allocation was also been fully added to reserves. All quantitative PCs at end-September 2009 were met with margins (MEFP, Table 1).

\section{Rapid disinflation in $\mathbf{2 0 0 9}$ has} provided room for a measured easing of monetary policy. Following the float, the
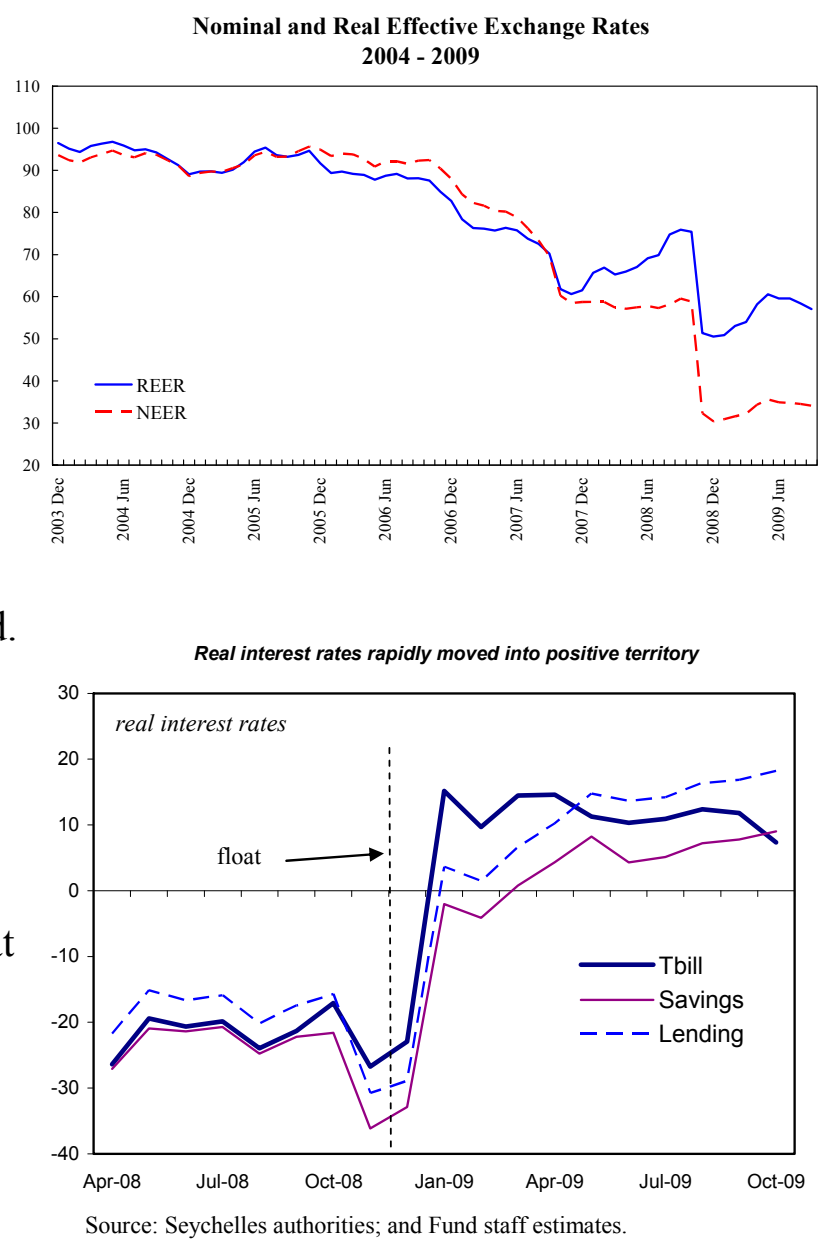
rupee has overshot, but has since appreciated partly due to very high positive real interest rates, but also because banks were unloading large open foreign exchange positions. The CBS has been successful in striking a right balance between disinflation objective and ensuring orderly conditions in the foreign exchange market helping banks with portfolio adjustments. Importantly, the local asset requirement was progressively reduced and finally abolished in August.
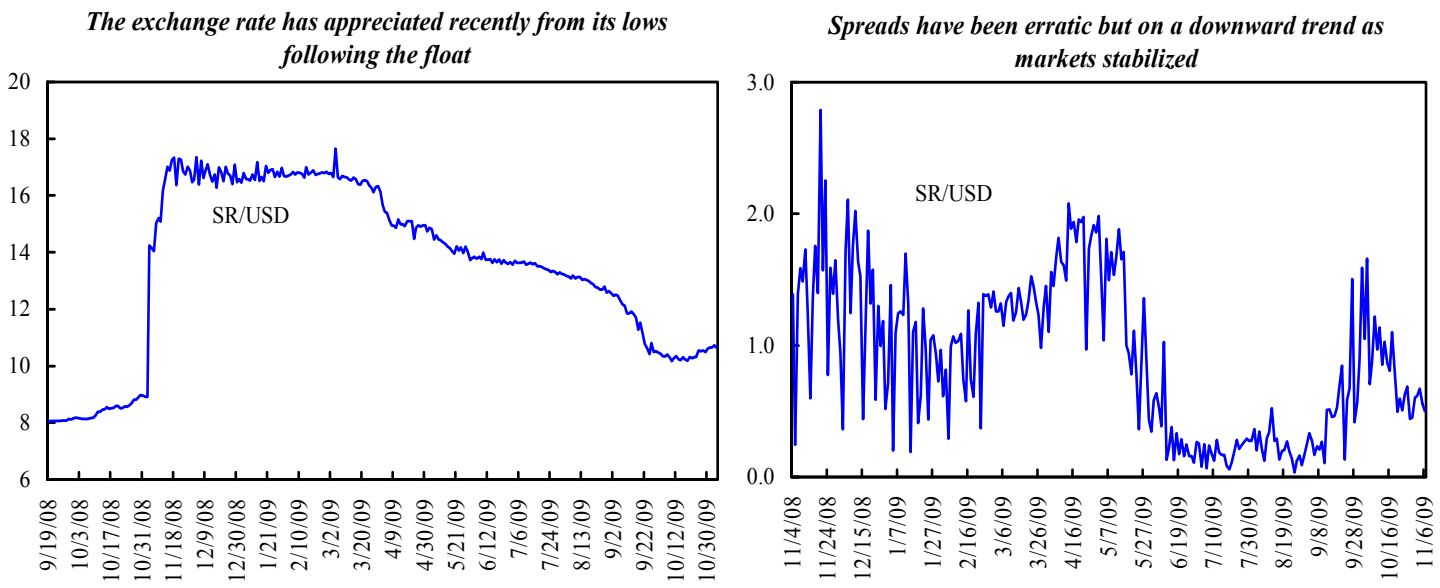

Source: Seychelles authorities; and Fund staff estimates

4. The structural reform effort is progressing well (MEFP, Table 2). The three structural benchmarks for end-September were met: the treasury single account was introduced and is leveraging progress in public financial management (PFM); the CBS developed and adopted foreign reserve management guidelines; and the Public Enterprise Monitoring and Control Act was passed by National Assembly. Moreover, the end-December structural benchmarks are also expected to be met (MEFP, Table 3). External financial audits of the seven largest public enterprises were completed and management audits of major public entities have also advanced. Based on the results of these audits, the management of the public utilities company was sacked in August and a management and technical assistance contract signed with a foreign expert firm. Reform action plans for several other firms are in various stages of elaboration. Financial reporting obligations to the Finance Ministry have been put in place supported by the new public enterprise control act, and corporate governance guidelines have been issued. The authorities have also adopted a public sector action plan, with World Bank support, to guide expenditure reforms, based on public expenditure reviews (PER) of the key health and education sectors.

\section{Discussions on the restructuring Seychelles' public external debt have advanced} markedly. Following agreement with Paris Club creditors in April 2009, one non-Paris Club bilateral agreement has been signed (Malaysia) and agreements are close with several others. Agreements on some commercial bank loans have also been reached. A formal exchange offer to commercial bondholders is expected to be launched by mid-December. The exchange offer has the support of the African Development Bank in the form of a Policybased Guarantee Operation (approved December 2). ${ }^{1}$ The authorities feel that the guarantee

\footnotetext{
${ }^{1}$ The authorities' request for a US\$10 million Policy-based Guarantee Operation, the first of its kind under the new AfDB facility, has taken longer that expected to process. The guarantee, in response to creditor feedback,
} 
considerably strengthens the appeal of the offer and its chances of success by considerably lowering credit risk on the new bonds. Fitch Ratings has been asked to rate the new bonds. Staff is of the view that debt restructuring negotiations are proceeding in a manner consistent with the Fund's LIA policy.

\section{Policies for the Remainder OF 2009}

6. The outlook for the remainder of 2009 is for inflation to remain in the low single digits, with emerging growth impulses (MEFP, $\mid 11-14)$. The external current account deficit is expected to narrow, with exports of tourism services projected to increase as the global economy recovers and additional hotel accommodation comes on line (Table 2). Gross reserves are expected to rise further to US\$153 million by end-2009 (1.5 months of imports).

7. The 2009 primary surplus is now projected to reach $\mathbf{1 3 . 4}$ percent of GDP, in line with the adjusted performance target for end-December (Table 3 and MEFP, Table 1). The over performance in the first three quarters has provided the fiscal space for a supplementary appropriation of about 2 percent of GDP to meet emergency and strategic expenditure needs. Fiscal performance, coupled with falling interest rates and increased external financial assistance, will reduce domestic debt by 51/2 percent of GDP in 2009. Treasury management capacity is being strengthened in close collaboration with the CBS.

8. Monetary policy will focus on consolidating low inflation while providing sufficient room for credit to the private sector. Inflation has remained near zero for some time and the exchange rate appreciation of recent months is still working its way through domestic prices. The reserve money and reserves targets for December have been raised moderately to provide increasing space for credit to the private sector and facilitate financial deepening (Table 4).

\section{Medium-Term Macroeconomic Policy And Reform EfForts}

9. The reform successes of $\mathbf{2 0 0 9}$ set the stage for a coherent medium-term effort to extend the gains for growth and stability (MEFP, \15-18). Seychelles' economic and financial crisis of 2008 reflected a decade's long pursuit of unsustainable policies and rising structural distortions. The November 2008 Stand-By Arrangement addressed the foreign exchange distortions, implemented a fiscal adjustment, and stabilized the macroeconomic environment, while taking first steps toward much-needed structural reform. With these objectives achieved, the stage is set for the second generation of reforms to secure macroeconomic stability and improve economic performance. The ambitious reforms ahead aim to transform the economy by strengthening public financial management (PFM), institutionalizing higher governance standards, rationalizing the public sector, reforming

would cover 2-3 semi-annual payments on a rolling basis. While small relative to the size of the exchange bond, the guarantee, costing some 50 basis points, would add a nonnegligible improvement of the external debt profile. 
taxation, bolstering the financial system, and improving the business environment (Text Table 1).

Text Table 1. Seychelles: Structural Benchmarks for 2009-10

\begin{tabular}{|c|c|c|c|}
\hline Measure & Target date & Macroeconomic rationale & Status \\
\hline $\begin{array}{l}\text { Introduce a treasury single } \\
\text { account. }\end{array}$ & $\begin{array}{l}\text { End- } \\
\text { September } 2009\end{array}$ & $\begin{array}{l}\text { To strengthen public } \\
\text { finances, key to sustainability } \\
\text { efforts. }\end{array}$ & $\begin{array}{l}\text { Met. The TSA is } \\
\text { leveraging progress on } \\
\text { PFM. }\end{array}$ \\
\hline $\begin{array}{l}\text { Adopt foreign reserves } \\
\text { management investment } \\
\text { guidelines. }\end{array}$ & $\begin{array}{l}\text { End- } \\
\text { September } 2009\end{array}$ & $\begin{array}{l}\text { To ensure management of } \\
\text { foreign reserves in line with } \\
\text { best international practice. }\end{array}$ & $\begin{array}{l}\text { Met. The CBS Board } \\
\text { adopted guidelines on } \\
\text { September } 25,2009 \text {. }\end{array}$ \\
\hline $\begin{array}{l}\text { Adopt Public Enterprise } \\
\text { Monitoring and Control act. }\end{array}$ & $\begin{array}{l}\text { End- } \\
\text { September } 2009\end{array}$ & $\begin{array}{l}\text { To improve oversight over } \\
\text { public enterprises and reduce } \\
\text { quasi-fiscal risks. }\end{array}$ & $\begin{array}{l}\text { Met. Parliamentary } \\
\text { approval secured on } \\
\text { September 28, } 2009 .\end{array}$ \\
\hline $\begin{array}{l}\text { Complete CBS procedures } \\
\text { manual (MEFP, } \llbracket 46) \text {. }\end{array}$ & $\begin{array}{l}\text { End- } \\
\text { December } 2009\end{array}$ & $\begin{array}{l}\text { To support improved } \\
\text { efficiency and transparency } \\
\text { in monetary policy. }\end{array}$ & In progress. \\
\hline $\begin{array}{l}\text { CBS to publish commercial bank } \\
\text { supervision report (MEFP, } \uparrow 46 \text { ). }\end{array}$ & $\begin{array}{l}\text { End- } \\
\text { December } 2009\end{array}$ & $\begin{array}{l}\text { To promote competition and } \\
\text { transparency in the banking } \\
\text { system. }\end{array}$ & In progress. \\
\hline $\begin{array}{l}\text { Amend the Business Tax Act in } \\
\text { line with the tax reform strategy } \\
\text { (MEFP, }(22) \text {. }\end{array}$ & $\begin{array}{l}\text { End- } \\
\text { December } 2009\end{array}$ & $\begin{array}{l}\text { To broaden the tax base, } \\
\text { modernize tax policy, and } \\
\text { remove distortions. }\end{array}$ & In progress. \\
\hline $\begin{array}{l}\text { Cabinet approval of customs } \\
\text { reform strategy and } \\
\text { implementation plan (MEFP, } \\
\text { \23). }\end{array}$ & End-March 2010 & $\begin{array}{l}\text { To transform customs into a } \\
\text { modern and efficient entity. }\end{array}$ & \\
\hline $\begin{array}{l}\text { Publish general government } \\
\text { fiscal statistics (MEFP, } \llbracket 29 \text { ). }\end{array}$ & End-April 2010 & $\begin{array}{l}\text { To enhance transparency } \\
\text { and coverage of public } \\
\text { finance. }\end{array}$ & \\
\hline $\begin{array}{l}\text { Submit to National Assembly a } \\
\text { new customs management act } \\
\text { (MEFP, } \mid 23 \text { ). }\end{array}$ & End-June 2010 & $\begin{array}{l}\text { To institutionalize best } \\
\text { international practice and a } \\
\text { better business climate. }\end{array}$ & \\
\hline $\begin{array}{l}\text { Introduce Personal Income Tax } \\
\text { (MEFP, \22). }\end{array}$ & July 1,2010 & $\begin{array}{l}\text { To broader the tax base and } \\
\text { provide for more equitable } \\
\text { taxation. }\end{array}$ & \\
\hline $\begin{array}{l}\text { Introduce budget submissions } \\
\text { protocols and procedures } \\
\text { (MEFP, ๆ28). }\end{array}$ & End-July 2010 & $\begin{array}{l}\text { To strengthen budget } \\
\text { preparation. }\end{array}$ & \\
\hline $\begin{array}{l}\text { Adopt a new chart of account for } \\
\text { the } 2011 \text { budget (MEFP, } \llbracket 29 \text { ). }\end{array}$ & $\begin{array}{l}\text { End- } \\
\text { November } 2010\end{array}$ & $\begin{array}{l}\text { To ensure proper } \\
\text { classification and increase } \\
\text { efficiency of the budget as a } \\
\text { policy tool. }\end{array}$ & \\
\hline $\begin{array}{l}\text { Submit to National Assembly a } \\
\text { bill creating a national clearing } \\
\text { house and settlement system } \\
\text { (MEFP, ๆ44). }\end{array}$ & $\begin{array}{l}\text { End- } \\
\text { December } 2010\end{array}$ & $\begin{array}{l}\text { To introduce necessary } \\
\text { financial infrastructure for } \\
\text { improved efficiency and } \\
\text { reduced risks. }\end{array}$ & \\
\hline
\end{tabular}


10. The success of Seychelles' transformation effort requires that a sound mediumterm macroeconomic framework is in place to guide policy through the next few years. A three-year EFF arrangement is the most appropriate instrument to support an ambitious structural transformation. Moreover with public debt restructuring discussions at a critical juncture and the Fund's commitment of an appropriate multi-year arrangement would add needed credibility to the authorities' efforts to reestablish fiscal and public debt sustainability.

\section{A. Macroeconomic Outlook for 2010-12}

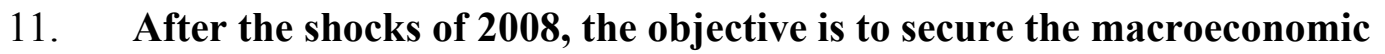
stabilization and implement policies designed to improve economic efficiency and durably raise growth. Tourism and related services - the main drivers of growth - are beginning to recover from a sharp decline, and FDI should rebound in 2010, with several large projects in the wings. The investment rebound would be supported by a successful public external debt restructuring in early 2010 that would reduce country credit risk. Real GDP growth is projected to recover to 4 percent in 2010 and to 5 percent in 2011-12, while inflation is expected to remain in the low single digits.

\section{The program objective is to reach a sustainable medium-term external path} from 2010 onwards (MEFP, ๆ33-35) This will depend to a great extent on achieving an appropriate public external debt restructuring. Under program macroeconomic policies the external current account deficit is expected to widen in 2010, due to higher growth and FDI, but would narrow over the medium term as tourism earnings recover (Table 5). Financing is secured for 2009, while moderate residual gaps remain for 2010-11, after assumed debt restructuring, which could be largely covered by access under the proposed EFF.

\section{An updated DSA indicates that public external debt remains unsustainable} without additional relief to that granted by Paris Club creditors (Appendix II). Under an alternative scenario that assumes that all other creditors agree to restructure Seychelles' public external debt on terms comparable to those granted by the Paris Club, external financing gaps would be closed (with the proposed additional Fund access) and the public external debt stock would be set on a sustainable path. Staff has followed closely the authorities efforts to ensure the terms offered are consistent with the program's financing and debt sustainability objectives. The second tranche of debt relief from Paris Club creditors is conditioned on completion of the first EFF review and a comparability of treatment test. Bound tests show that, even after a restructuring Seychelles remains vulnerable to shocks.

\section{B. Fiscal Policy}

\section{The overarching goal of fiscal policy is to place public finances on a firmly} sustainable footing (MEFP, \19-21) This will be achieved through a major reinforcement of fiscal monitoring and control and improved expenditure efficiency, while effecting a strong rise in public investment, which has been neglected during the recent crisis years. Significant primary surpluses will be targeted through the medium term, although at levels lower than the unsustainably high outturn of 2009 , to allow a reduction of domestic debt and provide space for private sector growth. The medium-term fiscal profile is consistent with the objective of putting fiscal policy on a sustainable path. 
15. A lower primary surplus is programmed for 2010-12, made possible by rapid macrostabilization and reductions in the interest bill. While the tax reform is targeted to be broadly revenue neutral over the medium term, in 2010 the net impact is an estimated positive 0.3 percent of GDP, largely on account of clawbacks of exemptions. However, a large reduction in tax-to-GDP ratio in 2010 is expected, reflecting one-off positive factors in 2009 and the nominal appreciation of the rupee (about 22 percent through late November), totaling about $4 \frac{1}{2}-5$ percent of GDP. Expenditure rationalization and efforts to reduce the size of the government will create room for critical capital investments in infrastructure; primary spending would rise by about 1 percent of GDP. Given the risks to the revenue projections, the 2010 budget will also include a back loaded expenditure contingency of about 1 percent of GDP comprising identified investment spending, to be used as long as the primary balance remains on track. Should overall revenue exceed projections in 2010, a portion of the over performance will be saved. The appropriate savings/spending balance would be determined at the time of the first program review under the EFF, determined in the context of the macroeconomic situation then prevailing. A further drawdown in domestic debt is targeted.

\section{The 2010 budget will introduce a medium-term fundamental reform of tax} policy to harmonize and lower rates, remove distortions, and improve compliance (MEFP, \22-23). The reform is meant to strengthen the business environment, ensure a more equitable spread of the burden, level the playing field and reduce the taxation of labor. A flat rate personal income tax on salaries, interest and dividend income will be introduced midyear and extended, at a lower rate, to previously untaxed non residents and expatriate workers. The business tax rate will be significantly lowered, offset in part by eliminating the corporate profit threshold, the incorporation of previously concessionary sectors into the tax base, and the introduction of an excise tax regime. ${ }^{2}$ With Fund technical assistance support, the authorities are reinforcing tax and customs administration. Additional technical assistance is being sought to support the introduction of the value-added tax in 2012 .

17. The 2010 budget will include a much fuller policy discussion and presentation of general government fiscal operations as well as an annual borrowing plan backed by a medium-term debt strategy (MEFP, q24-29). The strengthening of PFM and expenditure rationalization is under way, informed by World Bank sectoral PERs. Further downsizing of government noncore employment through a major outsourcing for noncore staff, as well as a targeted voluntary departure scheme, will strengthen efficiency. A new wage grid, designed to improve remuneration transparency and the retention of higher skilled civil servants, introduced with the 2010 budget is a major a step forward.

\section{Monetary, Exchange, and Financial Sector Policies}

\section{Monetary policy will aim at maintaining inflation in the low single digits} (MEFP, $\mid 37-47$ ). The CBS will continue to use reserve money targeting as an operational framework, which has gained further flexibility with the introduction of new indirect policy instruments. Repurchase operations are gradually becoming the main policy tool to guide

\footnotetext{
${ }^{2}$ The business tax rate is being lowered from $40 \%$ to $33 / 25 \%$ (banks and other corporates) in 2010 with the intension to lower it further over time.
} 
short-term interest rates. A credit auction arrangement has been introduced, and starting in 2010, a standing deposit facility will also become operational. Interest rates will need to remain positive in real terms, and the CBS should stand ready to react quickly to any emerging inflationary pressures.

\section{The CBS will require a capital injection to preserve its ability to implement} prudent monetary policies. Significant valuation losses, due to the appreciation of the rupee, have eroded its capital. The government will replenish the CBS' capital base through a direct securitized injection by mid-2010, following the audited results of 2009, with the intention of reaching full recapitalization (MEFP, 947) estimated to amount to 2 percent of GDP. ${ }^{3}$ This would strengthen the balance sheet of the CBS and enhance its portfolio of tradable securities to be used in open market operations.

20. The exchange regime will continue to be a float, which has served Seychelles well to date and helped ease the burden on monetary policy. The flexible regime will continue to provide an important cushion against external shocks. The authorities are committed to a policy of intervening in the foreign exchange market to smooth fluctuations and ensure orderly market operation, subject to achieving the reserve money and external reserves targets.

21. The strengthening of the banking sector will be essential to underpin long-term stability of the financial system. While the system remains adequately capitalized, the removal of financial repression policies created room for private credit growth, which places additional emphasis on prudential supervision (Table 6). The CBS will implement its 18-month action plan for strengthening risk based supervision, increase the minimum capital requirements for banks and will promote the use of the net tangible capital ratio as an added measure for capital adequacy. From end-2009 the government will also transfer the supervision of nonbank financial institutions to the CBS to allow for more effective and unified oversight of bank and nonbank institutions.

22. Money market development and reinforced liquidity management are important priorities. The primary market for T-bills is still shallow and there is virtually no secondary trading, partly because the infrastructure is not yet developed, but also because weaknesses in liquidity management. To facilitate market making, the authorities intend to introduce a primary dealer system.

23. The program includes steps to consolidate and divest publicly owned financial institutions. The government wholly owns the Savings Bank and a majority of Nouvobanq; a contract to prepare them for privatization will be tendered internationally by end-2009. The objective is to attract strategic banking investors, to bring in fresh capital, expertise, and stimulate competition. Three nondeposit taking institutions - the Development Bank of Seychelles, Housing Finance Corporation, and Concessional Credit Agency will also be assessed with a view to developing an exit strategy including consolidation and possible privatization, with the help of FIRST initiative.

\footnotetext{
${ }^{3}$ The issuance of treasury securities in exchange for an increase in the government's equity in the central bank is recorded in financing. Interest on the securities is in expenditure.
} 


\section{Structural Reform}

24. The major objective of the EFF is to launch second generation reforms and address long standing structural imbalances (MEFP, $\{30-32 ; 36)$. Some of the key reforms - public enterprise restructuring, tax reform, PFM - have already been initiated under the SBA, but more sustained efforts will be needed going forward. The authorities' medium-term reform strategy is intended to provide a comprehensive policy framework in all major areas to serve a coordinating function for donors and stakeholders. A public sector strategic assessment and action plan will be finalized in discussion with World Bank staff.

25. In a number of sectoral areas reforms are needed to raise economic performance and address potentially macroeconomically important risks (MEFP, |26-27). The authorities are eager to move in these areas and the mission advised them to obtain relevant technical support from the World Bank and others. These include the promotion of a better business climate; more flexible and efficient labor laws; environmental policies, and sectoral policies for air access, tourism, and fishing. Pension reform also appears necessary as the funding is inadequate; tied to labor law reform, pension reform could further improve the incentive to work. Greater transparency in data publication on fishing licenses and agreements will support better natural resource management. National statistics are being reinforced and the trade regime reformed in compliance with Seychelles' commitments to COMESA and reactivated application to the WTO.

\section{Program Financing, Design, Monitoring, ANd Risks}

\section{A. Financing}

26. The program financing gaps for 2010-12 are expected to be closed by the restructuring of public external debt and disbursement of resources available under the proposed EFF arrangement (Table 7, MEFP, ๆ49).

\section{B. Program Design and Monitoring}

27. The program sets quarterly quantitative performance criteria on net international reserves (NIR), reserve money, and the fiscal primary balances (MEFP, $\mid 50-52$ ). There would be semi-annual program reviews with quarterly disbursements. The first program review under the EFF would be based on end-March 2010 performance. Financing assurances reviews would be needed until arrears to private creditors are cleared. Quarterly structural benchmarks would mark progress on the structural reform agenda, which will focus on PFM and public and financial sector reform.

28. Access under the three-year EFF is proposed at 225 percent of quota (SDR 19.8 million) with 10 percent of quota disbursed upon approval of the arrangement (Table 8). This level of access is needed to close residual financing gaps, after assumed debt restructuring, and support the continued rebuilding of external reserves from depleted levels. Thereafter, the arrangement would be phased in the first year to mirror access under the Stand-By Arrangement (85 percent of quota); with annual access declining 
to 80 and 60 percent in the second and third years of the arrangement, respectively. Under the proposed access levels, Seychelles should not have any difficulty meeting its obligations to the Fund as indicators of outstanding credits and servicing ratios remain moderate (Table 9).

\section{Risks}

29. The main risk to the outlook stems from uncertainty about the depth and duration of the global recession. Apart from global uncertainty and piracy in the Indian Ocean affecting the outlook for tourism and FDI inflows, the main sources of risks to the program stem from possible significant delays in the public external debt restructuring, capacity constraints on reform implementation, and resistance to reform by vested interests. Good progress in discussions with creditors and the imminent launching of a debt exchange offer to commercial bondholders give comfort that a debt restructuring on appropriate terms is within reach. When set against the authorities' very strong ownership of the program, broad public and political support for the reforms, and track record under the SBA, these risks appear manageable.

\section{Staff Appraisal}

30. Staff commends the Seychelles authorities for the remarkable progress achieved over the past 12 months in addressing long standing economic problems. In the space of a few months inflation has been stopped and a degree of macroeconomic stability established following a period of volatility. Program implementation has been exemplary, even in the face of strong head winds from the global recession. Strong ownership and broad public support for the reforms have been the keys to the program's success. The evident success of the macroeconomic and financial reforms is leveraging further progress on governance and transparency. Progress has also been made in negotiations with external creditors aimed at achieving a restructuring compatible with Seychelles's payments capacity. Given their continuing good faith efforts to reach agreement on comparable terms with all creditors, as evidenced by the ongoing dialogue and timely sharing of information, the staff believes the authorities are in compliance with the Fund's lending into arrears policy.

\section{The very strong fiscal adjustment in $\mathbf{2 0 0 9}$ has played the key role in stabilizing} the economy. Staff welcomes the good progress made on PFM, and the 2010 budget represents another major step forward in improving the transparency and performance of the central government. The introduction of a treasury single account has strengthened control over public finance. The authorities are encouraged to maintain the pace of these efforts that are central to achieving fiscal sustainability and creating the space for much needed public investment. The 2010 budget will also introduce the first step of a major overhaul of the tax regime to reduce distortions and improve the business climate.

32. The new market based monetary policy is working well and made an important contribution to the stabilization. Looking ahead, efforts should focus on further expanding monetary policy instruments, modernizing financial infrastructure, and strengthening the financial sector. Some volatility in interest and exchange rate movements in October reflects teething problems in treasury management which has been strengthened for more consistency going forward. The managed floating exchange regime has played a key role in the adjustment effort and remains appropriate. 
33. In light of the strong performance through end-September, compliance with the performance criteria and consistency with the Fund's LIA policy, staff recommends completion of the third Stand-By Arrangement program and financing assurances reviews.

34. Staff welcomes the authorities' request for a three-year EFF in support of their medium-term reform agenda. The SBA-supported program successfully addressed the foreign exchange distortions, implemented a large fiscal adjustment, and instituted a marketbased monetary policy, while taking first steps toward much-needed structural reform. With these objectives achieved, the focus now shifts to implementation of a second generation of reforms in a medium-term context. The key objectives in the medium term are to consolidate macroeconomic stability, remove constraints to growth, and improve the performance of the public sector. Public financial management must be strengthened, governance standards raised in the public enterprises, taxation reformed, the financial system bolstered, and the business environment improved.

35. Staff encourages the authorities to press ahead with efforts to reinforce financial discipline and accountability in the parastatal sector. Major improvements in public sector governance practices are needed over the medium term to contain fiscal risks and raise productivity of the parastatal sector. Privatization, closings, liquidation, and consolidations are options for the various entities of the sector.

36. Staff encourages the authorities to continue to reinforce their debt management capacity and to pursue their public external debt restructuring negotiations. Continued Fund involvement is crucial to the success of the reform and debt restructuring effort.

37. Staff believes that the policies in place are sufficient to achieve the program's objectives. The program is financed, assuming a public debt restructuring consistent with Seychelles' payments capacity is achieved. The good progress made in debt restructuring discussions is reassuring. The risks to the program appear manageable, especially in light of the authorities' evident commitment to, and broad public support for, the reforms. Staff recommends approval of the authorities' request for a three year arrangement under the EFF in replacement of the remainder of the SBA. 
Table 1. Seychelles: Selected Economic and Financial Indicators, 2007-12

\begin{tabular}{|c|c|c|c|c|c|c|c|}
\hline & \multirow[t]{2}{*}{2007} & \multirow[t]{2}{*}{2008} & \multicolumn{2}{|c|}{2009} & \multirow{2}{*}{2010} & \multirow{2}{*}{$\frac{2011}{\text { Projection }}$} & \multirow[t]{2}{*}{2012} \\
\hline & & & Program & Projection & & & \\
\hline National income and prices ${ }^{1}$ & \multicolumn{7}{|c|}{ (Percentage change, unless otherwise indicated) } \\
\hline Nominal GDP (millions of Seychelles rupees) & 6,877 & 8,756 & 10,280 & 10,402 & 11,057 & 11,891 & 12,872 \\
\hline Real GDP & 9.7 & -0.9 & -10.7 & -7.6 & 4.0 & 5.0 & 5.0 \\
\hline $\mathrm{CPI}$ (annual average) & 5.3 & 37.0 & 35.1 & 32.9 & 2.3 & 2.5 & 3.2 \\
\hline $\mathrm{CPI}$ (end-of-period) & 16.8 & 63.3 & 5.4 & 1.9 & 2.2 & 3.0 & 3.0 \\
\hline GDP deflator average & 11.4 & 28.4 & 31.4 & 28.5 & 2.2 & 2.4 & 3.1 \\
\hline Money and credit & \multicolumn{7}{|c|}{ (Percentage change, unless otherwise indicated) } \\
\hline Net claims on private sector & 9.3 & 58.4 & 24.3 & -12.1 & 27.3 & $\ldots$ & $\ldots$ \\
\hline Broad money $(\mathrm{M} 3(\mathrm{p}))^{2}$ & -7.6 & 27.2 & 5.6 & -0.7 & 25.6 & $\ldots$ & $\ldots$ \\
\hline Reserve money & -23.1 & 0.6 & 20.6 & 32.2 & 18.4 & $\ldots$ & $\ldots$ \\
\hline Velocity (GDP/ M3) & 1.5 & 1.5 & 1.6 & 1.8 & 1.5 & $\ldots$ & $\ldots$ \\
\hline Money multiplier (M3/reserve money) & 4.2 & 5.3 & 4.7 & 4.0 & 4.2 & $\ldots$ & $\ldots$ \\
\hline Savings-Investment balance & \multicolumn{7}{|c|}{ (In percent of GDP) } \\
\hline External savings & 20.8 & 44.7 & 29.7 & 22.6 & 32.7 & 30.1 & 28.8 \\
\hline Gross national savings & 8.0 & -12.7 & -1.8 & 6.8 & -0.9 & 2.5 & 4.7 \\
\hline Of which: government savings & -4.4 & 4.2 & 1.0 & 2.8 & 2.7 & 5.6 & 7.8 \\
\hline Gross investment & 28.8 & 32.1 & 27.9 & 29.4 & 31.8 & 32.6 & 33.6 \\
\hline Of which: government investment & 5.5 & 2.1 & 4.3 & 5.3 & 6.8 & 7.4 & 8.6 \\
\hline \multicolumn{8}{|l|}{ Government budget } \\
\hline Total revenue, excluding grants & 32.0 & 32.8 & 35.1 & 35.6 & 30.7 & 31.5 & 31.3 \\
\hline Expenditure and net lending & 40.9 & 39.8 & 35.6 & 35.1 & 32.8 & 33.4 & 32.1 \\
\hline Current expenditure & 36.3 & 28.6 & 34.2 & 32.8 & 28.0 & 25.9 & 23.5 \\
\hline Capital expenditure and net lending ${ }^{3}$ & 4.6 & 11.2 & 0.5 & 2.3 & 4.9 & 7.4 & 8.6 \\
\hline Overall balance, including grants & -8.7 & -3.3 & 0.0 & 2.8 & 0.9 & 0.4 & 1.4 \\
\hline Primary balance & -2.0 & 3.8 & 11.4 & 13.3 & 7.0 & 6.0 & 4.7 \\
\hline Total public debt ${ }^{4,5}$ & 129.8 & 135.6 & 149.7 & 140.1 & 109.5 & $\ldots$ & $\ldots$ \\
\hline Domestic & 65.8 & 52.3 & 41.8 & 41.6 & 37.4 & $\cdots$ & $\ldots$ \\
\hline External ${ }^{4,5}$ & 63.9 & 83.3 & 108.0 & 98.5 & 72.2 & $\cdots$ & $\ldots$ \\
\hline External sector & \multicolumn{7}{|c|}{ (In percent of GDP, unless otherwise indicated) } \\
\hline Current account balance including official transfers & -20.8 & -44.7 & -29.7 & -22.6 & -32.7 & -30.1 & -28.8 \\
\hline Total stock of arrears (millions of U.S. dollars) ${ }^{4,5}$ & 160.4 & 321.7 & 195.1 & 239.2 & $\ldots$ & $\ldots$ & $\ldots$ \\
\hline Total external debt outstanding (millions of U.S. dollars) ${ }^{4,5}$ & 710.2 & 767.1 & 732.6 & 754.7 & $708.1 \ldots$ & & $\cdots$ \\
\hline (in percent of GDP) ${ }^{4,5}$ & 69.2 & 83.3 & 108.0 & 98.5 & 72.2 & $\ldots$ & $\ldots$ \\
\hline Terms of trade ( $=$ - deterioration) & -1.2 & 0.7 & -2.1 & -2.1 & $\ldots$ & $\ldots$ & $\ldots$ \\
\hline Real effective exchange rate (end-of-period, percent change) ${ }^{\circ}$ & -25.4 & -18.4 & $\ldots$ & 13.0 & $\ldots$ & $\ldots$ & $\ldots$ \\
\hline Gross official reserves (end of year, millions of US dollars) & 10 & 51 & 108 & 153 & 202 & 252 & 302 \\
\hline In months of imports, c.i.f. & 0.1 & 0.6 & 1.3 & 1.5 & 1.9 & 2.2 & 2.5 \\
\hline \multicolumn{8}{|l|}{ Exchange rate ${ }^{\circ}$} \\
\hline Seychelles rupees per US\$1 (end of period) & 8.0 & 16.6 & $\ldots$ & 10.6 & $\ldots$ & $\ldots$ & $\ldots$ \\
\hline Seychelles rupees per US\$1 (period average) & 6.7 & 9.5 & $\ldots$ & 14.1 & $\ldots$ & $\ldots$ & $\ldots$ \\
\hline
\end{tabular}

Sources: Central Bank of Seychelles; Ministry of Finance; and IMF staff estimates and projections.

${ }^{1}$ Historic GDP data have been revised upwards in April 2009.

${ }^{2}$ In 2007 and earlier, includes domestic currency balances earmarked for pending import requests ("pipeline").

${ }^{3}$ In 2010 includes contingency spending of 1.1 percent of GDP

${ }^{4}$ Assumes April 2009 Paris Club agreement is implemented; does not include the impact of any further restructuring of public external debt.

${ }^{5}$ Includes arrears and the external debt of the central bank.

${ }^{6}$ Nominal exhange rates shown for 2009 are actual rates as of end-October 2009; REER is change from Dec. 2008 through Sep. 2009. 
Table 2. Seychelles: Balance of Payments, 2008-10

(Millions of U.S. dollars)

\begin{tabular}{|c|c|c|c|c|c|c|c|c|c|c|c|c|c|c|}
\hline & \multirow{3}{*}{$\begin{array}{c}2008 \\
\text { Prel. }\end{array}$} & \multicolumn{8}{|c|}{2009} & \multicolumn{5}{|c|}{2010} \\
\hline & & \multirow{2}{*}{$\begin{array}{r}\text { Q1 } \\
\text { Prel. }\end{array}$} & \multirow{2}{*}{$\begin{array}{c}\text { Q2 } \\
\text { Prel. }\end{array}$} & \multicolumn{2}{|c|}{ Q3 } & \multicolumn{2}{|c|}{ Q4 } & Yea & & Q1 & Q2 & Q3 & Q4 & Year \\
\hline & & & & Prog. & Proj. & Prog. & Proj. & Prog. & Proj. & Proj. & Proj. & Proj. & Proj. & Proj. \\
\hline $\begin{array}{l}\text { Current account } \\
\text { (Percent of GDP) }\end{array}$ & $\begin{array}{r}-412 \\
-45\end{array}$ & $\begin{array}{r}-16 \\
-2\end{array}$ & $\begin{array}{r}-38 \\
-5\end{array}$ & $\begin{array}{r}-41 \\
-6\end{array}$ & $\begin{array}{r}-11 \\
-1\end{array}$ & $\begin{array}{l}-72 \\
-11\end{array}$ & $\begin{array}{r}-107 \\
-14\end{array}$ & $\begin{array}{r}-202 \\
-30\end{array}$ & $\begin{array}{r}-172 \\
-22\end{array}$ & $\begin{array}{r}-56 \\
-6\end{array}$ & $\begin{array}{r}-51 \\
-5\end{array}$ & $\begin{array}{r}-40 \\
-4\end{array}$ & $\begin{array}{r}-173 \\
-18\end{array}$ & $\begin{array}{r}-321 \\
-33\end{array}$ \\
\hline Trade balance & -379 & -14 & -23 & -26 & 7 & -38 & -100 & -146 & -130 & -52 & -31 & -27 & -152 & -263 \\
\hline Exports of goods & 502 & 105 & 96 & 89 & 119 & 91 & 109 & 366 & 428 & 129 & 119 & 149 & 143 & 540 \\
\hline Of which: oil re-exports & 271 & 44 & 41 & 59 & 45 & 41 & 45 & 186 & 174 & 73 & 68 & 75 & 76 & 292 \\
\hline Of which: tuna exports & 215 & 50 & 38 & 26 & 57 & 51 & 53 & 171 & 198 & 47 & 37 & 61 & 58 & 203 \\
\hline Imports of goods & $-1,018$ & -171 & -151 & -159 & -155 & -151 & -231 & -608 & -708 & -213 & -178 & -190 & -297 & -878 \\
\hline Oil imports & -391 & -54 & -46 & -72 & -63 & -56 & -64 & -207 & -228 & -88 & -78 & -83 & -84 & -333 \\
\hline FDI-related imports & -275 & -38 & -41 & -22 & -35 & -45 & -40 & -146 & -153 & -41 & -45 & -49 & -68 & -204 \\
\hline Other & -352 & -80 & -64 & -66 & -57 & -50 & -126 & -255 & -327 & -83 & -54 & -59 & -144 & -341 \\
\hline Exports of services & 517 & 118 & 99 & 110 & 124 & 94 & 125 & 372 & 466 & 112 & 109 & 120 & 121 & 462 \\
\hline Of which: tourism earnings & 270 & 59 & 46 & 66 & 69 & 50 & 64 & 209 & 238 & 61 & 56 & 64 & 69 & 250 \\
\hline Imports of services & -379 & -65 & -66 & -66 & -81 & -72 & -104 & -276 & -316 & -81 & -81 & -106 & -120 & -387 \\
\hline Income, net & -103 & -19 & -31 & -28 & -27 & -47 & -32 & -107 & -109 & -18 & -30 & -25 & -33 & -106 \\
\hline Of which: transfers of profits and dividends & -61 & -16 & -16 & -23 & -21 & -29 & -20 & -64 & -73 & -15 & -15 & -20 & -17 & -67 \\
\hline Of which: interest payments due ${ }^{1}$ & -33 & -3 & -16 & -3 & -2 & -14 & -15 & -36 & -36 & -2 & -14 & -2 & -15 & -32 \\
\hline Moratorium interest & 0 & 0 & -4 & 0 & 0 & -2 & -2 & -4 & -5 & 0 & -2 & 0 & -1 & -3 \\
\hline Other interest (scheduled) & -33 & -3 & -13 & -3 & -2 & -13 & -13 & -31 & -31 & -2 & -12 & -2 & -13 & -29 \\
\hline Current transfers, net & 70 & 17 & 16 & 13 & 8 & 12 & 26 & 52 & 67 & 15 & 10 & 12 & 12 & 48 \\
\hline General government, net & 45 & 12 & 8 & 5 & 0 & 5 & 15 & 27 & 35 & 10 & 2 & 4 & 1 & 17 \\
\hline Other sectors, net & 25 & 5 & 8 & 8 & 8 & 7 & 11 & 25 & 31 & 5 & 8 & 8 & 11 & 31 \\
\hline Capital and financial account & 119 & 45 & -7 & 38 & 25 & 61 & 92 & 147 & 155 & 58 & 30 & 57 & 144 & 291 \\
\hline Capital account & 5 & 0 & 7 & 37 & 40 & 0 & 6 & 37 & 53 & 4 & 4 & 46 & 8 & 62 \\
\hline Financial account & 113 & 45 & -14 & 1 & -15 & 61 & 86 & 110 & 102 & 54 & 26 & 11 & 136 & 229 \\
\hline Direct investment, net & 350 & 47 & 51 & 27 & 44 & 56 & 50 & 183 & 192 & 50 & 54 & 57 & 84 & 245 \\
\hline Portfolio investment, net & 1 & 0 & 0 & 0 & 0 & 0 & 0 & 0 & 1 & 0 & 0 & 0 & 0 & 1 \\
\hline Other investment, net & -238 & -3 & -65 & -26 & -59 & 4 & 36 & -73 & -90 & 4 & -29 & -46 & 52 & -17 \\
\hline Assets & -3 & -10 & -32 & 2 & -7 & 13 & 17 & -10 & -32 & 12 & -5 & -10 & 19 & 16 \\
\hline Liabilities & -235 & 7 & -33 & -28 & -52 & -8 & 19 & -63 & -58 & -8 & -24 & -36 & 33 & -33 \\
\hline Government and government-guaranteed & -105 & -14 & -33 & -28 & -51 & -8 & 8 & -85 & -89 & -12 & -14 & -42 & 11 & -55 \\
\hline Disbursements & 84 & 1 & 0 & 24 & 0 & 5 & 24 & 29 & 25 & 1 & 1 & 9 & 24 & 36 \\
\hline Project loans & 84 & 1 & 0 & 5 & 0 & 5 & 5 & 10 & 5 & 1 & 1 & 9 & 4 & 16 \\
\hline Program loans & 0 & 0 & 0 & 19 & 0 & 0 & 20 & 19 & 20 & 0 & 0 & 0 & 20 & 20 \\
\hline Amortization (Principal payments) ${ }^{1,2}$ & -189 & -15 & -35 & -15 & -14 & -15 & -15 & -81 & -80 & -13 & -16 & -13 & -13 & -52 \\
\hline Deferred interest 1,2 & & 0 & 3 & 0 & 0 & 2 & 2 & 3 & 4 & 0 & 1 & 0 & 1 & 1 \\
\hline Debt forgiveness & & 0 & 0 & -37 & -36 & 0 & -3 & -37 & -36 & 0 & 0 & -39 & 0 & -39 \\
\hline Parastatals & 0 & -1 & 0 & 0 & -1 & 0 & 0 & 0 & -2 & -1 & 0 & -1 & 0 & -2 \\
\hline Disbursements & 0 & 0 & 0 & 0 & 0 & 0 & 0 & 0 & 0 & 0 & 0 & 0 & 0 & 0 \\
\hline Amortization & 0 & -1 & 0 & 0 & -1 & 0 & 0 & 0 & -2 & -1 & 0 & -1 & 0 & -2 \\
\hline Other & -130 & 22 & 0 & 0 & 0 & 0 & 11 & 22 & 33 & 5 & -10 & 7 & 22 & 24 \\
\hline of which: SDR Allocation (- = increase) ${ }^{3}$ & & & & & -11 & & -1 & & -12 & & & & & \\
\hline Net errors and omissions & 82 & 2 & 27 & 0 & 11 & 0 & 0 & 23 & 40 & 0 & 0 & 0 & 0 & 0 \\
\hline Overall balance & -212 & 31 & -18 & -3 & 24 & -11 & -15 & -32 & 23 & 2 & -22 & 17 & -29 & -30 \\
\hline Financing & 212 & -31 & 18 & -14 & -24 & -9 & -14 & -49 & -52 & -1 & 5 & -14 & -29 & -39 \\
\hline Change in net international reserves (increase: -) & 38 & -34 & -8 & -14 & -39 & -9 & -14 & -54 & -95 & -1 & 5 & -14 & -29 & -39 \\
\hline Change in gross official reserves (increase: -) & 28 & -34 & -9 & -15 & -40 & -11 & -19 & -58 & -102 & -5 & 2 & -18 & -29 & -49 \\
\hline Prospective liabilities to IMF, net & 10 & 0 & 1 & 1 & 1 & 1 & 5 & 4 & 8 & 3 & 3 & 3 & 0 & 10 \\
\hline Other NFA (increase: -) & 0 & -10 & -1 & 0 & 0 & 0 & 0 & -10 & -12 & 0 & 0 & 0 & 0 & 0 \\
\hline Exceptional financing & 174 & 13 & 27 & 0 & 15 & 0 & 0 & 15 & 55 & 0 & 0 & 0 & 0 & 0 \\
\hline Change in arrears (increase: + ) 4,5 & 174 & 11 & 26 & 0 & -126 & 0 & -9 & -125 & -97 & 0 & 0 & 0 & 0 & 0 \\
\hline Clearance of arrears & $\ldots$ & 2 & 0 & 0 & 141 & 0 & 9 & 140 & 152 & 0 & 0 & 0 & 0 & 0 \\
\hline Financing gap & 0 & 0 & 0 & 17 & 0 & 21 & 29 & 80 & 29 & 0 & 16 & -2 & 57 & 69 \\
\hline Memorandum items: & & & & & & & & & & & & & & \\
\hline Exports growth, percent & 28 & -11 & -21 & -37 & -16 & -21 & -10 & -26 & -15 & 24 & 24 & 26 & 31 & 26 \\
\hline Tourism growth, percent & -5 & -28 & -35 & 4 & 8 & 0 & 22 & -22 & -12 & 3 & 21 & -7 & 7 & 5 \\
\hline Imports growth, percent & 39 & -24 & -33 & -35 & -37 & -53 & -29 & -40 & -31 & 25 & 18 & 23 & 29 & 24 \\
\hline Exports, percent of GDP & 54 & $\ldots$ & $\ldots$ & $\ldots$ & $\ldots$ & $\ldots$ & $\ldots$ & 54 & 56 & 0 & 0 & 0 & 0 & 46 \\
\hline Imports, percent of GDP & 111 & $\ldots$ & $\ldots$ & $\ldots$ & $\ldots$ & $\ldots$ & $\ldots$ & 90 & 92 & 0 & 0 & 0 & 0 & 74 \\
\hline FDI, percent of GDP & 38 & $\ldots$ & $\ldots$ & $\ldots$ & $\ldots$ & $\ldots$ & $\ldots$ & 27 & 25 & $\ldots$ & $\ldots$ & $\ldots$ & $\ldots$ & 25 \\
\hline Gross international reserves (stock, e.o.p.) ${ }^{3}$ & 51 & 85 & 94 & 98 & 135 & 108 & 153 & 108 & 153 & 158 & 156 & 174 & 202 & 202 \\
\hline Months of prospective imports of goods and services & 0.6 & 0.8 & 0.9 & 1.2 & 1.3 & 1.3 & 1.5 & 1.3 & 1.5 & 1.5 & 1.5 & 1.6 & 1.9 & 1.9 \\
\hline Scheduled public external debt service ${ }^{1,0}$ & 221 & 18 & 49 & 18 & 18 & 28 & 28 & 114 & 113 & 16 & 29 & 15 & 27 & 86 \\
\hline (Percent of exports of goods and services) & 22 & 8 & 25 & 9 & 7 & 15 & 12 & 15 & 13 & 7 & 13 & 6 & 10 & 8.5 \\
\hline Public external debt ${ }^{1,5,6}$ & 767 & $\ldots$ & $\ldots$ & $\ldots$ & $\ldots$ & $\ldots$ & $\ldots$ & 733 & 755 & $\ldots$ & $\ldots$ & $\ldots$ & $\ldots$ & 708 \\
\hline (Percent of GDP) & 83 & $\ldots$ & $\ldots$ & $\ldots$ & $\ldots$ & $\ldots$ & $\ldots$ & 108 & 99 & $\ldots$ & $\ldots$ & $\ldots$ & $\ldots$ & 72.2 \\
\hline Of which: arrears ${ }^{1,4,5}$ & 322 & $\ldots$ & $\ldots$ & $\ldots$ & $\ldots$ & $\ldots$ & $\ldots$ & 195 & 239 & $\ldots$ & $\ldots$ & $\ldots$ & $\ldots$ & 231 \\
\hline (Percent of GDP) & 35 & $\ldots$ & $\ldots$ & $\ldots$ & $\ldots$ & $\ldots$ & $\ldots$ & 29 & 31 & $\ldots$ & $\ldots$ & $\ldots$ & $\ldots$ & 23.5 \\
\hline GDP & 921 & 766 & 766 & $\ldots$ & 766 & $\ldots$ & $\ldots$ & 679 & 766 & $\ldots$ & $\ldots$ & $\ldots$ & $\ldots$ & 981 \\
\hline
\end{tabular}

${ }^{1}$ Projections are based on identified new borrowing and rescheduled Paris Club debt. It assumes comparable cash flow treatment and identical reduction in the debt stock for Malaysia and South Africa, which also signed the agreement, and no debt restructuring for other countries. Arrears restructuring leads to higher rescheduled interest payments.

${ }^{2}$ Based on Paris Club agreement, the payments of principal and most interest during 2008 and through June 2009 are deferred.

${ }^{3}$ Seychelles received an SDR allocation of SDR 7.9 million and SDR 0.4 million in August and September 2009 respectively.

${ }^{4}$ In 2008, includes accelerated promissory notes.

${ }^{5}$ Debt forgivenesss reflects: (i) the first stage of debt reduction (about 22.5) percent in July 2009; (ii) the second stage reduction (about 22.5 percent) in July 2010 on Paris Club debt. Assumes debt reduction comparable to the Paris Club agreement on Malaysian and South African debt. 
Table 3. Seychelles: Consolidated Government Operations, 2007-2012 ${ }^{1}$

\begin{tabular}{|c|c|c|c|c|c|c|c|c|c|c|c|c|c|c|c|c|}
\hline & \multirow{3}{*}{2008} & \multicolumn{8}{|c|}{2009} & \multicolumn{5}{|c|}{2010} & \multirow{2}{*}{2011} & \multirow{2}{*}{2012} \\
\hline & & \multirow{2}{*}{$\begin{array}{r}\text { Q1 } \\
\text { Act. }\end{array}$} & \multirow{2}{*}{$\begin{array}{l}\text { Q2 } \\
\text { Act. }\end{array}$} & \multicolumn{2}{|c|}{$\mathrm{Q}^{3}$} & \multicolumn{2}{|c|}{ Q4 } & \multicolumn{2}{|c|}{$\begin{array}{l}\text { Year } \\
\end{array}$} & \multirow{2}{*}{$\begin{array}{r}\text { Q1 } \\
\text { Proj. } \\
\end{array}$} & \multirow{2}{*}{$\begin{array}{r}\text { Q2 } \\
\text { Proj. } \\
\end{array}$} & \multirow{2}{*}{$\begin{array}{l}\text { Q3 } \\
\text { Proj. }\end{array}$} & Q4 & $\begin{array}{l}\text { Year } \\
\end{array}$ & & \\
\hline & & & & Prog. & Act. & Prog. & Proj. & Prog. & Proj. & & & & Proj. & Proj. & Proj. & Proj. \\
\hline Total revenue and grants & 3,190 & 947 & 958 & 876 & 1,018 & 941 & 1,015 & 3,657 & 3,938 & 835 & 844 & 1,006 & 1,039 & 3,723 & 4,008 & 4,307 \\
\hline Total revenue & 2,868 & 944 & 848 & 854 & 1,018 & 936 & 893 & 3,611 & 3,704 & 776 & 782 & 885 & 946 & 3,390 & 3,747 & 4,032 \\
\hline Tax & 2,456 & 820 & 780 & 756 & 814 & 756 & 775 & 3,066 & 3,190 & 712 & 713 & 797 & 845 & 3,067 & 3,365 & 3,632 \\
\hline $\begin{array}{l}\text { Personal Income Tax } \\
\text { Social securitytax }\end{array}$ & & 0 & 0 & & 0 & & 0 & & 0 & 0 & 0 & 79 & 118 & 197 & 465 & 504 \\
\hline $\begin{array}{l}\text { Social security tax } \\
\text { Trade tax }\end{array}$ & $\begin{array}{l}295 \\
410\end{array}$ & $\begin{array}{r}94 \\
103\end{array}$ & $\begin{array}{l}101 \\
118\end{array}$ & $\begin{array}{r}93 \\
918\end{array}$ & $\begin{array}{l}95 \\
98\end{array}$ & ${ }_{123}^{93}$ & 104 & $\begin{array}{l}365 \\
466\end{array}$ & $\begin{array}{l}393 \\
459\end{array}$ & $\begin{array}{l}109 \\
104\end{array}$ & 93 & ${ }_{123}^{28}$ & $\begin{array}{r}0 \\
158\end{array}$ & $\begin{array}{l}230 \\
491\end{array}$ & $\begin{array}{r}0 \\
543\end{array}$ & $\begin{array}{r}0 \\
597-2-30\end{array}$ \\
\hline Goods and services tax (GST) & $\begin{array}{l}410 \\
951\end{array}$ & 320 & $\begin{array}{l}118 \\
342\end{array}$ & $\begin{array}{l}118 \\
346\end{array}$ & $\begin{array}{r}98 \\
322\end{array}$ & $\begin{array}{l}127 \\
344\end{array}$ & $\begin{array}{l}\begin{array}{l}139 \\
332\end{array} \\
-\end{array}$ & $\begin{array}{r}460 \\
1.322\end{array}$ & $\begin{array}{r}459 \\
1.316\end{array}$ & $\begin{array}{l}104 \\
284\end{array}$ & $\begin{array}{l}106 \\
319\end{array}$ & $\begin{array}{l}123 \\
319\end{array}$ & $\begin{array}{l}158 \\
360\end{array}$ & $\begin{array}{r}491 \\
1.282\end{array}$ & $\begin{array}{r}543 \\
1413\end{array}$ & $\begin{array}{r}597 \\
1514\end{array}$ \\
\hline Business tax & 524 & 127 & 154 & 170 & 244 & 156 & $\begin{array}{l}332 \\
135\end{array}$ & 563 & 660 & 136 & 132 & 186 & 145 & $\begin{array}{l}1,282 \\
599\end{array}$ & $\begin{array}{c}1,443 \\
644\end{array}$ & 1,514 \\
\hline Other & 277 & 176 & 65 & 30 & 55 & 36 & 65 & 350 & 361 & 80 & 63 & 61 & 64 & 269 & $\begin{array}{l}044 \\
299\end{array}$ & ${ }_{320}^{691}-2 x-10$ \\
\hline Nontax & 412 & 124 & 68 & 98 & 204 & 180 & 118 & 545 & 514 & 64 & 69 & 89 & 101 & 323 & 382 & 400 \\
\hline Fees and charges & 145 & 44 & 38 & 37 & 51 & 40 & 37 & 178 & 169 & 44 & 44 & 44 & 44 & 174 & 205 & 210 \\
\hline Dividends from parastatals & 64 & 5 & 26 & 14 & 105 & 89 & 27 & 150 & 163 & 10 & 20 & 30 & 40 & 99 & 117 & 127 \\
\hline & 203 & 75 & & 47 & & 51 & 54 & & 181 & 10 & 6 & 15 & 18 & 50 & 59 & 64 \\
\hline External grants & 321 & 3 & 110 & 22 & 0 & 5 & 122 & 46 & 234 & 59 & 62 & 121 & 92 & 333 & 262 & 275 \\
\hline Expenditure and net lending & $\begin{array}{l}3,483 \\
3,53\end{array}$ & 511 & 1,044 & 913 & 856 & 1,129 & $\begin{array}{l}1,239 \\
1,02\end{array}$ & $\begin{array}{l}3,660 \\
3511\end{array}$ & $\begin{array}{l}3,651 \\
3408\end{array}$ & $\begin{array}{l}670 \\
655\end{array}$ & $\begin{array}{l}901 \\
839-1-20\end{array}$ & 878 & 1,179 & 3,628 & 3,966 & 4,128 \\
\hline $\begin{array}{l}\text { Current expenditure } \\
\text { Primary current expenditure }\end{array}$ & $\begin{array}{l}2,553 \\
1,875\end{array}$ & $\begin{array}{l}732 \\
490\end{array}$ & $\begin{array}{l}928 \\
525\end{array}$ & $\begin{array}{l}794 \\
609\end{array}$ & $\begin{array}{l}727 \\
503\end{array}$ & $\begin{array}{l}967 \\
612\end{array}$ & $\begin{array}{r}1,021 \\
792\end{array}$ & $\begin{array}{l}3,511 \\
2,335\end{array}$ & $\begin{array}{l}3,408 \\
2,310\end{array}$ & $\begin{array}{l}655 \\
556\end{array}$ & $\begin{array}{l}839 \\
606\end{array}$ & $\begin{array}{l}696 \\
598\end{array}$ & $\begin{array}{l}900 \\
655\end{array}$ & $\begin{array}{l}3,091 \\
2,415\end{array}$ & $\begin{array}{l}3,801 \\
2,405\end{array}$ & $\begin{array}{l}3,022 \\
2,601\end{array}$ \\
\hline Wages and salaries & 688 & 199 & 167 & 185 & 171 & 188 & 231 & 768 & 768 & 176 & 176 & 176 & 176 & 704 & $\begin{array}{l}2,4004 \\
684\end{array}$ & $\begin{array}{l}2,001 \\
741\end{array}$ \\
\hline Goods and services & 489 & 118 & 172 & 185 & 165 & 188 & 233 & 661 & 689 & 150 & 194 & 191 & 219 & 754 & 811 & 878 \\
\hline Interest payments due & 629 & 242 & 404 & 185 & 224 & 355 & 229 & 1,176 & 1,098 & 100 & 233 & 98 & 245 & 676 & 676 & 421 \\
\hline Foreign interest ${ }^{\circ}$ & & & 234 & 39 & & 212 & 159 & 541 & 484 & 24 & 156 & 19 & 169 & 367 & 379 & 148 \\
\hline Domestic interest & 285 & 182 & 170 & 146 & 192 & 143 & 70 & 635 & 614 & 75 & 77 & 79 & 77 & 309 & 297 & 273 \\
\hline Transfers & 692 & 166 & 175 & 238 & 166 & 235 & 327 & 886 & 833 & 227 & 233 & 228 & 257 & 945 & 898 & 970 \\
\hline Social program of central government & 192 & 40 & 62 & 87 & 64 & 84 & 136 & 285 & 302 & 75 & 82 & 77 & 105 & 339 & 312 & 336 \\
\hline Transfers to public sector from central government & 220 & 47 & 43 & 60 & 23 & 59 & 92 & 246 & 205 & 64 & 64 & 64 & 64 & 255 & 246 & 267 \\
\hline Benefits and programs of Social Security Fund & 279 & 79 & 70 & 91 & 79 & 92 & 99 & 355 & 326 & 88 & 88 & 88 & 88 & 351 & 340 & 368 \\
\hline Other & 6 & 7 & 10 & 2 & 1 & 1 & 2 & 21 & 20 & 3 & 3 & 3 & 3 & 12 & 12 & 12 \\
\hline Capital expenditure & 183 & 21 & 159 & 136 & 135 & 177 & 240 & 446 & 556 & 104 & 137 & 237 & 269 & 747 & 885 & 1,106 \\
\hline $\begin{array}{l}\text { Net lending } \\
\text { Contingency }\end{array}$ & 796 & -242 & $\begin{array}{r}-43 \\
0\end{array}$ & $\begin{array}{l}-68 \\
-60\end{array}$ & $\begin{array}{c}-9 \\
3\end{array}$ & $\begin{array}{l}-65 \\
50\end{array}$ & $\begin{array}{r}-21 \\
0\end{array}$ & -397 & $\begin{array}{r}-315 \\
3\end{array}$ & $\begin{array}{r}-90 \\
0\end{array}$ & $\begin{array}{r}-100 \\
25\end{array}$ & $\begin{array}{r}-100 \\
45\end{array}$ & $\begin{array}{r}-40 \\
50\end{array}$ & $\begin{array}{c}-330 \\
120\end{array}$ & $\begin{array}{l}0 \\
0\end{array}$ & $\begin{array}{l}0 \\
0\end{array}$ \\
\hline Primary balance ${ }^{c}$ & 336 & 678 & 317 & 148 & 386 & 167 & 4 & 1,173 & 1,385 & 265 & 176 & 226 & 105 & 771 & 717 & 600 \\
\hline Overall balance, commitment basis & -293 & 436 & -87 & -37 & 162 & -188 & -224 & -3 & 287 & 165 & -57 & 128 & -140 & 95 & 42 & 179 \\
\hline Change in arrears & 31 & -44 & 105 & -50 & -59 & -34 & -44 & -148 & -42 & -25 & -25 & -25 & -25 & -100 & 0 & 0 \\
\hline External interest & 167 & 16 & 155 & 0 & 12 & 0 & 0 & 47 & 184 & 0 & 0 & 0 & 0 & 0 & 0 & 0 \\
\hline & -136 & -61 & -50 & -50 & -71 & -34 & -44 & -195 & -226 & -25 & -25 & -25 & -25 & -100 & 0 & 0 \\
\hline Overall balance, cash basis (after grants) & -262 & 392 & 18 & -87 & 103 & -222 & -268 & -152 & 244 & 140 & -82 & 103 & -165 & -5 & 42 & 179 \\
\hline Financing & 262 & -392 & -18 & 87 & -103 & 222 & 268 & 152 & -244 & -140 & 82 & -103 & 165 & 5 & -42 & -179 \\
\hline Foreign financing & 233 & -18 & -242 & 127 & & -175 & 116 & -852 & -132 & -132 & -156 & -36 & 129 & -194 & $-2,619$ & 336 \\
\hline Disbursements & 672 & 12 & 2 & 352 & 0 & 73 & 260 & 427 & 274 & 11 & 17 & 107 & 270 & 405 & 424 & 482 \\
\hline Project loans & 672 & 12 & 2 & 73 & 0 & 73 & 49 & 149 & 62 & 11 & 17 & 107 & 46 & 181 & 188 & 241 \\
\hline Program/budget support & & 0 & $\begin{array}{r}0 \\
-50\end{array}$ & 278 & 0 & 0 & 211 & 278 & 211 & 0 & 0 & 0 & 224 & 224 & 236 & 241 \\
\hline 'Scheduled amortiz & $-1,630$ & -228 & -502 & -224 & -170 & -248 & -163 & $-1,279$ & $-1,063$ & -143 & -179 & -143 & -146 & -611 & $-3,055$ & -159 \\
\hline arrears & 1,191 & 159 & 220 & 0 & $-1,611$ & 0 & -93 & $-1,827$ & $-1,325$ & 0 & 0 & 0 & 0 & 0 & 0 & 0 \\
\hline $\begin{array}{l}\text { Clearance of arrears } \\
\text { Rebtsenice relief }\end{array}$ & 0 & 40 & $\begin{array}{r}1 \\
37\end{array}$ & 0 & 1,792 & 0 & 93 & 2,055 & $\begin{array}{r}1,926 \\
57\end{array}$ & 0 & 0 & 0 & 0 & 0 & 0 & 0 \\
\hline $\begin{array}{l}\text { Debt service relief } \\
\text {. }\end{array}$ & $\begin{array}{r}0 \\
-70\end{array}$ & $\begin{array}{r}0 \\
-551\end{array}$ & $\begin{array}{r}37 \\
330\end{array}$ & $\begin{array}{r}0 \\
-379\end{array}$ & $\begin{array}{r}0 \\
-170\end{array}$ & 0 & $\begin{array}{r}20 \\
-17\end{array}$ & $\begin{array}{r}0 \\
-576\end{array}$ & $\begin{array}{r}57 \\
-508\end{array}$ & $\begin{array}{r}0 \\
-15\end{array}$ & 6 & $\begin{array}{r}0 \\
-65\end{array}$ & $\begin{array}{r}6 \\
-53 \\
-53\end{array}$ & $\begin{array}{r}12 \\
-517\end{array}$ & $\begin{array}{r}12 \\
-45\end{array}$ & $\begin{array}{r}13 \\
-771 \\
\end{array}$ \\
\hline $\begin{array}{l}\text { Domestit rinaning, net } \\
\text { Bank financing }\end{array}$ & $\begin{array}{c}-100 \\
-100-x y\end{array}$ & $\begin{array}{r}-551 \\
-1,396\end{array}$ & $\begin{array}{l}330 \\
773\end{array}$ & $\begin{array}{l}-399 \\
-360\end{array}$ & $\begin{array}{r}-170 \\
-348\end{array}$ & $\begin{array}{l}4 \\
4\end{array}$ & $\begin{array}{l}-177 \\
-386\end{array}$ & $\begin{array}{r}-576 \\
-1,508\end{array}$ & $\begin{array}{r}-508 \\
-1.356\end{array}$ & $\begin{array}{l}-15 \\
-13\end{array}$ & $\begin{array}{r}100 \\
90\end{array}$ & $\begin{array}{l}-65 \\
-58\end{array}$ & $\begin{array}{l}-537 \\
-484\end{array}$ & $\begin{array}{l}-517 \\
-465\end{array}$ & $\begin{array}{l}-455 \\
-432\end{array}$ & $\begin{array}{l}-771 \\
-732\end{array}$ \\
\hline CBS & -3 & $-1,015$ & 415 & -38 & -375 & 0 & -41 & -967 & $-1,015$ & -4 & 230 & -19 & -161 & 45 & -45 & -77 \\
\hline CBS recapitalization & & & & & & & & & & & -200 & & & -200 & & \\
\hline Commercial banks & -96 & -380 & 357 & -322 & 27 & 4 & -345 & -54 & -341 & -9 & 60 & -39 & -322 & -310 & -386 & -655 \\
\hline Nonbank & 30 & 845 & -442 & -19 & 177 & 0 & -20 & 932 & 559 & -1 & 10 & -6 & -54 & -52 & -23 & -39 \\
\hline Privatization and long-term lease of fixed assets & 219 & 97 & 61 & 90 & 26 & 90 & 9 & 370 & 193 & 50 & 55 & 65 & 80 & 250 & 269 & 256 \\
\hline Statistical discrepancy & -120 & 80 & -168 & 0 & 30 & 0 & 0 & 0 & -57 & 0 & 0 & 0 & 0 & 0 & 0 & 0 \\
\hline Fiscal financing gap & 0 & 0 & 0 & 174 & 0 & 303 & 259 & 1,211 & 259 & -44 & 84 & -68 & 493 & 466 & 2,763 & 0 \\
\hline & & & & & & & & & & & & & & & & \\
\hline External debt service & 1,974 & 288 & 735 & 263 & 201 & 460 & 322 & 1,821 & 1,547 & 167 & 335 & 162 & 315 & 978 & 3,434 & 307 \\
\hline
\end{tabular}


Table 3. Seychelles: Consolidated Government Operations, $2007-2012{ }^{1}$ (continued) (Percent of GDP)

\begin{tabular}{|c|c|c|c|c|c|c|c|c|c|c|c|c|c|c|c|c|}
\hline & \multirow[t]{3}{*}{2008} & \multicolumn{8}{|c|}{2009} & \multicolumn{5}{|c|}{2010} & \multirow{3}{*}{$\begin{array}{r}2011 \\
\text { Proj. }\end{array}$} & \multirow{3}{*}{$\begin{array}{r}2012 \\
\text { Proj. }\end{array}$} \\
\hline & & \multirow{2}{*}{$\begin{array}{r}\text { Q1 } \\
\text { Act. }\end{array}$} & \multirow{2}{*}{$\begin{array}{r}\text { Q2 } \\
\text { Act. }\end{array}$} & \multicolumn{2}{|c|}{ Q3 } & \multicolumn{2}{|c|}{ Q4 } & \multicolumn{2}{|c|}{ Year } & \multirow{2}{*}{$\begin{array}{r}\text { Q1 } \\
\text { Proj. }\end{array}$} & Q2 & Q3 & Q4 & Year & & \\
\hline & & & & Prog. & Act. & Prog. & Proj. & Prog. & Proj. & & Proj. & Proj. & Proj. & Proj. & & \\
\hline Total revenue and grants & 36.4 & 9.2 & 9.3 & 8.5 & 9.9 & 9.2 & 9.8 & 35.6 & 38.2 & 7.6 & 7.6 & 9.1 & 9.4 & 33.7 & 33.7 & 33.5 \\
\hline Total revenue & 32.8 & 9.2 & 8.2 & 8.3 & 9.9 & 9.1 & 8.7 & 35.1 & 35.9 & 7.0 & 7.1 & 8.0 & 8.6 & 30.7 & 31.5 & 31.3 \\
\hline Tax & 28.1 & 8.0 & 7.6 & 7.4 & 7.9 & 7.4 & 7.5 & 29.8 & 30.9 & 6.4 & 6.4 & 7.2 & 7.6 & 27.7 & 28.3 & 28.2 \\
\hline Personal Income Tax & & & & & & & & & & 0.0 & 0.0 & 0.7 & 1.1 & 1.8 & 3.9 & 3.9 \\
\hline Social security tax & 3.4 & 0.9 & 1.0 & 0.9 & 0.9 & 0.9 & 1.0 & 3.6 & 3.8 & 1.0 & 0.8 & 0.3 & 0.0 & 2.1 & 0.0 & 0.0 \\
\hline Trade tax & 4.7 & 1.0 & 1.1 & 1.1 & 1.0 & 1.2 & 1.4 & 4.5 & 4.5 & 0.9 & 1.0 & 1.1 & 1.4 & 4.4 & 4.6 & 4.6 \\
\hline Goods and services tax (GST) & 10.9 & 3.1 & 3.3 & 3.4 & 3.1 & 3.3 & 3.2 & 12.9 & 12.8 & 2.6 & 2.9 & 2.9 & 3.3 & 11.6 & 11.9 & 11.8 \\
\hline Business tax & 6.0 & 1.2 & 1.5 & 1.7 & 2.4 & 1.5 & 1.3 & 5.5 & 6.4 & 1.2 & 1.2 & 1.7 & 1.3 & 5.4 & 5.4 & 5.4 \\
\hline Other & 3.2 & 1.7 & 0.6 & 0.3 & 0.5 & 0.3 & 0.6 & 3.4 & 3.5 & 0.7 & 0.6 & 0.6 & 0.6 & 2.4 & 2.5 & 2.5 \\
\hline Nontax & 4.7 & 1.2 & 0.7 & 1.0 & 2.0 & 1.8 & 1.1 & 5.3 & 5.0 & 0.6 & 0.6 & 0.8 & 0.9 & 2.9 & 3.2 & 3.1 \\
\hline Fees and charges & 1.7 & 0.4 & 0.4 & 0.4 & 0.5 & 0.4 & 0.4 & 1.7 & 1.6 & 0.4 & 0.4 & 0.4 & 0.4 & 1.6 & 1.7 & 1.6 \\
\hline Dividends from parastatals & 0.7 & 0.0 & 0.2 & 0.1 & 1.0 & 0.9 & 0.3 & 1.5 & 1.6 & 0.1 & 0.2 & 0.3 & 0.4 & 0.9 & 1.0 & 1.0 \\
\hline Other & 2.3 & 0.7 & 0.0 & 0.5 & 0.5 & 0.5 & 0.5 & 2.1 & 1.8 & 0.1 & 0.1 & 0.1 & 0.2 & 0.5 & 0.5 & 0.5 \\
\hline External grants & 3.7 & 0.0 & 1.1 & 0.2 & 0.0 & 0.0 & 1.2 & 0.4 & 2.3 & 0.5 & 0.6 & 1.1 & 0.8 & 3.0 & 2.2 & 2.1 \\
\hline Expenditure and net lending & 39.8 & 5.0 & 10.1 & 8.9 & 8.3 & 11.0 & 12.0 & 35.6 & 35.4 & 6.1 & 8.2 & 7.9 & 10.7 & 32.8 & 33.4 & 32.1 \\
\hline Current expenditure & 28.6 & 7.1 & 9.0 & 7.7 & 7.0 & 9.4 & 9.9 & 34.2 & 33.0 & 5.9 & 7.6 & 6.3 & 8.1 & 28.0 & 25.9 & 23.5 \\
\hline Primary current expenditure & 21.4 & 4.8 & 5.1 & 5.9 & 4.9 & 6.0 & 7.7 & 22.7 & 22.4 & 5.0 & 5.5 & 5.4 & 5.9 & 21.8 & 20.2 & 20.2 \\
\hline Wages and salaries & 7.9 & 1.9 & 1.6 & 1.8 & 1.7 & 1.8 & 2.2 & 7.5 & 7.4 & 1.6 & 1.6 & 1.6 & 1.6 & 6.4 & 5.8 & 5.8 \\
\hline Goods and services & 5.6 & 1.1 & 1.7 & 1.8 & 1.6 & 1.8 & 2.3 & 6.4 & 6.7 & 1.4 & 1.8 & 1.7 & 2.0 & 6.8 & 6.8 & 6.8 \\
\hline Interest payments due & 7.2 & 2.3 & 3.9 & 1.8 & 2.2 & 3.5 & 2.2 & 11.4 & 10.6 & 0.9 & 2.1 & 0.9 & 2.2 & 6.1 & 5.7 & 3.3 \\
\hline Foreign interest & 3.9 & 0.6 & 2.3 & 0.4 & 0.3 & 2.1 & 1.5 & 5.3 & 4.7 & 0.2 & 1.4 & 0.2 & 1.5 & 3.3 & 3.2 & 1.2 \\
\hline Domestic interest & 3.3 & 1.8 & 1.7 & 1.4 & 1.9 & 1.4 & 0.7 & 6.2 & 6.0 & 0.7 & 0.7 & 0.7 & 0.7 & 2.8 & 2.5 & 2.1 \\
\hline Transfers & 7.9 & 1.6 & 1.7 & 2.3 & 1.6 & 2.3 & 3.2 & 8.6 & 8.1 & 2.1 & 2.1 & 2.1 & 2.3 & 8.5 & 7.5 & 7.5 \\
\hline Social program of central government & 2.2 & 0.4 & 0.6 & 0.8 & 0.6 & 0.8 & 1.3 & 2.8 & 2.9 & 0.7 & 0.7 & 0.7 & 1.0 & 3.1 & 2.6 & 2.6 \\
\hline Transfers to public sector from central government & 2.5 & 0.5 & 0.4 & 0.6 & 0.2 & 0.6 & 0.9 & 2.4 & 2.0 & 0.6 & 0.6 & 0.6 & 0.6 & 2.3 & 2.1 & 2.1 \\
\hline Benefits a & 3.2 & 0.8 & 0.7 & 0.9 & 0.8 & 0.9 & 1.0 & 3.5 & 3.2 & 0.8 & 0.8 & 08 & 0.8 & 3.2 & 2.9 & 2.9 \\
\hline Other & 0.1 & 0.1 & 0.1 & 0.0 & 0.0 & 0.0 & 0.0 & 0.2 & 0.2 & 0.0 & 0.0 & 0.0 & 0.0 & 0.1 & 0.1 & 0.1 \\
\hline Capital expenditure & 2.1 & 0.2 & 1.5 & 1.3 & 1.3 & 1.7 & 2.3 & 4.3 & 5.4 & 0.9 & 1.2 & 2.1 & 2.4 & 6.8 & 7.4 & 8.6 \\
\hline Net lending & 9.1 & -2.4 & -0.4 & -0.7 & -0.1 & -0.6 & -0.2 & -3.9 & -3.1 & -0.8 & -0.9 & -0.9 & -0.4 & -3.0 & 0.0 & 0.0 \\
\hline Contingency & & 0.0 & 0.0 & 0.5 & 0.0 & 0.5 & 0.0 & 1.0 & 0.0 & 0.0 & 0.2 & 0.4 & 0.5 & 1.1 & 0.0 & 0.0 \\
\hline Primary balance ${ }^{2}$ & 3.8 & 6.6 & 3.1 & 1.4 & 3.7 & 1.6 & 0.0 & 11.4 & 13.4 & 2.4 & 1.6 & 2.0 & 0.9 & 7.0 & 6.0 & 4.7 \\
\hline Overall balance, commitment & -3.3 & 4.2 & -0.8 & -0.4 & 1.6 & -1.8 & -2.2 & 0.0 & 2.8 & 1.5 & -0.5 & 1.2 & -1.3 & 0.9 & 0.4 & 1.4 \\
\hline Change in arrears & 0.4 & -0.4 & 1.0 & -0.5 & -0.6 & -0.3 & -0.4 & -1.4 & -0.4 & -0.2 & -0.2 & -0.2 & -0.2 & -0.9 & 0.0 & 0.0 \\
\hline External interest & 1.9 & 0.2 & 1.5 & 0.0 & 0.1 & 0.0 & 0.0 & 0.5 & 1.8 & 0.0 & 0.0 & 0.0 & 0.0 & 0.0 & 0.0 & 0.0 \\
\hline Budget & -1.5 & -0.6 & -0.5 & -0.5 & -0.7 & -0.3 & -0.4 & -1.9 & -2.2 & -0.2 & -0.2 & -0.2 & -0.2 & -0.9 & 0.0 & 0.0 \\
\hline Overall balance, cash basis (after grants) & -3.0 & 3.8 & 0.2 & -0.8 & 1.0 & -2.2 & -2.6 & -1.5 & 2.4 & 1.3 & -0.7 & 0.9 & -1.5 & 0.0 & 0.4 & 1.4 \\
\hline Financing & 3.0 & -3.8 & -0.2 & 0.8 & -1.0 & 2.2 & 2.6 & 1.5 & -2.4 & -1.3 & 0.7 & -0.9 & 1.5 & 0.0 & -0.4 & -1.4 \\
\hline Foreign financing & 2.7 & -0.2 & -2.3 & 1.2 & 0.1 & -1.7 & 1.1 & -8.3 & -1.3 & -1.2 & -1.4 & -0.3 & 1.2 & -1.8 & -22.0 & 2.6 \\
\hline Disbursements & 7.7 & 0.1 & 0.0 & 3.4 & 0.0 & 0.7 & 2.5 & 4.2 & 2.7 & 0.1 & 0.2 & 1.0 & 2.4 & 3.7 & 3.6 & 3.7 \\
\hline Project loans & 7.7 & 0.1 & 0.0 & 0.7 & 0.0 & 0.7 & 0.5 & 1.4 & 0.6 & 0.1 & 0.2 & 1.0 & 0.4 & 1.6 & 1.6 & 1.9 \\
\hline Program/budget support & 0.0 & 0.0 & 0.0 & 2.7 & 0.0 & 0.0 & 2.0 & 2.7 & 2.0 & 0.0 & 0.0 & 0.0 & 2.0 & 2.0 & 2.0 & 1.9 \\
\hline Scheduled & -18.6 & -2.2 & -4.9 & -2.2 & -1.6 & -2.4 & -1.6 & -12.4 & -10.3 & -1.3 & -1.6 & -1.3 & -1.3 & -5.5 & -25.7 & -1.2 \\
\hline Change in amortization arrears & 13.6 & 1.5 & 2.1 & 0.0 & -15.6 & 0.0 & -0.9 & -17.8 & -12.8 & 0.0 & 0.0 & 0.0 & 0.0 & 0.0 & 0.0 & 0.0 \\
\hline Clearance of arrears & 0.0 & 0.4 & 0.0 & 0.0 & 17.4 & 0.0 & 0.9 & 20.0 & 18.7 & 0.0 & 0.0 & 0.0 & 0.0 & 0.0 & 0.0 & 0.0 \\
\hline Debt servic & 0.0 & 0.0 & 0.4 & 0.0 & 0.0 & 0.0 & 0.2 & 0.0 & 0.5 & 0.0 & 0.1 & 0.0 & 0.1 & 0.1 & 0.1 & 0.1 \\
\hline Domestic financing, net & -0.8 & -5.5 & 3.3 & -3.7 & -1.7 & 0.0 & -1.1 & -5.6 & -4.9 & -0.1 & 0.9 & -0.6 & -4.9 & -4.7 & -3.9 & -6.0 \\
\hline Bank financing & -1.1 & -14.0 & 7.7 & -3.6 & -3.5 & 0.0 & -3.7 & -15.1 & -13.1 & -0.1 & 0.8 & -0.5 & -4.4 & -4.2 & -3.8 & -5.7 \\
\hline CBS & & -10.2 & 4.2 & -0.4 & -3.8 & 0.0 & -0.4 & -9.7 & -9.8 & 0.0 & 2.1 & -0.2 & -1.5 & 0.4 & -0.4 & -0.6 \\
\hline CBS recapitalization & $\ldots$ & & & & & $\ldots$ & ... & & & & -1.8 & $\ldots$ & $\ldots$ & -1.8 & $\ldots$ & \\
\hline Commercial banks & & -3.8 & 3.6 & -3.2 & 0.3 & 0.0 & -3.3 & -5.4 & -3.3 & -0.1 & 0.5 & -0.4 & -2.9 & -2.8 & -3.4 & -5.1 \\
\hline Nonbank & 0.3 & 8.5 & -4.4 & -0.2 & 1.8 & 0.0 & -0.2 & 9.3 & 5.4 & 0.0 & 0.1 & -0.1 & -0.5 & -0.5 & -0.2 & -0.3 \\
\hline Privatization and long-term lease of fixed assets & 2.5 & 0.9 & 0.6 & 0.9 & 0.3 & 0.9 & 0.1 & 3.6 & 1.9 & 0.5 & 0.5 & 0.6 & 0.7 & 2.3 & 2.3 & 2.0 \\
\hline Statistical dis & -1.4 & 0.8 & -1.6 & 0.0 & 0.3 & 0.0 & 0.0 & 0.0 & -0.6 & 0.0 & 0.0 & 0.0 & 0.0 & 0.0 & 0.0 & 0.0 \\
\hline Fiscal financing gap & 0.0 & 0.0 & 0.0 & 1.7 & 0.0 & 2.9 & 2.5 & 11.8 & 2.5 & -0.4 & 0.8 & -0.6 & 4.5 & 4.2 & 23.4 & 0.0 \\
\hline Memorandum item: & & & & & & & & & & & & & & & & \\
\hline $\begin{array}{l}\text { Nominal GDP } \\
\text { Domestic debt (percent of GDP) }\end{array}$ & 8,756 & 10,402 & 10,402 & 10,280 & 10,402 & 10,280 & 10,402 & 10,280 & 10,402 & 11,057 & 11,057 & 11,057 & 11,057 & 11,057 & 11,891 & 12,872 \\
\hline Domestic debt (percent of GDP) & 53.6 & & & & & & & & & & & & & & & \\
\hline
\end{tabular}

Sources: Ministry of Finance; Social Security Fund; Pension Fund; Central Bank of Seychelles (CBS); and IMF staff estimates and projections.

Includes the central government and the social security system.

${ }^{2}$ The CBS transferred SR 175 million (2 2 percent of GDP) in profit to the government in March 2008. Of this amount SR 118.7 million (1.5 percent of GDP) was revaluation gains. 
Table 4. Seychelles: Monetary Survey and Central Bank Accounts, 2007-10

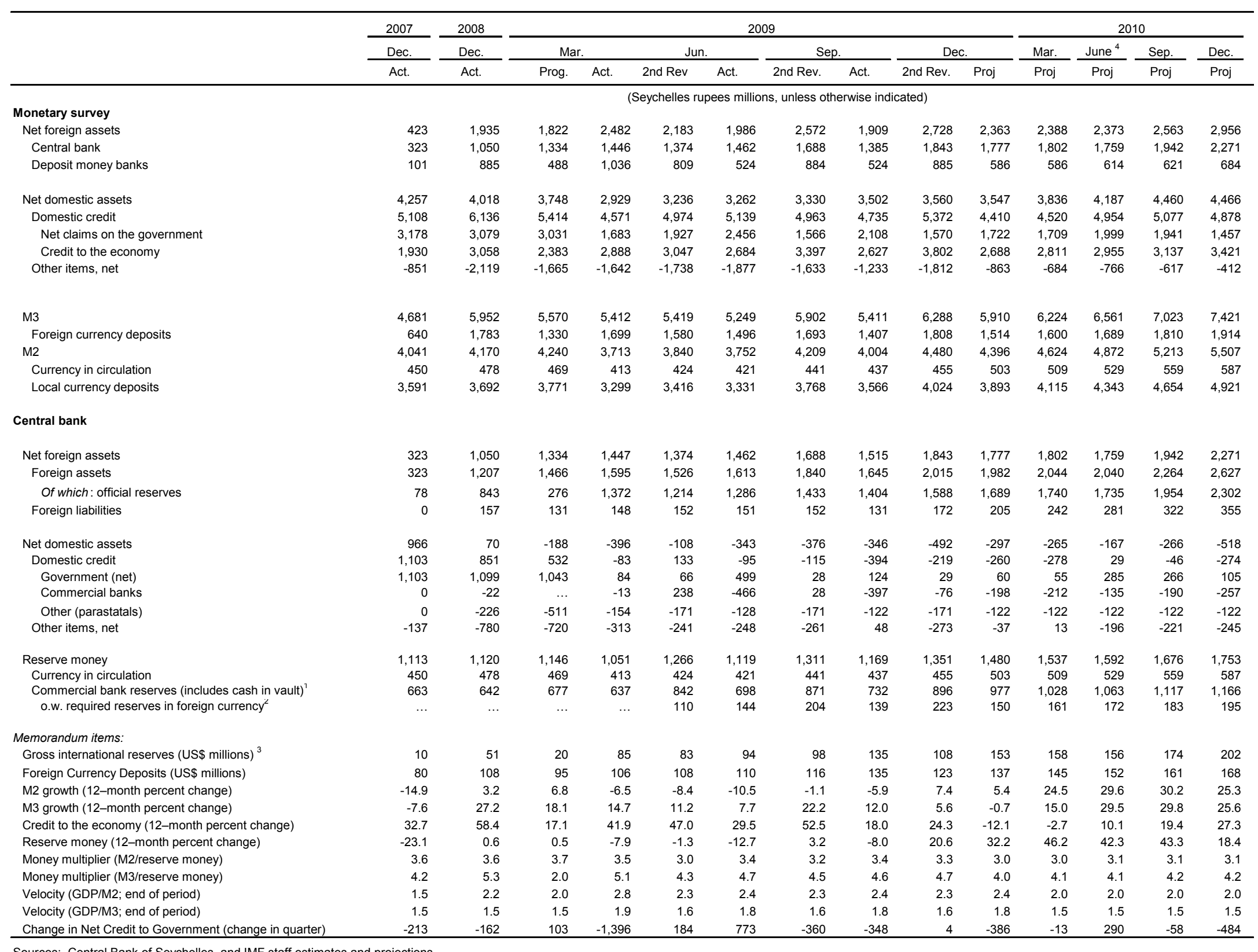

Sources: Central Bank of Seychelles, and IMF staff estimates and projections.

${ }^{1}$ Reserve requirements were lowered from $13 \%$ to $10 \%$ in Q4 2009 .

${ }^{2}$ Starting April 2009, constitution in foreign currency of required reserves on foreign currency deposits were phased in.

${ }^{3}$ Reflects an increase in SDR holdings following SDR allocation of SDR 7.9 million in Q3 2009.

${ }^{4}$ Assumes recapitalization of the CBS by SR 200 million. 
Table 5. Seychelles: Balance of Payments, 2007-14 (Millions of U.S. dollars)

\begin{tabular}{|c|c|c|c|c|c|c|c|c|c|}
\hline & \multirow[t]{2}{*}{2007} & \multirow{2}{*}{$\begin{array}{l}2008 \\
\text { Prel. }\end{array}$} & \multicolumn{2}{|c|}{2009} & \multirow[t]{2}{*}{2010} & \multirow[t]{2}{*}{2011} & \multirow[t]{2}{*}{2012} & \multirow[t]{2}{*}{2013} & 2014 \\
\hline & & & Prog. & Proj. & & & & & \\
\hline $\begin{array}{l}\text { Current account } \\
\text { (Percent of GDP) }\end{array}$ & $\begin{array}{r}-213 \\
-21\end{array}$ & $\begin{array}{r}-412 \\
-45\end{array}$ & $\begin{array}{r}-202 \\
-30\end{array}$ & $\begin{array}{r}-172 \\
-22\end{array}$ & $\begin{array}{r}-321 \\
-33\end{array}$ & $\begin{array}{r}-304 \\
-30\end{array}$ & $\begin{array}{r}-308 \\
-29\end{array}$ & $\begin{array}{r}-286 \\
-26\end{array}$ & $\begin{array}{r}-307 \\
-26\end{array}$ \\
\hline Trade balance & -175 & -379 & -146 & -130 & -263 & -235 & -277 & -237 & -237 \\
\hline Exports of goods & 392 & 502 & 366 & 428 & 540 & 566 & 591 & 616 & 646 \\
\hline Of which: oil re-exports & 190 & 271 & 186 & 174 & 292 & 315 & 336 & 359 & 385 \\
\hline Of which: tuna exports & 184 & 215 & 171 & 198 & 203 & 204 & 206 & 205 & 206 \\
\hline Imports of goods & -734 & $-1,018$ & -608 & -708 & -878 & -909 & -981 & $-1,018$ & $-1,090$ \\
\hline Oil imports & -236 & -391 & -207 & -228 & -333 & -342 & -371 & -401 & -434 \\
\hline FDI-related imports & -153 & -275 & -146 & -153 & -204 & -215 & -244 & -263 & -315 \\
\hline Other & -345 & -352 & -255 & -327 & -341 & -352 & -366 & -354 & -341 \\
\hline Exports of services & 481 & 517 & 372 & 466 & 462 & 486 & 514 & 579 & 657 \\
\hline Of which: tourism earnings & 284 & 270 & 209 & 238 & 250 & 280 & 313 & 355 & 399 \\
\hline Imports of services & -313 & -379 & -276 & -316 & -387 & -377 & -400 & -414 & -449 \\
\hline Income, net & -72 & -103 & -107 & -109 & -106 & -116 & -79 & -95 & -116 \\
\hline Of which: transfers of profits and dividends & -33 & -61 & -64 & -73 & -67 & -82 & -67 & -83 & -103 \\
\hline Of which: interest payments due ${ }^{1}$ & 0 & -33 & -36 & -36 & -32 & -32 & -12 & -14 & -15 \\
\hline Moratorium interest & & 0 & -4 & -5 & -3 & -3 & -3 & -3 & -3 \\
\hline Other interest (scheduled) & & -33 & -31 & -31 & -29 & -29 & -10 & -11 & -12 \\
\hline Current transfers, net & 33 & 70 & 52 & 67 & 48 & 47 & 47 & 46 & 46 \\
\hline General government, net & 10 & 45 & 27 & 35 & 17 & 16 & 16 & 15 & 13 \\
\hline Other sectors, net & 23 & 25 & 25 & 31 & 31 & 31 & 31 & 31 & 32 \\
\hline Capital and financial account & 261 & 119 & 147 & 155 & 291 & 80 & 358 & 400 & 444 \\
\hline Capital account & 8 & 5 & 37 & 53 & 62 & 17 & 17 & 10 & 15 \\
\hline Financial account & 254 & 113 & 110 & 102 & 229 & 63 & 341 & 390 & 429 \\
\hline Direct investment, net & 225 & 350 & 183 & 192 & 245 & 258 & 295 & 318 & 383 \\
\hline Portfolio investment, net & 31 & 1 & 0 & 1 & 1 & 1 & 1 & 5 & 8 \\
\hline Other investment, net & -2 & -238 & -73 & -90 & -17 & -196 & 45 & 67 & 38 \\
\hline Assets & -66 & -3 & -10 & -32 & 16 & 10 & 5 & 5 & 5 \\
\hline Liabilities & 63 & -235 & -63 & -58 & -33 & -206 & 40 & 62 & 33 \\
\hline Government and government-guaranteed & 34 & -105 & -85 & -89 & -55 & -215 & 34 & 34 & 14 \\
\hline Disbursements & 101 & 84 & 29 & 25 & 36 & 36 & 40 & 40 & 20 \\
\hline Project loans & 101 & 84 & 10 & 5 & 16 & 16 & 20 & 20 & 20 \\
\hline Program loans & 0 & 0 & 19 & 20 & 20 & 20 & 20 & 20 & 0 \\
\hline Amortization (Principal payments) ${ }^{1,2}$ & -67 & -189 & -81 & -80 & -52 & -252 & -7 & -6 & -6 \\
\hline Deferred interest ${ }^{1,2}$ & & & 3 & 4 & 1 & 1 & 1 & 0 & 0 \\
\hline Debt forgiveness & & & -37 & -36 & -39 & 0 & 0 & 0 & 0 \\
\hline Parastatals & 42 & 0 & 0 & -2 & -2 & -2 & -2 & -2 & -2 \\
\hline Disbursements & 49 & 0 & 0 & 0 & 0 & 0 & 0 & 0 & 0 \\
\hline Amortization & 0 & 0 & 0 & -2 & -2 & -2 & -2 & -2 & -2 \\
\hline Other & -12 & -130 & 22 & 33 & 24 & 11 & 7 & 30 & 21 \\
\hline of which: SDR Allocation (- = increase) ${ }^{3}$ & & & & -12 & & & & & \\
\hline Net errors and omissions & -8 & 82 & 23 & 40 & 0 & 0 & 0 & 0 & 0 \\
\hline Overall balance & 40 & -212 & -32 & 23 & -30 & -224 & 50 & 114 & 137 \\
\hline Financing & -40 & 212 & -49 & -52 & -39 & -50 & -56 & -61 & -56 \\
\hline Change in net international reserves (increase: - ) & 49 & 38 & -54 & -95 & -39 & -50 & -56 & -61 & -56 \\
\hline Change in gross official reserves (increase: - ) & 49 & 28 & -58 & -102 & -49 & -50 & -50 & -50 & -50 \\
\hline Prospective liabilities to IMF, net & 0 & 10 & 4 & 8 & 10 & 0 & -6 & -11 & -6 \\
\hline Other NFA (increase: -) & 0 & 0 & -10 & -12 & 0 & 0 & 0 & 0 & 0 \\
\hline Exceptional financing & -89 & 174 & 15 & 55 & 0 & 0 & 0 & 0 & 0 \\
\hline Change in arrears (increase: +$)^{4,5}$ & -89 & 174 & -125 & -97 & 0 & 0 & 0 & 0 & 0 \\
\hline Clearance of arrears & $\ldots$ & $\ldots$ & 140 & 152 & 0 & 0 & 0 & 0 & 0 \\
\hline Financing gap & 0 & 0 & 80 & 29 & 69 & 274 & 6 & -54 & -81 \\
\hline Memorandum items: & & & & & & & & & \\
\hline Exports growth, percent & -8 & 28 & -26 & -15 & 26 & 5 & 4 & 4 & 5 \\
\hline Tourism growth, percent & 25 & -5 & -22 & -12 & 5 & 12 & 12 & 13 & 13 \\
\hline Imports growth, percent & 3 & 39 & -40 & -31 & 24 & 4 & 8 & 4 & 7 \\
\hline Exports, percent of GDP & 38 & 54 & 54 & 56 & 46 & 56 & 55 & 55 & 55 \\
\hline Imports, percent of GDP & 72 & 111 & 90 & 92 & 74 & 90 & 92 & 91 & 92 \\
\hline FDI, percent of GDP & 22 & 38 & 27 & 25 & 25 & 26 & 28 & 28 & 32 \\
\hline Gross international reserves (stock, e.o.p.) ${ }^{3}$ & 10 & 51 & 108 & 153 & 202 & 252 & 302 & 352 & 402 \\
\hline Months of prospective imports of goods and services & 0.1 & 0.6 & 1.3 & 1.5 & 1.9 & 2.2 & 2.5 & 2.7 & 3.1 \\
\hline Scheduled public external debt service ${ }^{1,5}$ & 100 & 221 & 114 & 113 & 86 & 286 & 20 & 22 & 23 \\
\hline (Percent of exports of goods and services) & 11 & 22 & 15 & 13 & 8.5 & 27.2 & 1.8 & 1.9 & 1.8 \\
\hline Public external debt ${ }^{1,5,6}$ & 710 & 767 & 733 & 755 & 708 & $\ldots$ & $\ldots$ & $\ldots$ & $\ldots$ \\
\hline (Percent of GDP) & 69 & 83 & 108 & 99 & 72.2 & $\ldots$ & $\ldots$ & $\ldots$ & $\ldots$ \\
\hline Of which: arrears ${ }^{1,4,5}$ & 160 & 322 & 195 & 239 & 231 & $\ldots$ & $\ldots$ & $\ldots$ & $\ldots$ \\
\hline (Percent of GDP) & 16 & 35 & 29 & 31 & 23.5 & $\ldots$ & $\ldots$ & $\ldots$ & $\ldots$ \\
\hline GDP & 1,026 & 921 & 679 & 766 & 981 & 1,008 & 1,069 & 1,118 & 1,185 \\
\hline al Bank of Seychelles; Ministry of Finar & stat & imate & roje & & & & & & \\
\hline $\begin{array}{l}{ }^{1} \text { Projections are based on identified new borrowing and } \\
\text { reduction in the debt stock for Malaysia and South Africa, } \\
\text { restructuring leads to higher rescheduled interest paymer }\end{array}$ & so signe & ed the ac & ement, a & lebt $r$ & casio & $N$ tre & ent ar & $\begin{array}{l}\text { d ider } \\
\text { es. Ar }\end{array}$ & \\
\hline${ }^{2}$ Based on Paris Club agreement, the payments of princ & most int & terest duri & $\lg 2008$ an & ough Ju & $2009 a$ & deferre & & & \\
\hline${ }^{3}$ Seychelles received an SDR allocation of SDR 7.9 milli & SDR 0.4 & million in & August and & tembe & 09 res & ctively. & & & \\
\hline${ }^{4}$ In 2008, includes accelerated promissory notes. & & & & & & & & & \\
\hline
\end{tabular}

CInternational Monetary Fund. Not for Redistribution 
Table 6. Seychelles: Financial Soundness Indicators for the Banking Sector, $2007-2009^{1}$ (Percent, eop)

\begin{tabular}{|c|c|c|c|c|c|c|c|c|c|c|c|}
\hline & \multicolumn{4}{|c|}{2007} & \multicolumn{4}{|c|}{2008} & \multicolumn{3}{|c|}{2009} \\
\hline & Q1 & Q2 & Q3 & Q4 & Q1 & Q2 & Q3 & Q4 & Q1 & Q2 & Q3 \\
\hline \multicolumn{12}{|l|}{ Capital adequacy $^{2}$} \\
\hline Regulatory capital to risk weighted assets & 20.4 & 21.0 & 18.2 & 18.2 & 18.4 & 17.6 & 17.9 & 19.4 & 13.6 & 17.8 & 22.0 \\
\hline Regulatory tier 1 capital to risk weighted assets & 14.8 & 16.1 & 14.8 & 12.9 & 12.9 & 14.0 & 13.4 & 10.4 & 12.9 & 17.1 & 21.3 \\
\hline Capital to assets (net worth) & 6.2 & 6.5 & 6.4 & 6.4 & 6.9 & 6.6 & 7.0 & 8.4 & 9.6 & 8.7 & 9.7 \\
\hline Net tangible capitalization ${ }^{3}$ & 6.2 & 6.6 & 6.5 & 6.5 & 7.0 & 6.6 & 7.1 & 8.4 & 9.6 & 8.7 & 9.8 \\
\hline \multicolumn{12}{|l|}{ Asset quality } \\
\hline Foreign exchange loans to total loans & 24.1 & 25.1 & 28.7 & 30.8 & 32.7 & 32.9 & 31.6 & 50.8 & 50.5 & 43.0 & 41.1 \\
\hline Non-performing loans to gross loans & 3.7 & 2.9 & 2.5 & 2.3 & 2.0 & 2.1 & 2.0 & 2.0 & 2.3 & 4.3 & 4.2 \\
\hline Provision as percentage of non-performing loans & 38.2 & 44.1 & 45.6 & 45.9 & 51.2 & 50.3 & 59.6 & 58.3 & 56.8 & 35.2 & 32.6 \\
\hline \multicolumn{12}{|l|}{ Earnings and profitability } \\
\hline Return on assets (annualised) & 3.9 & 3.4 & 5.0 & 6.6 & 4.3 & 3.4 & 2.8 & 16.1 & 5.3 & 3.0 & -0.2 \\
\hline Return on equity (annualised) & 59.1 & 50.4 & 68.3 & 93.0 & 59.5 & 47.9 & 39.3 & 199.4 & 55.1 & 35.4 & -2.8 \\
\hline Interest margin to gross income & 60.0 & 66.1 & 57.9 & 46.0 & 58.7 & 62.8 & 58.3 & 24.2 & 62.1 & 83.7 & 105.5 \\
\hline Non-interest expense to gross income & 35.5 & 39.7 & 33.3 & 32.3 & 33.2 & 43.5 & 49.4 & 17.8 & 36.4 & 48.7 & 116.5 \\
\hline Net interest margin (annualised) ${ }^{4}$ & 3.6 & 3.7 & 4.3 & 4.5 & 3.8 & 3.8 & 3.1 & 4.8 & 5.1 & 4.8 & 4.2 \\
\hline Net non interest margin (annualized) ${ }^{5}$ & 0.3 & -0.3 & 0.6 & 2.1 & 0.5 & -0.4 & -0.4 & 11.4 & 0.1 & -1.9 & -4.9 \\
\hline Expense to income & 48.5 & 53.5 & 44.8 & 42.2 & 46.6 & 55.9 & 62.5 & 25.5 & 52.3 & 62.2 & 111.2 \\
\hline Interest expense to gross income & 25.2 & 29.5 & 20.8 & 17.2 & 25.0 & 28.0 & 35.0 & 10.2 & 33.2 & 35.8 & 47.1 \\
\hline \multicolumn{12}{|l|}{ Liquidity } \\
\hline Core liquid assets to total assets ${ }^{6}$ & 19.2 & 18.6 & 11.4 & 10.0 & 30.8 & 34.6 & 36.4 & 37.8 & 36.0 & 35.0 & 37.7 \\
\hline Broad liquid assets to total assets ${ }^{7}$ & 54.8 & 55.8 & 44.3 & 39.4 & 59.2 & 60.9 & 60.0 & 55.7 & 50.8 & 53.0 & 57.6 \\
\hline $\begin{array}{l}\text { Liquid assets (broad) to short term liabilities } \\
\text { Liquid assets (broad) to total liabilities }\end{array}$ & $\begin{array}{l}57.3 \\
58.7\end{array}$ & $\begin{array}{l}57.5 \\
60.1\end{array}$ & $\begin{array}{l}46.7 \\
47.8\end{array}$ & $\begin{array}{l}40.8 \\
42.3\end{array}$ & $\begin{array}{l}59.3 \\
63.9\end{array}$ & $\begin{array}{l}61.6 \\
65.5\end{array}$ & $\begin{array}{l}87.1 \\
64.8\end{array}$ & $\begin{array}{l}56.5 \\
61.2\end{array}$ & $\begin{array}{l}53.2 \\
56.2\end{array}$ & $\begin{array}{l}56.2 \\
58.0\end{array}$ & $\begin{array}{l}62.1 \\
63.8\end{array}$ \\
\hline Liquid assets to deposit liabilities & 64.0 & 64.5 & 52.5 & 46.0 & 68.3 & 72.4 & 71.7 & 66.4 & 63.1 & 63.8 & 71.5 \\
\hline \multicolumn{12}{|l|}{ Foreign exchange exposure } \\
\hline Net open foreign exchange position to capital & 2.6 & 14.1 & 28.3 & 28.5 & 24.9 & 31.3 & 21.3 & 58.9 & 44.4 & 32.4 & 20.9 \\
\hline
\end{tabular}

Sourse: Central Bank of Seychelles

${ }^{1}$ Excluding purely offshore banks.

${ }^{2}$ Under new standards of provisioning and capital adequacy.

${ }^{3}$ Defined as: equity capital/(assets-interest in suspense-provisions)

${ }^{4}$ Defined as: (Interest income - interest expense)/average assets

${ }^{5}$ Defined as: (Non interest income - non interest expense)/average assets

${ }^{6}$ Core liquid assets include cash, balances with CBS and deposits with other banks.

${ }^{7}$ Broad liquid assets include core liquid assets plus investments in government securities. 
Table 7. Seychelles: Projected Financing Requirements and Sources, 2008-19 (Millions of U.S. dollars)

\begin{tabular}{|c|c|c|c|c|c|c|c|c|c|}
\hline & 2008 & 2009 & 2010 & 2011 & 2012 & 2013 & 2014 & 2017 & 2019 \\
\hline \multicolumn{10}{|l|}{ External Financing } \\
\hline Gross financing requirements & 443.6 & 487.0 & 448.1 & 629.2 & 393.6 & 371.6 & 385.7 & 346.9 & 368.1 \\
\hline External current account deficit (excl. official transfers) & 456.9 & 201.9 & 337.8 & 319.7 & 324.3 & 300.3 & 320.6 & 299.9 & 309.7 \\
\hline Amortization of public debt (scheduled) ${ }^{1}$ & 188.7 & 85.1 & 60.8 & 259.1 & 13.2 & 10.4 & 8.9 & 7.0 & 8.4 \\
\hline Change in arrears (repayment $=+$ ) & -173.5 & 97.4 & 0.0 & 0.0 & 0.0 & 0.0 & 0.0 & 0.0 & 0.0 \\
\hline Gross reserves accumulation (increase $=+$ ) & -28.5 & 102.4 & 49.2 & 50.0 & 50.0 & 50.0 & 50.0 & 40.0 & 50.0 \\
\hline IMF repurchases and charges & 0.0 & 0.2 & 0.4 & 0.4 & 6.2 & 10.9 & 6.2 & 0.0 & 0.0 \\
\hline Available financing & 443.6 & 458.1 & 379.2 & 311.0 & 342.2 & 383.0 & 421.5 & 374.1 & 360.0 \\
\hline Foreign direct investment & 350.2 & 191.6 & 245.0 & 258.2 & 295.3 & 318.1 & 383.5 & 365.8 & 369.0 \\
\hline Disbursements of program support ${ }^{2}$ & 0.0 & 19.7 & 19.6 & 20.0 & 20.0 & 20.0 & 0.0 & 0.0 & 0.0 \\
\hline Multilateral & 0.0 & 19.7 & 19.6 & 20.0 & 20.0 & 20.0 & 0.0 & 0.0 & 0.0 \\
\hline Bilateral & 0.0 & 0.0 & 0.0 & 0.0 & 0.0 & 0.0 & 0.0 & 0.0 & 0.0 \\
\hline Disbursements of project loans & 84.2 & 5.4 & 15.9 & 15.9 & 20.0 & 20.0 & 20.0 & 20.6 & 20.6 \\
\hline Arrears clearance (inc. arrears forgiveness) & 0.0 & 152.1 & 0.0 & 0.0 & 0.0 & 0.0 & 0.0 & 0.0 & 0.0 \\
\hline IMF & 9.6 & 7.5 & 10.3 & 0.0 & 0.0 & 0.0 & 0.0 & 0.0 & 0.0 \\
\hline Other flows ${ }^{3}$ & -0.4 & 81.8 & 88.5 & 16.8 & 6.9 & 24.8 & 18.0 & -12.3 & -29.6 \\
\hline External flow financing gap & & 28.9 & 68.9 & 318.2 & 51.5 & -11.4 & -35.8 & -27.2 & 8.1 \\
\hline External arrears clearance ${ }^{3}$ & & 152.1 & & & & & & & \\
\hline Total financing need & & 181.0 & 68.9 & 318.2 & 51.5 & -11.4 & -35.8 & -27.2 & 8.1 \\
\hline \multicolumn{10}{|l|}{ Fiscal Financing } \\
\hline \multicolumn{10}{|l|}{ Gross fiscal requirements } \\
\hline Debt service & 258.8 & 154.1 & 110.8 & 313.2 & 45.0 & 40.8 & 39.6 & 42.0 & 44.5 \\
\hline Interest payments due & 70.1 & 76.5 & 56.5 & 54.2 & 31.9 & 30.4 & 30.6 & 35.0 & 37.1 \\
\hline Domestic & 37.4 & 45.3 & 27.4 & 25.2 & 22.7 & 19.4 & 19.0 & 20.7 & 21.7 \\
\hline External & 32.7 & 31.3 & 29.1 & 29.0 & 9.2 & 11.1 & 11.7 & 14.3 & 15.4 \\
\hline External principal repayments due ${ }^{1}$ & 188.7 & 77.6 & 54.2 & 259.1 & 13.2 & 10.4 & 8.9 & 7.0 & 7.4 \\
\hline Source of funds & 258.9 & 75.2 & 61.4 & 79.8 & 47.1 & 34.5 & 96.5 & 92.4 & 88.6 \\
\hline Primary balance & 35.3 & 102.0 & 68.4 & 60.8 & 49.8 & 52.9 & 49.5 & 46.7 & 43.1 \\
\hline Privatization and long-term leases & 23.1 & 14.2 & 22.2 & 22.8 & 21.3 & 15.4 & 15.1 & 0.0 & 0.0 \\
\hline Domestic (net) & -7.3 & -58.7 & -45.9 & -39.8 & -64.0 & -73.8 & 11.8 & 25.1 & 18.8 \\
\hline External disbursements & 84.2 & 25.0 & 35.5 & 35.9 & 40.0 & 40.0 & 20.0 & 20.6 & 20.6 \\
\hline Change in fiscal arrears (net) & 136.4 & -3.2 & -18.8 & 0.0 & 0.0 & 0.0 & 0.0 & 0.0 & 0.0 \\
\hline Other ${ }^{4}$ & -12.7 & -4.1 & 0.0 & 0.0 & 0.0 & 0.0 & 0.0 & 0.0 & 0.0 \\
\hline Fiscal flow financing gap & -0.1 & 78.9 & 49.3 & 233.5 & -2.1 & 6.3 & -56.9 & -50.4 & -44.1 \\
\hline External arrears clearance & & 152.1 & & & & & & & \\
\hline Total financing need & & 231.0 & 49.3 & 233.5 & -2.1 & 6.3 & -56.9 & -50.4 & -44.1 \\
\hline
\end{tabular}

Sources: Central Bank of Seychelles; Ministry of Finance; and IMF staff estimates and projections.

${ }^{1}$ On existing debt and disbursements of new assumed debt, excluding IMF assuming no debt restructuring and no repayment of arrears.

${ }^{2}$ Includes loans and grants.

${ }^{3}$ Includes all other net financial flows and errors and omissions.

${ }^{4}$ Statistical discrepancy. 


\section{Table 8. Seychelles: Fund Disbursements and Timing of Reviews Under the Proposed Three-Year EFF, 2009-12}

\begin{tabular}{|c|c|c|c|c|}
\hline \multirow{2}{*}{$\begin{array}{l}\text { Program } \\
\text { Review }\end{array}$} & \multirow[b]{2}{*}{ Date of availability } & \multirow[b]{2}{*}{ Conditions } & \multicolumn{2}{|c|}{ Amount } \\
\hline & & & (millions of SDR) & (percent of quota) \\
\hline & December 22, 2009 & Board approval of the EFF arrangement. & 0.88 & 10 \\
\hline & March 15, 2010 & $\begin{array}{l}\text { Compliance with end-December } 2009 \\
\text { quantitative performance criteria and } \\
\text { completion of a financing assurances review (if } \\
\text { needed) }\end{array}$ & 2.20 & 25 \\
\hline \multirow[t]{2}{*}{ First } & June 15, 2010 & $\begin{array}{l}\text { Completion of first program review and } \\
\text { financing assurances review (if needed) and } \\
\text { compliance with end-March } 2010 \text { quantitative } \\
\text { performance criteria. }\end{array}$ & 2.20 & 25 \\
\hline & September 15, 2010 & $\begin{array}{l}\text { Compliance with end-June } 2010 \text { quantitative } \\
\text { performance criteria and completion of a } \\
\text { financing assurances review (if needed) }\end{array}$ & 2.20 & 25 \\
\hline \multirow[t]{2}{*}{ Second } & December 15, 2010 & $\begin{array}{l}\text { Completion of second program review and } \\
\text { financing assurances review (if needed) and } \\
\text { compliance with end-September } 2010 \\
\text { quantitative performance criteria. }\end{array}$ & 1.76 & 20 \\
\hline & March 15, 2011 & $\begin{array}{l}\text { Compliance with end-December } 2010 \\
\text { quantitative performance criteria and } \\
\text { completion of a financing assurances review (if } \\
\text { needed). }\end{array}$ & 1.76 & 20 \\
\hline \multirow[t]{2}{*}{ Third } & June 15, 2011 & $\begin{array}{l}\text { Completion of third program review and a } \\
\text { financing assurances review (if needed) and } \\
\text { compliance with end-March } 2011 \text { quantitative } \\
\text { performance criteria) }\end{array}$ & 1.76 & 20 \\
\hline & September 15, 2011 & $\begin{array}{l}\text { Compliance with end-June } 2011 \text { quantitative } \\
\text { performance criteria and completion of a } \\
\text { financing assurances review (if needed) }\end{array}$ & 1.76 & 20 \\
\hline \multirow[t]{2}{*}{ Fourth } & December 15, 2011 & $\begin{array}{l}\text { Completion of fourth program review and a } \\
\text { financing assurances review (if needed) and } \\
\text { compliance with end-September } 2011 \\
\text { quantitative performance criteria) }\end{array}$ & 1.32 & 15 \\
\hline & March 15, 2012 & $\begin{array}{l}\text { Compliance with end-December } 2011 \\
\text { quantitative performance criteria and } \\
\text { completion of a financing assurances review (if } \\
\text { needed) }\end{array}$ & 1.32 & 15 \\
\hline \multirow[t]{2}{*}{ Fifth } & June 15, 2012 & $\begin{array}{l}\text { Completion of fifth program review and a } \\
\text { financing assurances review (if needed) and } \\
\text { compliance with end-March } 2012 \text { quantitative } \\
\text { performance criteria) }\end{array}$ & 1.32 & 15 \\
\hline & September 15, 2012 & $\begin{array}{l}\text { Compliance with end-June } 2012 \text { quantitative } \\
\text { performance criteria and completion of a } \\
\text { financing assurances review (if needed) }\end{array}$ & 1.32 & 15 \\
\hline Total & & & 19.8 & 225 \\
\hline
\end{tabular}


Table 9. Seychelles: Indicators of Fund Credit, 2008-15

(Millions of SDR)

\begin{tabular}{|c|c|c|c|c|c|c|c|c|}
\hline & 2008 & 2009 & 2010 & 2011 & 2012 & 2013 & 2014 & 2015 \\
\hline & \multicolumn{8}{|c|}{ Projections } \\
\hline \multicolumn{9}{|l|}{ Existing Fund credit } \\
\hline Stock $^{1}$ & 6.2 & 8.8 & 8.8 & 8.8 & 5.4 & 1.0 & 0.0 & 0.0 \\
\hline Obligation & 0.0 & 0.0 & 0.1 & 0.1 & 3.5 & 4.5 & 1.0 & 0.0 \\
\hline Disbursements & 6.2 & 2.6 & 0.0 & 0.0 & 0.0 & 0.0 & 0.0 & 0.0 \\
\hline \multicolumn{9}{|l|}{ Proposed SBA/EFF 2} \\
\hline Disbursements & & 3.1 & 8.4 & 6.6 & 4.0 & 0.0 & 0.0 & 0.0 \\
\hline Stock ${ }^{1}$ & & 3.1 & 11.4 & 18.0 & 22.0 & 20.9 & 19.3 & 17.5 \\
\hline Obligations ${ }^{3}$ & & 0.0 & 0.1 & 0.2 & 0.3 & 1.4 & 1.9 & 2.1 \\
\hline Principal (repayments/repurchases) & & 0.0 & 0.0 & 0.0 & 0.0 & 1.1 & 1.6 & 1.8 \\
\hline Charges and interest & & 0.0 & 0.1 & 0.2 & 0.3 & 0.3 & 0.3 & 0.2 \\
\hline Stock of existing and prospective Fund credit 1 & 6.2 & 11.9 & 20.2 & 26.8 & 27.4 & 21.9 & 19.3 & 17.5 \\
\hline In percent of quota & 70.0 & 135.0 & 230.0 & 305.0 & 311.3 & 248.8 & 219.2 & 198.3 \\
\hline In percent of GDP & 1.0 & 2.4 & 3.2 & 4.1 & 4.0 & 3.0 & 2.5 & 2.1 \\
\hline In percent of exports of goods and services & 0.9 & 2.1 & 3.1 & 4.0 & 3.8 & 2.8 & 2.3 & 1.9 \\
\hline In percent of gross reserves & 18.5 & 12.1 & 15.6 & 16.5 & 14.1 & 9.6 & 7.4 & 6.1 \\
\hline \multicolumn{9}{|l|}{ Obligations to the Fund from existing and prospective } \\
\hline Fund arrangements & 0.0 & 0.0 & 0.2 & 0.3 & 3.8 & 5.8 & 2.9 & 2.1 \\
\hline In percent of quota & 0.3 & 0.1 & 2.4 & 3.5 & 42.8 & 66.1 & 32.6 & 23.5 \\
\hline In percent of GDP & 0.0 & 0.0 & 0.0 & 0.0 & 0.5 & 0.8 & 0.4 & 0.3 \\
\hline In percent of exports of goods and services & 0.0 & 0.0 & 0.0 & 0.0 & 0.5 & 0.8 & 0.3 & 0.2 \\
\hline In percent of gross reserves & 0.1 & 0.0 & 0.2 & 0.2 & 1.9 & 2.6 & 1.1 & 0.7 \\
\hline
\end{tabular}

Sources: IMF Finance Department; and IMF staff estimates and projections.

${ }^{1}$ End of period.

${ }^{2}$ Assumes a disbursement under the current SBA arrangement upon completion of the third review and new EFF arrangement of 225 percent of quota (SDR 19.8 million) conditional upon cancellation of the SBA.

${ }^{3}$ Repayment schedule based on repurchase obligations. 
Figure 1. Seychelles: Macroeconomic Developments
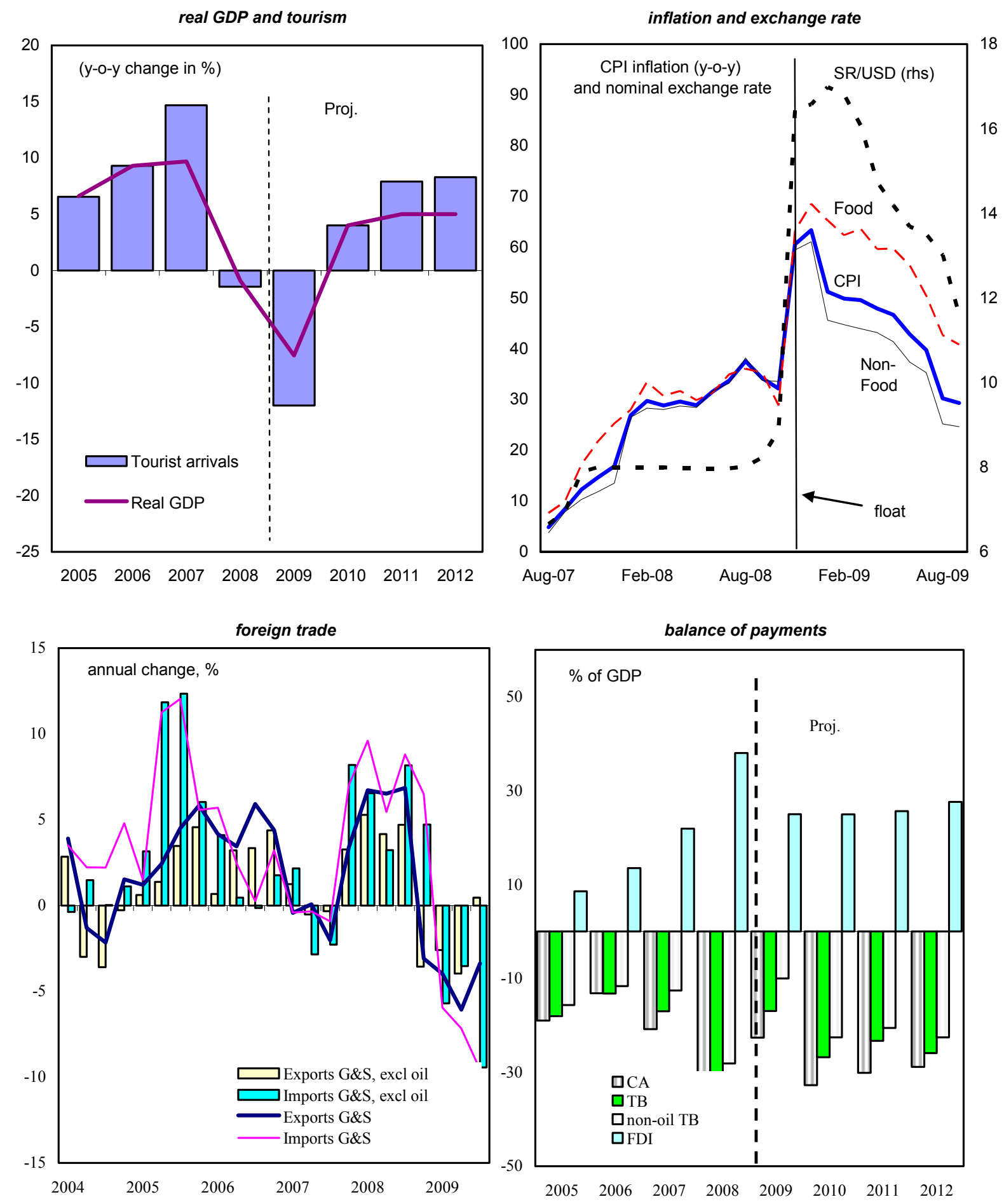

Source: Seychelles authorities; and Fund staff estimates. 
Figure 2. Seychelles: Fiscal Sector
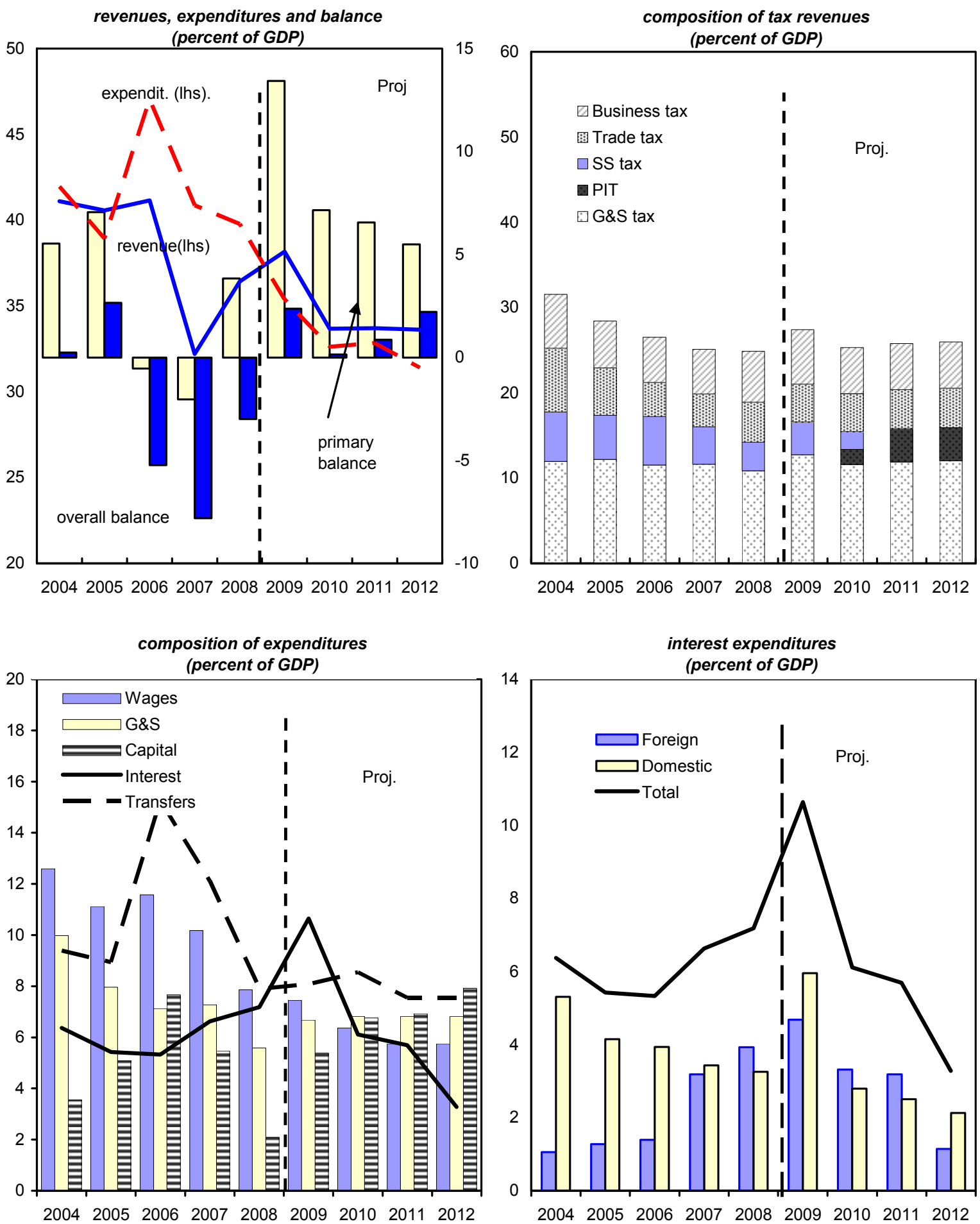

Source: Seychelles authorities; and Fund staff estimates. 
Figure 3. Seychelles: Monetary Sector
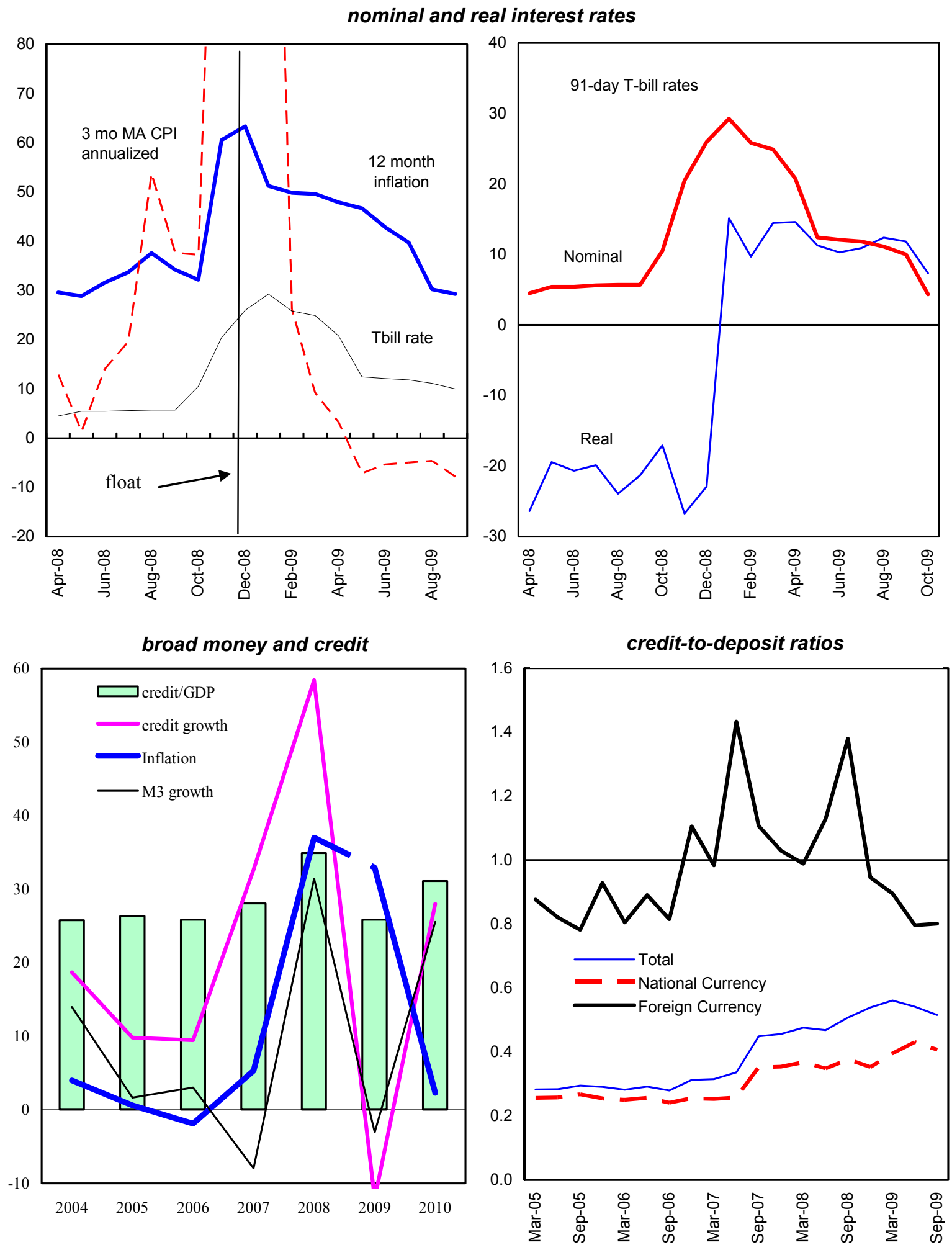

Source: Seychelles authorities; and Fund staff estimates 


\section{APPENDIX I \\ SEYCHELLES: LETTER OF INTENT}

Mr. Dominique Strauss-Kahn

Managing Director

International Monetary Fund

December 3, 2009

Dear Mr. Strauss-Kahn:

1. The attached Memorandum of Economic and Financial Policies for 2009-12 (MEFP) describes Seychelles' performance in the third quarter of 2009, policies for the remainder of 2009, as well as our policies for the 3 year period 2010-12.

2. We request completion of the third review under our Stand-By Arrangement (SBA) and the release of the fourth tranche of SDR 2.2 million (25 percent of quota) there under. All the quantitative performance criteria (PCs) at end-September 2009 were met and all three structural benchmarks at end-September were observed.

3. Following completion of the third review, and associated purchase, we request that the SBA be cancelled and replaced with a 3-year arrangement under the Extended Fund Facility (EFF) in amount of SDR 19.8 million (225 percent of quota) and disbursement of the first tranche of SDR 0.88 million (10 percent of quota). We expect to meet all quantitative PCs and structural benchmarks for end-December 2009.

4. The EFF will support our public debt restructuring process aimed at reestablishing external sustainability; accompany a comprehensive medium-term structural reform strategy aimed at securing macroeconomic stability and raising growth performance; and guide our macroeconomic policies over the next three years.

5. We believe that the economic and financial policies set forth in the MEFP are sufficient to ensure that the objectives of the program will be met. We stand ready to take any further measures that may prove necessary to meet our objectives. We will consult with the Fund on the adoption of these measures, and in advance of revisions to the policies contained in the MEFP, in accordance with the Fund's policies on such consultations. We remain committed to full implementation of the recommendations of the Fund's safeguards assessment of the Central Bank of Seychelles (CBS). 
6. In line with our commitment to transparency, we request that the IMF publish this letter, the MEFP, the technical memorandum of understanding (TMU), and the staff report. We will simultaneously publish these documents in Seychelles.

Sincerely yours,

$$
/ \mathrm{s} /
$$

James Alix Michel

President,

Republic of Seychelles

Attachments: MEFP and TMU 


\section{AtTachment 1}

\section{SEYCHELLES: MeMORANDUM OF ECONOMIC, FINANCIAL AND STRUCTURAL REFORM POLICIES FOR 2009-12}

\section{INTRODUCTION}

1. This document:

a. Reviews macroeconomic and financial performance under our current Stand-By Arrangement (SBA) through end-September 2009.

b. Updates the MEFP of June 16, 2009 for the second SBA program review, detailing our policy commitments through the remainder of 2009 to support completion of the third SBA review.

c. Describes our macroeconomic policies and reform commitments for the three year period 2010-12 under a successor Extended Fund Facility (EFF) arrangement.

\section{Recent Economic Developments ANd Performance Under the Program}

2. The key objectives of our SBA-to stabilize the macroeconomy and initiate the first generation structural reforms-have been achieved. The functioning of our economy has improved significantly following the reforms launched in late 2008. In particular, with the removal of all exchange restrictions has catalyzed substantial improvements in economic efficiency. After a period of turbulence immediately following exchange rate liberalization, the rupee stabilized and has appreciated significantly from its lows early in the year. Inflation has continued at near zero levels since March 2009 and nominal interest rates on short-term T-bills declined to under 5 percent at end-October, consistent with tight fiscal policy and exchange rate trends. The reconstitution of gross international reserves has progressed strongly to US\$136 million (1.3 months of imports) at end-October, in part due to the general SDR allocation (US\$10 million). All quantitative PCs at end-September 2009 were met with margins (Table 1) and we have implemented the measures included in the MEFP for the second SBA review (Table 2).

3. The decline in real GDP growth in 2009 will be less than earlier projected, as the reduction in tourism earnings and related construction activity is attenuating. As a small open economy, Seychelles was hard hit by the global recession and piracy in the Indian Ocean, but there are now early signs of a revival of business activity and employment creation. We now project real GDP to contract by $7 \frac{1}{2}$ percent in 2009 , an improvement on the 10.7 percent contraction projected in June.

4. The government has consistently exceeded its fiscal targets this year with a primary surplus of 13.4 percent of GDP recorded in the first nine months of 2009. Expenditure has been tightly controlled and revenue has held up well despite the difficult 
domestic and external environment. The Public Procurement Act has induced needed discipline in expenditure commitments and the repayment of budget arrears has continued. Social assistance outlays have risen during the year, but have remained well within budget allocations.

5. The Central Bank's (CBS) reserve money targeting framework is achieving its objectives and the CBS gained additional flexibility with the introduction of new indirect policy instruments. In addition to weekly deposit auctions and a standing credit facility, the CBS's portfolio of treasury bills have been actively used in open market operations. In line with best international practices, repo and reverse repo transactions are gradually becoming the main policy tools for liquidity management. The rapid disinflation in 2009 has provided room for a measured easing of monetary policy. Reserve requirements have been reduced from 13 percent of deposits to 10 percent as of October 1, 2009 and the local asset ratio abolished. While benchmark interest rates on government securities fell sharply from the peak of 30 percent in January to 5 percent in October, credit growth has been slow to recover due to the contraction in the real economy.

6. The floating exchange regime has contributed to the rapid stabilization of the economy and has eased the burden on monetary policy. Following a period of overshooting after the float, the rupee has steadily appreciated as banks have unwound large open foreign exchange positions and real interest rates remained strongly positive through early October. The CBS's partly sterilized interventions have been limited to smoothing excessive market volatility and ensuring the sound functioning of the market. The nominal exchange rate appreciated sharply in early October, in part due to continued buildup of the government's cash balances through T-bill sales, which proved unnecessary. In response, treasury cash surpluses were sharply reduced in early October and the foreign exchange market has since stabilized.

\section{Our structural reform agenda is gaining momentum, with all structural} benchmarks implemented as planned (Table 3). A new Public Enterprise Monitoring and Control Act was approved by the National Assembly in September, legislating financial reporting obligations and corporate governance guidelines for all public entities. The introduction of a treasury single account has produced major improvements in cash and expenditure management. External financial audits of the seven largest public enterprises have been completed and management audits of major public entities have also progressed, catalyzing major changes in business practices in some key public enterprises (notably, the utilities company (PUC), the public transport company (SPTC) and Air Seychelles). New Central Bank and Financial Institutions Acts have been put in place, strengthening the framework for monetary policy and financial sector regulation and development.

8. The CBS has made considerable progress implementing the recommendations of the 2008 Safeguards assessment report. During 2009 the CBS Board approved a policy that will ensure adequate external audit quality in future years. It also has adopted an internal audit charter. The internal control framework was strengthened by: (i) the reorganization of 
the Banking Services Division to allow for the segregation of the accounting and foreign reserves management functions and reporting; (ii) the establishment of an Investment Committee and adoption of an asset-liability management framework for strategic allocation of reserves; and (iii) the appointment of an Operational Risk Committee to oversee the development and maintenance of the CBS' operational risk management framework. The CBS is in the final stages of adopting IFRS-based financial reporting for the 2009 accounts.

9. The CBS strengthened the capital requirement for banks and adopted a system of prompt-corrective action. The system is driven by the CAMELS bank rating system and includes triggers for strong and aggressive action for banks that fail to meet the required capital levels. The CBS' Financial Services Supervision Division was reorganized with separate onsite, offsite, policy and foreign exchange sections.

10. We have made good progress in normalizing relations with external creditors and restructuring our unsustainable external debt. We successfully negotiated a debt restructuring with the Paris Club in April 2009, signed a bilateral rescheduling agreement with Malaysia on comparable terms and have also made good progress with private creditors. Following active discussions with all creditor groups, we are close to launching a debt exchange offer for Eurobond and amortizing note holders on terms compatible with Seychelles' limited payments capacity, and comparable to those agreed with Paris Club creditors. The offer will be supported by a US\$10 million policy-based Guarantee Operation from the African Development Bank, reducing the coupon on the exchange bond and providing additional comfort to creditors. We expect the debt exchange with commercial bondholders to be completed by mid-January 2010. Discussions with commercial banks have proceeded in parallel and restructuring terms agreed on a few loans. Some banks may participate in the exchange bond offer.

\section{Policies FOR THE REMAINDER OF 2009}

\section{We remain committed to implementing the policies and meeting the structural} and quantitative targets for end-December 2009 described in our previous MEFP. Our forecasts are for inflation to remain in the low single digits, allowing further measured easing of monetary policy. The external current account deficit is expected to narrow, with exports of tourism services expected to increase as the global economy recovers and additional hotel accommodation comes on line. Gross reserves are expected to rise further to US\$153 million by end-2009 (1.4 months of imports).

\section{We will meet the $\mathbf{2 0 0 9}$ program primary surplus target, adjusted for foreign} financing of 13.4 percent of GDP. The government's over performance in the first 3 quarters has provided the fiscal space for a supplementary appropriation of about 2 percent of GDP to meet emergency expenditure needs, the most notable components of which are emergency financing for Air Seychelles ( 0.3 percent); departure payments for workers whose jobs are being outsourced in 2010 (0.3 percent); enhanced cofinancing of private sector tourism marketing ( 0.2 percent); the rehabilitation of PUC ( 0.4 percent); a targeted voluntary 
departure scheme (0.1 percent); and strengthening the capacity of the Seychelles Revenue Commission (SRC) to deliver tax reform (0.1 percent). Our overall fiscal performance, coupled with falling interest rates and increased external financial assistance, will allow us to reduce domestic debt by $5 \frac{1}{2}$ percent of GDP in 2009 . We are continuing to strengthen our treasury management capacity in close collaboration with the CBS.

13. The CBS will closely monitor the impact of recent monetary easing. Inflation has remained near zero for some time and the exchange rate appreciation of the past few months is still working its way through domestic prices. While the reserve money target for December has been raised moderately, the CBS stands ready to tighten monetary policy at the first signs of a pick up in inflation.

14. Our structural reform efforts are progressing apace and we expect to meet the structural benchmarks set for end-December 2009 under our SBA. Business tax reforms are being finalized for introduction from January 1, 2010 and we are working towards preparation of more comprehensive budget documentation for 2010. Further expenditure reforms and rationalization, bolstered oversight and monitoring of the public entities and enterprises, and strengthened financial sector supervision are being implemented. We have prepared a medium-term debt strategy and annual borrowing plan to accompany the 2010 Budget.

\section{Medium-Term Macroeconomic ANd Structural Reform Policies}

\section{The major objectives of our EFF-supported program will be to:}

a. preserve macroeconomic stability;

b. achieve external sustainability; and

c. improve economic efficiency and durably raise growth through the implementation of a second generation of structural reforms.

16. Our ambitious structural reform agenda aims to transform the economy by institutionalizing higher governance standards, rationalizing the public sector and improving the business environment. We will continue to remove distortions, modernize economic laws and regulations, and reduce and refocus the role of the state in the economy from operator to facilitator and regulator. We will pursue a significant reinforcement of monitoring and control over parastatal bodies to address major financial risks to the stability of public finances and choke-points to growth. Some of the key reforms-public enterprise restructuring, tax reform, strengthened public financial management — were initiated under our current SBA.

\section{This reform strategy serves as a coordinating function for donors and} stakeholders. Our reform program is supported by contributions from several multilateral (AfDB, IBRD, EU, UNDP) and other development partners through development policy 
loans, public expenditure reviews, related programs and technical assistance. Most notably, we are working with the World Bank to elaborate a strategy for improving the business climate and modernizing the labor law.

18. The EFF provides a macroeconomic framework to guide fiscal and financial policies over the next three years. After the shocks of 2008-09, we expect the economy to start a sustained recovery from 2010 . There are positive signs that tourism and related services - the main drivers of growth - commenced a gradual recovery in the second half of 2009, and FDI is expected to rebound in 2010 with several large projects in the pipeline. The investment would be supported by a successful public external debt restructuring in late2009/early-2010 that would reduce country credit risk. Real GDP growth is projected to recover to 4 percent in 2010 and to 5 percent in 2011-12, while inflation is expected to remain in the low single digits. The external current account deficit is expected to widen again in 2010, due to higher growth and FDI, but narrow over the medium term as tourism earnings recover. Foreign exchange reserves are projected to rise gradually to about $2 \frac{1}{2}$ months of import coverage by end-2012.

\section{A. Fiscal Policy}

19. The overarching goals of fiscal policy are to put public finances on a sustainable path, while creating the fiscal space to raise public investment and support our targeted social safety net. Primary fiscal surpluses will be targeted at 7 percent of GDP in 2010, declining to about $4 \frac{1}{2}$ percent by 2012 , levels that are consistent with achieving our mediumterm sustainability objective, assuming full external debt restructuring. This profile will allow us to reduce domestic public debt significantly, releasing resources to support private sector development.

\section{0 budget}

20. Following the sharp tightening in 2009, an easing of the fiscal stance to a 7 percent primary surplus is targeted for $\mathbf{2 0 1 0}$. We will implement the first phase of the medium-term tax reform with the 2010 Budget. The tax reforms are broadly revenue neutral in 2009 but overall revenue will be lower on account of significant one-off revenues and the exchange rate appreciation effect totaling almost 4 percent of GDP. We will also continue to strengthen the revenue commission and its tax administration functions to improve governance, efficiency, and effectiveness. A conservative revenue forecast is assumed in the program as there are uncertainties regarding the underlying tax base, the response to the tax reform, and the large nominal appreciation of the rupee in 2009, which are expected to result in realized revaluation losses primarily for the financial sector. Should overall revenue exceed projections in 2010, we intend to save a portion and use the rest for additional urgent infrastructure spending; the appropriate savings/spending balance would be determined in consultation with IMF staff at the time of the first program review under the EFF, determined in the context of the macroeconomic situation then prevailing. 


\section{On the expenditure side, rationalizing the size of the public sector and} reinforcing control over public finances will create the space for higher capital investment. Building on our tight control of government expenditures in 2009, recurrent expenditures will be reduced further as a percentage of GDP in the 2010 Budget. This will allow us to increase capital expenditure to 7.7 percent of GDP in 2010 (including the spending contingency), directed primarily at urgent rehabilitation of the electricity and water infrastructure that was curtailed in the past by foreign exchange restrictions and, if left unaddressed, would threaten our projected growth recovery. The budget will also include an expenditure contingency of about 1 percent of GDP, which has been earmarked for investment projects that will proceed as long as the primary fiscal position remains on track.

\section{Tax reform}

\section{A fundamental medium-term reform of the tax system will be launched with} the 2010 Budget. Our objective is to have a simple, fair, and equitable system, which will promote growth, improve self-compliance, and level the playing field for investors. We will launch a communication campaign to explain tax system changes and consult extensively with stakeholders before design features are settled. The reform is aimed to be broadly revenue neutral over the medium term, with a phased reduction in business tax rates compensated by a broadened direct and indirect tax base, including through the elimination of exemptions. The reform has three pillars:

- $\quad$ Broadening the business tax base by removing exemptions and sectoral preferences (tourism and fishing), eliminating thresholds for corporations and reducing the threshold for noncorporate entities, addressing accelerated capital depreciation, introducing a thin capitalization norms. Business tax rates will be lowered in steps from 2010 through to 2012 to promote competitiveness and achieve harmonization across sectors. The revised Business Tax Act will be introduced with the 2010 Budget and will come into effect on January 1, 2010.

- A withholding-based personal income tax (PIT) on domestic sourced revenue for nationals and resident expatriates will also be introduced with the 2010 Budget, replacing the social security contribution from July 1, 2010. This will broaden the income tax base from withholding on wages to include domestic-sourced income, dividends, and some fringe benefits, not captured under the Business Tax Act. The new PIT will also include a rebate for low income earners.

- Introduction of a single rate VAT from January 1, 2012, replacing the current multiple rate GST, broadening the indirect tax base and improving both the efficiency of the indirect tax system and external competitiveness. Important steps in this direction will be taken in the 2010 Budget, including expanding the GST base to a broader range of services and the reduction of cascading effects through the levying of GST once-on either imports or sales. 
We aim to have a level playing field on taxation in place by January 2012. The granting of new tax incentives under the Tourism Incentive Act, Agriculture and Fisheries Incentive Act, and the Investment Promotion Act will be strictly limited and any new incentives, or extensions to existing ones, granted after October 1, 2009 will lapse on December 31, 2011. In addition, we are in discussions with established operators under these incentive regimes with a view to bringing them into the new tax regime as soon as possible. It is our aim to phase out these concessions by January 2012, concurrent with the introduction of the VAT.

\section{We are also modernizing and reinforcing revenue administration in line with recommendations by the IMF's Fiscal Affairs Department.}

\begin{tabular}{|c|c|}
\hline Action & Timing \\
\hline Revenue Administration-General & \\
\hline $\begin{array}{l}\text { SRC Governance Reform: } \\
\text { - } \quad \text { Implement an integrated function based structure for domestic tax } \\
\text { operations, with an audit group that is specifically focused on large } \\
\text { taxpayers. } \\
\text { - } \quad \text { Create a dedicated project reform office. } \\
\text { - } \quad \text { Establish corporate-level staff affairs and internal audit units. }\end{array}$ & December 2009 \\
\hline - Introduce new Revenue Administration and Business Number Acts. & January 2010 \\
\hline Customs Administration & \\
\hline - Develop a customs reform strategy and implementation plan. & March 2010 \\
\hline - Migrate to ASYCUDA World. & $2^{\text {nd }}$ half of 2010 \\
\hline $\begin{array}{l}\text { - Create two new headquarters-type units in the customs division for program } \\
\text { development/planning and enforcement/investigations. }\end{array}$ & March 2010 \\
\hline - Introduce new Customs Management Act, including HS codes. & June 2010 \\
\hline - Develop trade administration expertise and develop a WCO strategy. & 2010 \\
\hline
\end{tabular}




\section{Tax policy}

\begin{tabular}{|c|c|}
\hline Action & Timing \\
\hline \multicolumn{2}{|l|}{ Business Tax } \\
\hline - $\quad$ Introduce new Business Tax Act & January 2010 \\
\hline - $\quad$ Eliminate retail shops from the International Trade Zone (ITZ) & July 2010 \\
\hline \multicolumn{2}{|l|}{ Personal Income Tax (Pay as you go withholding) } \\
\hline - $\quad$ Introduce new PIT legislation to replace Social Security contribution. & July 2010 \\
\hline \multicolumn{2}{|l|}{ Indirect taxation } \\
\hline $\begin{array}{l}\text { - Extend full GST to all consultancy and corporate service provider service } \\
\text { fees. }\end{array}$ & January 2010 \\
\hline - $\quad$ Reinstitute phased rise in GST on tourism to $15 \%$ with initial rise to $12 \%$ & November 2010 \\
\hline $\begin{array}{l}\text { - } \quad \text { Reduce cascading by eliminating GST on imports for all business that are } \\
\text { liable for GST on their sales }\end{array}$ & January 2010 \\
\hline - $\quad$ Eliminate the retail mark-up to calculate the GST and raise rates to $15 \%$ & January 2010 \\
\hline \multicolumn{2}{|l|}{ Trade Taxes and Excises } \\
\hline $\begin{array}{l}\text { - Launch an excise regime to identically tax imported and domestically } \\
\text { produced excisable commodities. }\end{array}$ & July 2010 \\
\hline $\begin{array}{l}\text { - Partial conversion of trade taxes on petroleum, cigarettes, and alcohol to } \\
\text { excise duties consistent with commitments under the COMESA. }\end{array}$ & January 2010 \\
\hline - $\quad$ Extend partial excises on petroleum to PUC and on domestic aviation fuel & January 2010 \\
\hline - $\quad$ Reduce by one-half rebates on fuel, except for registered artisanal fishermen. & January 2010 \\
\hline
\end{tabular}

\section{Public financial management and expenditure reform}

24. We will continue to strengthen the public financial management framework. Modernizing and strengthening the treasury function will be important to consolidate and optimize the use of government cash balances and improve oversight by the Ministry of Finance of spending units. The introduction of a Treasury Single Account represents a major step forward in our capacity to both monitor and control public expenditure and manage the government's cash balances, with related benefits to the central bank's liquidity management operations. This measure has also revealed the need for a new chart of accounts, which we are aiming to introduce with the 2011 Budget.

\section{We will implement expenditure rationalization measures recommended in the} World Bank's Public Expenditure Review (PER), starting in 2010. In 2009 government employment was reduced by some 2,500 employees (about 15 per cent of the public sector workforce) through a voluntary departure and early retirement scheme. We are now in the process of articulating strategic plans for each government sector, initially focusing on health and education, which will inform further efforts to improve the efficiency of government 
service provision. Based on the findings of a high-level government task force, the functions of the civil service are being progressively streamlined with a view to eliminating inefficiencies and duplication, overstaffing in ministries and public entities, and outsourcing ancillary services to the private sector. Initial measures in this regard are the outsourcing of 650 noncore support staff, the removal of vacant posts from the government payroll and a targeted voluntary departure scheme for the security forces. Public sector wage policy is also being reformed through the introduction of a new wage grid that will consolidate allowances, establish a clearer link between remuneration and performance, realigning incentives, while containing growth in the overall public sector wage bill.

\section{Our social safety net will continue to strike a balance between targeted income} support and retaining incentives to work. Following the elimination of universal subsidies in 2008, the government introduced a means-tested social safety net to ensure that lowincome households are able to meet a basic standard of living. Reflecting the new targeted approach to social assistance, we are preparing a merger of Social Security Fund administration into the Social Welfare Agency by July 1, 2010 with the view to securing administrative efficiencies and savings. We are also working, with World Bank assistance, to fine-tune the targeting of our social safety net and to establish linkages to the minimum wage so as to preserve the incentive to work. We are also expanding training programs for the unemployed and those exiting the public service through the voluntary departure scheme to ensure that any period of unemployment is transitory.

\section{We will also improve transparency and strengthen institutional capacity in the} area of fishing licenses, recognizing the importance of this sector to our economy. In the interests of transparency and public accountability, we will start to disclose the details of all fishing license agreements in 2010. Commensurately, we will work with the World Bank to build an economic and financial model to support more informed fishing licensing and natural resource management policies.

\begin{tabular}{|c|c|}
\hline Action & Timing \\
\hline - Introduce a new public sector wage grid. & January 2010 \\
\hline - Fine-tune targeting of social safety net with World Bank assistance. & 2010 \\
\hline $\begin{array}{l}\text { - Complete the merger of the administrations of Social Security Fund into the } \\
\text { Social Welfare Agency. }\end{array}$ & July 2010 \\
\hline $\begin{array}{l}\text { - Systematic disclosure of the fishing license fees, details of all fishing } \\
\text { agreements and payments, industry related agreements signed by government } \\
\text { and fish catch statistics. }\end{array}$ & March 2010 \\
\hline $\begin{array}{l}\text { - Develop an economic and financial model, with World Bank assistance, of the } \\
\text { fisheries to support more informed licensing and natural resource management } \\
\text { policies }\end{array}$ & 2010 \\
\hline $\begin{array}{l}\text { - Reform the Pension Fund funding formula with the assistance of the World } \\
\text { Bank to link with wage levels and provide adequate and sustainable funding. }\end{array}$ & 2010 \\
\hline
\end{tabular}




\section{Budget preparation and presentation}

\section{Our fiscal policy objectives will be supported by strengthened budget processes} and improved budget documentation, as recommended by the IMF's Fiscal Affairs Department. Starting with the 2011 Budget, we will move to a medium-term budgeting framework, presenting the government's fiscal and economic projections over a three-year period. The 2011 Budget process will commence in March 2010 with Cabinet's discussion of a budget strategy document, setting the fiscal context and establishing the government's priorities for the budget. Ministries and other budget dependent entities will then submit their recurrent and capital expenditure proposals to the Ministry of Finance using a standard template that will require detailed costings to be produced over the three-year budget estimates period. The Ministry of Finance's capacity to scrutinize these expenditure proposals will also be enhanced through increased resourcing, the introduction of formal expenditure review procedures and detailed reconciliation of budget estimates with quarterly and year-end outcomes. The recently created financial analysis unit at the SRC will be

\begin{tabular}{|c|c|}
\hline \multirow{2}{*}{\begin{tabular}{|l|} 
Action \\
Budget \\
\end{tabular}} & Timing \\
\hline & \\
\hline $\begin{array}{l}\text { Extend budget coverage for } 2010 \text { to full presentation of revenues, } \\
\text { expenditure and financing, and include discussion of macroeconomic } \\
\text { assumptions and objectives, fiscal targets and objectives, and an annual } \\
\text { borrowing plan, supported by a medium-term debt strategy. }\end{array}$ & December 2009 \\
\hline $\begin{array}{l}\text { - Include a full break down of the revenue and expenditures of budget } \\
\text { dependent agencies as an annex to the } 2010 \text { Budget tables }\end{array}$ & December 2009 \\
\hline $\begin{array}{l}\text { - Determine budget treatment of all budget-dependent agencies for } 2011 \\
\text { budget }\end{array}$ & June 2010 \\
\hline $\begin{array}{l}\text { - Build capacity in the Ministry of Finance to analyse expenditure } \\
\text { proposals }\end{array}$ & $2010-11$ \\
\hline - $\quad$ Transfer financial analysis unit from SRC to Ministry of Finance & June 2010 \\
\hline - $\quad$ Cabinet approval of 2011 Budget strategy. & June 2010 \\
\hline $\begin{array}{l}\text { - The Ministry of Finance to circulate a standard template requiring all } \\
\text { ministries and budget dependent bodies to submit an analysis of mid- } \\
\text { year expenditure outcomes relative to budget and updated forecasts. }\end{array}$ & July 2010 \\
\hline $\begin{array}{l}\text { - Introduce budget submission protocols and procedures for spending } \\
\text { ministries and budget dependent agencies for the } 2011 \text { budget }\end{array}$ & July 2010 \\
\hline $\begin{array}{l}\text { - Produce a consolidated fiscal table for general government, incorporating } \\
\text { all budget dependent agencies. }\end{array}$ & June 2010 \\
\hline - Adopt a new chart of accounts for the 2011 budget & November 2010 \\
\hline - $\quad$ Revise the Financial Instructions and Accounting Manual & March 2011 \\
\hline - Introduce a new Public Finance Law & September 2011 \\
\hline
\end{tabular}


transferred to the Ministry of Finance in 2010 and we will work to increase its capacity.

29. We are also working towards a more complete presentation of the budget for 2010. We will publish a full breakdown of the revenue and expenditures of budget dependent agencies as an annex to the 2010 Budget tables, increasing the transparency of the government's financial commitments. We will determine the budget treatment of all other budget-dependent agencies - moving from reduced form to full presentation in the fiscal tables where applicable - for the 2010 Mid-Year Budget Review. We will then prepare consolidated general government accounts for the 2011 Budget.

\section{B. Public Enterprise Reform}

30. The government has adopted a public enterprise reform strategy aimed at making the sector more efficient, transparent and accountable. It includes a detailed work plan for the new Public Enterprise Monitoring Division (PEMD) directed at reinforcing the monitoring and control of public enterprises, strengthening governance, reducing dependence on public funding and minimizing financial risks to the state. The legal basis for this work was established with the recent approval of the Public Enterprise Monitoring and Control Act (the Act) by the National Assembly, mandating strengthened reporting arrangements for public enterprises and setting uniform governance rules (e.g. appointment of the Chief Executive Officer, establishment of the Board of Directors and the role of supervisory Ministries). The Act also requires each public enterprise to prepare a statement of corporate intent to ensure that the objectives of Management, the Board of Directors and the government as shareholder are in full alignment. Performance targets will also be established, where possible, against which the public enterprise will be assessed.

\section{We are also taking steps towards further rationalization of public sector}

ownership. The government has privatized a number of state-owned enterprises over the last two years, but there is scope to go further. The Public Enterprise Monitoring Division is finalizing strategic assessments for each public entity-drawing on recently concluded external financial audits and management audits - evaluating the case for public ownership and making recommendations to the government. We have also tendered for expert services to conduct an efficiency review and strategic assessment of the options for the future of the national airline and its ancillary services.

\section{The government, together with the CBS, is developing an exit and consolidation} strategy for publicly-owned financial institutions. Privatization of Seychelles Savings Bank and Nouvobanq would bring new technology, strengthen management and fresh capital to our banking system, stimulate competition and enhance the quality of bank services. The process will commence in December 2009 with the contracting of specialist privatization expertise through an open international tender with the objective of bringing both institutions to the market before end-September 2010, market conditions permitting. We have sought the assistance of the FIRST initiative to assess the government's strategic options with regard to three nondeposit taking institutions - the Development Bank of Seychelles, Housing Finance 
Company, and the Concessional Credit Agency with a view to their consolidation and/or privatization. The CBS and PEMD have already started to lay the groundwork for this assessment.

\begin{tabular}{|c|c|}
\hline Action & Timing \\
\hline $\begin{array}{l}\text { - Cabinet approval of a public enterprise reform strategy and work } \\
\text { plan for the PEMD }\end{array}$ & December 2009 \\
\hline - $\quad$ Begin establishing performance benchmarks for public enterprises & December 2009 \\
\hline $\begin{array}{l}\text { Engage IFC or launch a tender for an advisor for the privatization of } \\
\text { Nouvobanq and Seychelles Savings Bank }\end{array}$ & December 2009 \\
\hline $\begin{array}{l}\text { - Prepare a strategic assessment and reform action plan for each major } \\
\text { public enterprise. }\end{array}$ & $2010-11$ \\
\hline $\begin{array}{l}\text { Elaborate strategy to consolidate and/or privatize the Development } \\
\text { Bank of Seychelles, Housing Finance Company, and the } \\
\text { Concessional Credit Agency. }\end{array}$ & 2010 \\
\hline - $\quad$ Air Seychelles: & \\
\hline - Implement management audit action plan & November 2009 \\
\hline $\begin{array}{l}\text { - Launch tender for strategic options assessment and efficiency } \\
\text { review }\end{array}$ & November 2009 \\
\hline $\begin{array}{l}\text { - Adopt an action plan to implement the recommendations of the } \\
\text { strategic assessment and efficiency review }\end{array}$ & June 2010 \\
\hline $\begin{array}{l}\text { - Complete management audits of additional public entities: } \\
\text { - Seychelles Tourism Board (STB) } \\
\text { - Seychelles Fishing Authority (SFA) } \\
\text { - National Arts Council (NAC) } \\
\text { - Seychelles Trading Company (STC) } \\
\text { - Seychelles Civil Aviation Authority (SCAA) } \\
\text { - Seychelles International Business Authority (SIBA) } \\
\text { - Nouvobanq } \\
\text { - Seychelles Savings Bank (SSB) } \\
\text { - Development Bank of Seychelles (DBS) }\end{array}$ & 2010 \\
\hline - $\quad$ Formalize all government loans to public entities & January 2010 \\
\hline
\end{tabular}

\section{Public Debt Restructuring and Management Strategy}

33. We are committed to continuing our good faith efforts to achieve a comprehensive public external debt restructuring, consistent with our medium-term payments capacity and the Fund's lending into arrears policy. We will ensure 
information transparency, inter-creditor equity, and dialogue with all creditor groups. As was recognized by Paris Club creditors, immediate and sizable cash flow relief in the short- to medium-term, and a sustained reduction of our debt service burden are necessary. This needs to be supplemented by significant balance of payments support from our international partners.

34. The principal objective of our medium-term debt management strategy is to place public debt on a sustainable path. The strategy approved by Cabinet in November is a commitment to achieving this through the pursuit of prudent fiscal and monetary policies and securing a restructuring of public external debt that is consistent with Seychelles' limited payments capacity. We will also ensure that the government's financing needs and payment obligations are met on a timely basis, at the lowest possible cost consistent with a prudent degree of risk. We will update our debt management strategy annually, or following major events that impact public debt, notably after the debt restructuring is completed. We will also implement the annual borrowing plan for 2010 as approved with the 2010 Budget.

\section{An updated debt sustainability analysis (DSA) produced by IMF staff indicates} that our medium-term fiscal targets and the securing of public external debt restructuring will place our external debt position on the path to sustainability. However, bound tests show that Seychelles will remain highly vulnerable to shocks and demonstrate the importance of adhering to the fiscal path defined by our macroeconomic framework.

\section{Foreign Trade Regime}

36. We intend to implement the COMESA common external tariff within the 5 year period from our admission this year (2013), as per our market access offer made under the interim EPA signed in August 2009. We have also reactivated our application to join the WTO and are actively pursuing discussions to that end.

\section{E. Monetary, Exchange Rate, and Financial Sector Policies}

37. Price stability will remain the primary objective of monetary policy. Our success in containing inflation over the past year and significant appreciation of the rupee provided scope to loosen monetary policy in the third quarter of 2009 and raise the reserve money target for December 2009. We are monitoring credit and price developments closely and stand ready to tighten monetary policy should inflationary pressures re-emerge. In line with our policy to abolish all direct monetary instruments, we will continue to gradually reduce the reserve requirement as conditions permit.

38. Monetary policy will continue to be based on reserve money targeting through the use of indirect instruments. The CBS has recently added repurchase agreements (repos) and credit auction arrangements (CAA) to its range of monetary instruments. Beginning in 2010, we will introduce a Standing Deposit Facility (SDF) to complement the current 
overnight credit facility. Once this is in place, the CBS will use repos to guide short-term interest rates. In addition, foreign exchange swaps and forwards will also be put in place to diversify and further deepen the foreign exchange market.

39. Development of money markets is an important priority. 91-day T-bills have been established as the benchmark reference rate for the pricing of securities. However, there is no secondary market and the primary market remains shallow. Direct nonbank participation in Treasury bill auctions will continue until the infrastructure for a properly functioning secondary market is in place. Once this is achieved, we will introduce a primary dealer system whereby participation in Treasury bill auctions will be restricted to banks, who will bid on their own behalf and on behalf of their customers.

40. Coordination between monetary and fiscal policy will remain key to an efficient liquidity management framework. To this end, we will continue to make extensive use of the recently established liquidity forecasting and reserve money programming framework. The CBS and Ministry of Finance will work closely on the T-bill issuance profile-and subsequent communication to market participants - to promote the smooth functioning of the market. The CBS will also formalize the arrangements for its use of government securities as a monetary policy instrument through a memorandum of understanding with the Ministry of Finance.

\section{We remain committed to a floating exchange rate regime, which has served} Seychelles well during the initial phase of our economic reforms. The CBS will only intervene in the foreign exchange market to smooth out excessive volatility and ensure orderly market conditions. The establishment of the foreign exchange interbank market has provided a platform for a more efficient allocation of foreign currency among banks. To date, institutional factors coupled with market conditions, notably a surplus of foreign exchange in the system, have held back the efficient functioning of the interbank market. Therefore, the CBS will continue to work closely with banks, and make use of technical assistance from the IMF, to improve the efficiency of the interbank foreign exchange market.

\section{Financial sector supervision and development}

\section{The CBS will continue to strengthen the supervision and regulation of the}

financial sector. The decision of the government to transfer supervision of state-owned nonbank financial institutions to the CBS in late 2009 will allow for a more effective oversight of these institutions. During 2010, we will continue to implement our 18-month action plan for strengthening risk-based supervision. We intend to increase the minimum paid-up capital requirement for holders of a domestic banking license and will promote the use of the net tangible capital ratio as an added measure of capital adequacy. We will seek technical assistance from the IMF to further refine prudential regulations and build domestic supervisory capacity. One of our key objectives is to enhance trust of the public in the banking system and to this end we are considering the possibility of introducing a deposit insurance scheme in 2011/12 and will study its feasibility and optimal operational modalities. 
We also intend to develop guidelines for a crisis preparedness program for the banking system that could be extended to nonbank financial institutions and other financial intermediaries as warranted.

43. We will intensify our efforts to promote transparency in our offshore financial sector through enhanced coordination between the Seychelles International Business Authority, which supervises the offshore sector, the CBS, and the Financial Intelligence Unit. Furthermore, we will continue to co-operate with international bodies like OECD and the FATF and have sought technical assistance from the Fund for a diagnostic assessment of the offshore sector.

44. The CBS will continue to modernize the national payment system. Several important steps were taken during 2009, including the establishment of a complete requirements and standards document for electronic funds transfers that replaced the manual settlement system. We intend to introduce an Electronic Clearing House, a National Payment System; a National Clearing and Settlement System Bill; promote the use of plastic cards; and implement a real time gross settlement system in 2010/11.

\section{CBS governance and capitalization}

45. The CBS will press forward with its program of improving internal control and audit systems. An updated safeguards assessment is required for the EFF. Building on the measures taken in response to the first safeguards assessment, the CBS will implement the recommendations of the updated assessment in 2010-11.

46. To improve the efficiency of its operations and in line with the modernization of the banking services activities, the CBS is working to introduce a core banking system in 2011. A complete needs analysis and requirements definition for the CBS Core Banking System has been completed with the assistance of the IMF's Monetary and Capital Markets Department and our external auditors.

47. The CBS is undercapitalized, which if left unaddressed, could constrain its ability to implement prudent monetary policies. As prescribed by the CBS Act, our intention was to gradually increase the central bank's capital base through the retention of 50 percent of annual profits until the target level of 10 percent of monetary liabilities is reached (up from 4 percent in 2008). However, significant valuation losses, due to the significant appreciation of the rupee, has eroded CBS's operating profits. If the external auditors determine that capital of the CBS is further impaired following closure of the 2009 annual accounts in early 2010, the government will promptly fully replenish the CBS' capital base, as required by the law, through a direct securitized injection. This would strengthen the balance sheet of the CBS and enhance its portfolio of tradable securities to be used in open market operations. 


\begin{tabular}{|ll|l|}
\hline \multicolumn{2}{|l|}{ Action } & Timing \\
\hline$\bullet$ & Complete CBS procedures manual & December 2009 \\
\hline$\bullet$ & Publish commercial bank supervision report & December 2009 \\
\hline$\bullet$ & Gradually raise minimum capital requirements for domestic banks. & $2010-11$ \\
\hline$\bullet$ & $\begin{array}{l}\text { Progressively raise capitalization of CBS to full level of 10 percent of } \\
\text { monetary liabilities }\end{array}$ & $2010-12$ \\
\hline$\bullet$ & $\begin{array}{l}\text { Introduce an integrated IT-based accounting framework at CBS (core } \\
\text { banking system). }\end{array}$ & September 2010 \\
\hline$\bullet$ & Adopt IFRS for 2009 CBS accounts & March 2010 \\
\hline$\bullet$ & $\begin{array}{l}\text { Present a National Electronic Clearing and Settlement System Bill to the } \\
\text { National Assembly }\end{array}$ & June 2010 \\
\hline$\bullet$ & Implementation of the real time gross settlement system. & 2010-11 \\
\hline$\bullet$ & Introduce a business continuity plan at CBS & September 2010 \\
\hline$\bullet$ & Adopt a crisis preparedness plan for financial system & December 2010 \\
\hline$\bullet$ & $\begin{array}{l}\text { Produce a financial survey encompassing both bank and nonbank financial } \\
\text { institutions }\end{array}$ & September 2010 \\
\hline$\bullet$ & $\begin{array}{l}\text { Introduce a new methodology for calculating application and annual license } \\
\text { fees for domestic and offshore banks }\end{array}$ & March 2010 \\
\hline
\end{tabular}

\section{F. National Statistics}

48. We are committed to improving the quality, periodicity, and timeliness of macroeconomic statistics. We are moving to address deficiencies in key macroeconomic data - especially GDP estimates, and external sector statistics - to improve their usefulness for economic analysis and policy formulation The Ministry of Employment will reinforce their efforts to produce high-frequency labor market data. We are also strengthening the National Statistics Bureau (NSB) and will implement recommendations of the management audit by end-2009. We have requested technical assistance from the IMF Statistics Department (on fiscal accounts) and from the World Bank (to strengthen the NSB). We will bring Seychelles into line with international best practice, by aiming to implement the IMF's Special Data Dissemination Standard (SDDS) and the Balance of Payments and International Investment Position Manual 6. We will set up a central coordination unit at the NSB that acts as a databank and a hub for data distribution, and monitors the timeliness of submission of the data by various agencies. 


\begin{tabular}{|c|c|}
\hline Action & Timing \\
\hline - $\quad$ Adopt a strategic plan to upgrade national statistics & April 2010 \\
\hline $\begin{array}{l}\text { - Implement Balance of Payments and International Investment Position } \\
\text { Manual } 6\end{array}$ & 2011 \\
\hline - $\quad$ Publish General government fiscal statistics & April 2010 \\
\hline - $\quad$ Implement SDDS & 2011 \\
\hline
\end{tabular}

\section{Program Financing}

49. For the remainder of $\mathbf{2 0 0 9}$, the external nonproject financing requirement will be covered by disbursements from the IMF under the SBA (25 percent of quota; SDR 2.2 million) and under the prospective EFF (10 percent of quota; SDR 0.88 million); from the World Bank and African Development Bank development policy loans (US\$19 million); from the EU Vulnerability Flex (€8 million); and from external debt relief (US\$83 million, including on the US\$55 million in arrears accumulated in the first three quarters). For 2010, a financing gap of US\$68 million is expected to be closed by debt relief under the second tranche of the Paris Club agreement; the restructuring of the remainder of public external debt, notably with commercial creditors and non-Paris Club creditors; and disbursements under the EFF. For 2011-12, the financing gaps will be closed by external debt relief and disbursements under the EFF. We have also assumed a follow-up operation by the World Bank at the conclusion of the current Development Policy Loan.

\section{Program MONitoring}

\section{The EFF-supported program will be monitored by semi-annual program} reviews, with quarterly quantitative performance criteria and disbursements. The quarterly quantitative performance criteria (QPCs) for end 2009 and for 2010 are shown in Table 4. The structural benchmarks for end-2009 and 2010 are shown in Table 3 . The non zero ceilings on the contracting or guaranteeing of external debt are to allow for budgeted public project finance and program support from multilateral institutions exclusively.

51. The completion of the first review under the EFF will be based on meeting the QPCs at end-March 2010. The first review will focus on further strengthening operations at the CBS, reinforcing public financial management, bolstering the financial system, progress on normalizing relations with external creditors, implementing the tax reform, and the stance of fiscal policy. The first program review is expected to be completed by late June 2010. The second program review will be based on meeting the end-September 2010 QPCs and is scheduled to be completed by end-December 2010. It will focus on the 2011 Budget, progress in implementing the tax reform, and in strengthening public financial management. Quarterly financing assurance reviews will continue as long as public debt arrears remain outstanding. 
52. The attached Technical Memorandum of Understanding (TMU) defines the quantitative performance criteria, indicative targets and adjusters under the program. Seychelles will avoid introducing new exchange restrictions, multiple currency practices, or bilateral payments agreements in contradiction with Article VIII of the IMF's Articles of Agreement and imposing any import restrictions for balance of payments reasons. We stand ready to adopt any additional measures, in consultation with IMF staff, which may become necessary to ensure program success. 
Table 1. Seychelles: Quantitative Performance Criteria Under the Stand-By Arrangement, 2009

(Millions of Seychelles rupees; end-of-period)

\begin{tabular}{|c|c|c|c|c|c|c|c|c|c|c|c|c|}
\hline & \multicolumn{12}{|c|}{2009} \\
\hline & \multicolumn{3}{|c|}{ March } & \multicolumn{3}{|c|}{ June } & \multicolumn{3}{|c|}{ September } & \multicolumn{3}{|c|}{ December } \\
\hline & $\mathrm{EBS} / 09 / 32$ & Adjusted & Actual & EBS/09/92 & Adjusted & Actual & EBS/09/92 & Adjusted & Prel. & EBS/09/92 & Adjusted & Projected \\
\hline \multicolumn{13}{|l|}{$\begin{array}{l}\text { Performance criteria } \\
\end{array}$} \\
\hline Net international reserves of the CBS, millions of U.S. dollars (floor) ${ }^{1}$ & 20 & 25 & 76 & 70 & 66 & 81 & 83 & 60 & 117 & 94 & 85 & 130 \\
\hline Reserve money (ceiling) & 1,146 & & 1,051 & 1,266 & & 1,119 & 1,311 & & 1,169 & 1,351 & & 1,480 \\
\hline Primary balance of the consolidated government (cumulative floor) ) $^{2,3,4}$ & 73 & 173 & 678 & 858 & 849 & 995 & 1,006 & 1,022 & 1,381 & 1,173 & 1,302 & 1,375 \\
\hline $\begin{array}{l}\text { The contracting or guaranteeing of new external debt by the public sector } \\
\text { (Millions of U.S. dollars; cumulative ceiling) }{ }^{2}\end{array}$ & 10 & $\ldots$ & 0 & 10 & $\ldots$ & 0 & 34 & $\ldots$ & 0 & 39 & $\ldots$ & 24 \\
\hline $\begin{array}{l}\text { The contracting or guaranteeing of new short-term external debt by the public } s \\
\text { (Millions of U.S. dollars; cumulative ceiling) }{ }^{2}\end{array}$ & 0.0 & $\ldots$ & 0.0 & 0.0 & $\ldots$ & 0.0 & 0.0 & $\ldots$ & 0.0 & 0.0 & $\ldots$ & 0.0 \\
\hline The accumulation of external payments arrears by the public sector (ceiling) ${ }^{5}$ & 0.0 & $\ldots$ & 0.0 & 0.0 & $\ldots$ & 0.0 & 0.0 & $\ldots$ & 0.0 & 0.0 & $\ldots$ & 0.0 \\
\hline The accumulation of domestic payment arrears by the public sector (ceiling) & 0.0 & $\ldots$ & 0.0 & 0.0 & $\ldots$ & 0.0 & 0.0 & $\ldots$ & 0.0 & 0.0 & & 0.0 \\
\hline \multicolumn{13}{|l|}{ Memorandum items: } \\
\hline External non project financing (millions of U.S. dollars; cumulative) ${ }^{2}$ & -10.0 & -5.3 & -5.3 & -26.0 & $\ldots$ & -29.5 & -7.3 & $\ldots$ & -30.8 & -8.9 & $\ldots$ & -17.2 \\
\hline Program financing support ${ }^{2}$ & 0.0 & 0.0 & 0.0 & 0.0 & $\ldots$ & 0.0 & 19.0 & $\ldots$ & 0.0 & 19.0 & $\ldots$ & 19.6 \\
\hline Cash payments on foreign debt service ${ }^{2}$ & 10.0 & 5.3 & 5.3 & 26.7 & $\ldots$ & 29.5 & 28.4 & $\ldots$ & 30.8 & 30.4 & $\ldots$ & 48.1 \\
\hline External budget grants ${ }^{2}$ & & 0.0 & 0.0 & 0.6 & $\ldots$ & 0.0 & 2.1 & $\ldots$ & 0.0 & 2.5 & $\ldots$ & 11.3 \\
\hline Budget contingency $^{6}$ & 100 & $\ldots$ & 0.0 & 0.0 & $\ldots$ & 0.0 & 50 & $\ldots$ & 2.8 & 100 & $\ldots$ & 100 \\
\hline \multicolumn{13}{|l|}{ Program accounting exchange rates } \\
\hline SR/US\$ (end-of-quarter) & 16.00 & 16.00 & 16.09 & 14.65 & $\ldots$ & 14.65 & 14.65 & $\ldots$ & 14.65 & 14.65 & $\ldots$ & 14.65 \\
\hline US\$/Euro (end-of-quarter) & 1.46 & 1.46 & 1.33 & 1.33 & $\ldots$ & 1.33 & 1.33 & $\ldots$ & 1.33 & 1.33 & $\ldots$ & 1.33 \\
\hline US $\$ /$ UK pound (end-of-quarter) & 1.84 & 1.84 & 1.48 & 1.48 & $\ldots$ & 1.48 & 1.48 & $\ldots$ & 1.48 & 1.48 & $\ldots$ & 1.48 \\
\hline US\$/SDR (end-of-quarter) & 1.56 & 1.56 & 1.50 & 1.50 & $\ldots$ & 1.50 & 1.50 & $\ldots$ & 1.50 & 1.50 & $\ldots$ & 1.50 \\
\hline Reserve requirement (percent of deposits) & 13 & 13 & 13 & 13 & $\ldots$ & 12 & 12 & $\ldots$ & 12 & 12 & $\ldots$ & 12 \\
\hline
\end{tabular}

Sources: Seychelles authorities, and IMF staff estimates and projections.

${ }_{1}^{1}$ The floor will be adjusted downwards (upwards) for any shortfall (excess) in external nonproject financial support from that assumed in the program.

${ }^{2}$ Cumulative flows from the beginning of the calendar year.

${ }^{3}$ The floor will be adjusted upwards for any unused amounts of the contingency in the budget for the recapitalization of the banking sector assumed in the program

Amounts of the contingency in excess of programmed amounts need to be funded within the program limits.

${ }^{4}$ The floor on the primary fiscal surplus will be adjusted upwards (downwards) by any excess (shorffall) of external budget grants from that assumed in the program.

${ }_{5}^{5}$ The non-accumulation of new external payment arrears constitutes a continuous performance criterion. Excludes arrears for which a rescheduling agreement is sought.

Some external arrears were accumulated in December 2008, but cleared before end-December.

The floor on the primary surplus of the consolidated government will be adjusted upwards by the cumulative amount of the contingency for recapitalizing

the state-controlled financial institutions which is not used for that purpose. 
Table 2. Seychelles: Principal Reform Measures Implemented June-October 2009

Action

Timing

Fiscal policy

Adopted a tax policy strategy with FAD TA

June

Developed corporate governance guidelines for public entities

August

Adopted a single treasury account

September

Introduced legislation to mandate that all revenue collected by the SRC be

September posted to the Government's consolidated revenue account.

\section{Monetary policy}

Made operational new lending facilities to commercial banks

Introduced repo and reverse-repo facilities

Adopted modernized and strengthened Financial Institutions Act

Updated and strengthened credit concentration and connected lending regulations

Eliminated the local asset ratio

August

Initiated a measured reduction in reserve requirements

August

Improved liquidity forecasting and strengthened monetary targeting

Ongoing

\section{CBS governance and safeguards}

Launched a tender to upgrade CBS's IT system to introduce an integrated accounting framework

September

\section{Public enterprise reforms}

Completed external audits of the 2008 accounts of seven major public enterprises in accordance with international audit standards

Prepared a strategic assessment for each major public enterprise

June

June

June

June

Conducted management audits of major public enterprises

Adopted a Public Enterprise Monitoring and Control Act

Established a public enterprise monitoring division in Ministry of Finance

\section{Foreign exchange regime and reserves management}

Approved a modernized Foreign Exchange Act

Established a Foreign Reserves Management Committee at the CBS

Adopted foreign reserves management guidelines

September

September

Ongoing

September

September

\section{Financial sector reforms}

Adopted a modernized and strengthened Financial Institutions Act

June

In consultation with IMF technical experts:

- Introduced documentation on the acquisition of substantial interests

June

- Updated credit concentration regulations and connected lending

June

- Introduced CBS manual on prompt and corrective actions

September 
Table 3. Seychelles: Structural Benchmarks for 2009-10

\section{Measure}

- Introduce a treasury single account.

- Adopt foreign reserves management investment guidelines.

- Adopt Public Enterprise Monitoring and Control act.

- Complete CBS procedures manual (MEFP, \46).

- $\quad$ CBS to publish commercial bank supervision report (MEFP, \46).

- Amend the Business Tax Act in line with the tax reform strategy (MEFP, \22).

- Cabinet approval of customs reform strategy and implementation plan (MEFP, \23).

- Publish general government fiscal statistics (MEFP, П29).

- Submit to National Assembly a new customs management act (MEFP, \23).

- Introduce Personal Income Tax (MEFP, \22).

- Introduce budget submissions protocols and procedures (MEFP, $\llbracket 28$ ).

- $\quad$ Adopt a new chart of account for the 2011 budget (MEFP, |29).

- Submit to National Assembly a bill creating a national clearing house and settlement system (MEFP, ף44).
Target date

End-September 2009

End-September 2009

End-September 2009

End-December 2009

End-December 2009 In progress.

End-December 2009 In progress.

End-March 2010

End-April 2010

End-June 2010

July 1,2010

End-July 2010

End-November 2010

End-December 2010

\section{Status}

Met. The TSA is leveraging progress on PFM.

Met. The CBS Board adopted guidelines on September 25, 2009.

Met. Parliamentary approval secured on

September 28, 2009.

In progress. 
Table 4. Seychelles: Quantitative Performance Criteria Under the EFF, December 2009 - December 2010 (Millions of Seychelles rupees; end-of-period)

\begin{tabular}{|c|c|c|c|c|c|}
\hline & \multirow{3}{*}{$\begin{array}{c}2009 \\
\text { December } \\
\text { Prog. }\end{array}$} & \multicolumn{4}{|c|}{2010} \\
\hline & & \multirow{2}{*}{$\begin{array}{l}\text { March } \\
\text { Prog. }\end{array}$} & \multirow{2}{*}{$\begin{array}{l}\text { June } \\
\text { Prog. }\end{array}$} & \multirow{2}{*}{$\begin{array}{r}\text { September } \\
\text { Prog. }\end{array}$} & \multirow{2}{*}{$\begin{array}{r}\text { December } \\
\text { Prog. }\end{array}$} \\
\hline & & & & & \\
\hline \multicolumn{6}{|l|}{ Performance criteria } \\
\hline Net international reserves of the CBS, millions of U.S. dollars (floor) ${ }^{1}$ & 130 & 133 & 128 & 142 & 168 \\
\hline Reserve money (ceiling) & 1,480 & 1,537 & 1,592 & 1,676 & 1,753 \\
\hline Primary balance of the consolidated government (cumulative floor) ${ }^{2,3}$ & 1,375 & 261 & 437 & 659 & 761 \\
\hline $\begin{array}{l}\text { The contracting or guaranteeing of new external debt by the public sector } \\
\text { (Millions of U.S. dollars; cumulative ceiling) }{ }^{2}\end{array}$ & 39 & 2 & 3 & 13 & 37 \\
\hline $\begin{array}{l}\text { The contracting or guaranteeing of new short-term external debt by the public } \\
\text { (Millions of U.S. dollars; cumulative ceiling) }\end{array}$ & 0.0 & 0.0 & 0.0 & 0.0 & 0.0 \\
\hline The accumulation of external payments arrears by the public sector (ceiling) ${ }^{3}$ & 0.0 & 0.0 & 0.0 & 0.0 & 0.0 \\
\hline The accumulation of domestic payment arrears by the public sector (ceiling) & 0.0 & 0.0 & 0.0 & 0.0 & 0.0 \\
\hline \multicolumn{6}{|l|}{ Memorandum items: } \\
\hline External non project financing (millions of U.S. dollars; cumulative) ${ }^{2}$ & -17.2 & 1.7 & 1.8 & 9.0 & 31.8 \\
\hline Program financing support & 19.6 & 0.0 & 0.0 & 0.0 & 19.6 \\
\hline Cash payments on foreign debt service & 48.1 & 1.8 & 5.8 & 6.9 & 9.2 \\
\hline External budget grants & 11.3 & 3.5 & 7.6 & 15.9 & 21.4 \\
\hline \multicolumn{6}{|l|}{ Program accounting exchange rates } \\
\hline SR/US\$ (end-of-quarter) & 11.00 & 11.00 & 11.00 & 11.00 & 11.00 \\
\hline US\$/Euro (end-of-quarter) & 1.48 & 1.48 & 1.48 & 1.48 & 1.48 \\
\hline US $\$ /$ UK pound (end-of-quarter) & 1.65 & 1.65 & 1.65 & 1.65 & 1.65 \\
\hline US\$/SDR (end-of-quarter) & 1.59 & 1.59 & 1.59 & 1.59 & 1.59 \\
\hline
\end{tabular}

Sources: Seychelles authorities, and IMF staff estimates and projections.

${ }^{1}$ The floor will be adjusted downwards (upwards) for any shortfall (excess) in external nonproject financial support from that assumed in the program.

${ }^{2}$ Cumulative flows from the beginning of the calendar year.

${ }^{3}$ The non-accumulation of new external payment arrears constitutes a continuous performance criterion. Excludes arrears for which a rescheduling agreement is sought. 


\section{Attachment 2}

\section{SEyChelles: TeChNiCAL MEMORANDUM OF UNDERSTANDing}

1. This technical memorandum of understanding presents the definitions of variables included in the quantitative performance criteria and indicative targets set out in the memorandum of economic and financial policies (MEFP), the key assumptions, and the reporting requirements of the Government and the Central Bank of Seychelles (CBS) needed to adequately monitor economic and financial developments. The quantitative performance criteria and indicative targets, and the benchmarks for 2009-10 are listed in Tables 1 and 3 of the MEFP, respectively.

\section{Quantitative Performance Criteria}

\section{A. Net International Reserves of the CBS (Floor)}

\section{Definition}

2. Net international reserves (NIR) of the CBS are defined for program monitoring purposes as reserve assets of the CBS, minus reserve liabilities of the CBS (including liabilities to the IMF). Reserve assets of the CBS are claims on nonresidents that are readily available (i.e., liquid and marketable assets, free of any pledges or encumberments and excluding project balances and blocked or escrow accounts, and bank reserves in foreign currency maintained for the purpose of meeting the reserve requirements), controlled by the CBS, and held for the purpose of intervening in foreign exchange markets. They include holdings of SDRs, holdings of foreign exchange, demand and short-term deposits at foreign banks abroad, fixed-term deposits abroad that can be liquidated without penalty, and any holdings of investment-grade securities. Reserve liabilities of the CBS comprise liabilities to nonresidents contracted by the CBS, any net off-balance sheet position of the CBS (futures, forwards, swaps, or options) with either residents or nonresidents, including those to the IMF.

\section{Calculation method}

3. For program monitoring purposes, reserves assets and liabilities at each test date, must be converted into U.S. dollars using the end of period exchange rates assumed in the program.

\section{Monitoring and reporting}

4. At each program test date (through end-December 2009), the quarterly net international reserves data submitted by the CBS to the IMF will be audited by the CBS external auditors in accordance with International Standards on Auditing, to ensure conformity with the program definition and calculation methods. Subsequent to endDecember 2009, quarterly audits will be conducted by the internal audit division. Audit 
reports will be submitted to the CBS, with a copy to the IMF, no later than two months after each test date.

\section{Adjusters}

5. The floor on the CBS's NIR will be adjusted upward (downward) by the amount by which the external nonproject loans and cash grants exceeds (falls short of) the amounts assumed in the program (MEFP Table 1). The floors will also be adjusted upwards (downwards) by the amount that external debt service payments fall short (exceed) the amounts assumed in the program.

\section{B. Reserve Money (Ceiling)}

\section{Definition}

6. Reserve money is equivalent to currency issued and deposits held by financial institutions at the central bank (bank reserves), including those denominated in foreign currencies. Evaluation of performance of reserve money with respect to the program ceiling will be done at the program accounting exchange rate.

\section{Monitoring and reporting}

7. For each program test date (through end-December 2009), the quarterly reserve money data submitted by the CBS to the IMF will be audited by the CBS' external auditors in accordance with International Standards on Auditing, to ensure conformity with the program definition. Subsequent to end-December 2009, quarterly audits will be conducted by the internal audit division. Audit reports will be submitted to the CBS, with a copy to the IMF, no later than two months after each test date.

\section{Primary Balance of the Consolidated Government (Cumulative Floor)}

8. The consolidated government primary balance from above the line on a commitment basis is defined as total consolidated government and social security fund revenues (excluding privatization and long-term lease income receipts) less all noninterest (primary) expenditures of the government and social security fund.

\section{Public External Debt (Ceiling)}

9. The ceiling applies to the contracting or guaranteeing of new external liabilities by the public sector (including the central government, the CBS, and all public agencies and parastatals for operations that are not directly linked to commercial activities). The ceiling does not apply to the use of Fund resources, operations related to external debt restructuring; normal import related credits; purchases of treasury securities by nonresidents; or borrowing by parastatals in the conduct of normal commercial operations. The non zero ceilings on the contracting or guaranteeing of external debt are to allow for normal public project finance and program support from multilateral institutions exclusively. Debt shall be valued in 
U.S. dollars at program exchange rates. A zero subceiling on short-term external debt applies continuously to the contracting or guaranteeing of short-term external debt by the public sector, with an original maturity of up to and including one year.

10. For the purpose of this performance criterion, the term "debt" has the meaning set forth in point No. 9 of the Executive Board's Guidelines on Performance Criteria with Respect to Foreign Debt (Decision No. 12274-(00/85)). Debt is understood to mean a current, non contingent liability, created under a contractual arrangement through the provision of value in the form of assets (including currency) or services, and which requires the obligor to make one or more payments in the form of assets (including currency) or services, at some future points in time; these payments will discharge the principal and/or interest liabilities incurred under the contract. The ceiling on contracting official and officially guaranteed external debt includes all form of debt, including:

(i) loans, i.e., advances of money to the obligor by the lender made on the basis of an undertaking that the obligor will repay the funds in the future (including deposits, bonds, debentures, commercial loans, and buyers credits) and temporary exchanges of assets that are equivalent to fully collateralized loans under which the obligor is required to repay the funds, and usually pay interest, by repurchasing the collateral from the buyer in the future (such as repurchase agreements and official swap arrangements);

(ii) suppliers credits, i.e., contracts where the supplier permits the obligor to defer payments until some time after the date on which the goods are delivered or services are provided;

(iii) leases, i.e., arrangements under which property is provided which the lessee has the right to use for one or more specified period(s) of time that are usually shorter than the total expected service life of the property, while the leasor retains title to the property. The debt is the present value (at the inception of the lease) of all lease payments expected to be made during the period of the agreement excluding those payments that cover the operation, repair, or maintenance of the property; and

(iv) Arrears, penalties, and judicially awarded damages arising from the failure to make payment under a contractual obligation that constitutes debt are debt.

\section{E. External Arrears of the Public Sector}

11. The nonaccumulation of arrears to external creditors will be a continuous performance criterion under the program. External payments arrears for program monitoring purposes are defined as the amount of external debt service due and not paid within the contractually agreed period, including contractual and late interest. Arrears resulting from the nonpayment of debt service for which a clearance framework has been agreed or a rescheduling agreement is sought are excluded from this definition. 


\section{F. Budget Expenditure Arrears}

12. The nonaccumulation of budget expenditure arrears will be a continuous performance criterion under the program. Budget expenditure arrears are defined as the sum of (1) any invoice that has been received by a spending agency from a supplier of goods, services, and capital goods delivered and verified, and for which payment has not been made within the contractually agreed period, or in the absence of a grace period, within 30 days; (2) unpaid wages, pensions, or transfers, pending for longer than 30 days to domestic or foreign residents, irrespective of the currency denomination of the debt.

\section{DATA AND INFORMATION}

13. The Seychelles authorities (government and CBS) will provide Fund staff with the following data and information according to the schedule provided.

\section{The CBS will report}

Weekly (within one week from the end of the period)

- Reserve money.

- Foreign exchange reserves position.

- A summary table on the foreign exchange market transactions.

- The results of the liquidity deposit auctions, primary treasury bill auctions, and secondary auctions.

Monthly (within four weeks from the end of the month)

- The monetary survey in the standardized report form format.

- The foreign exchange cash flow, actual and updated.

- Financial soundness indicators.

- Stock of government securities in circulation by holder (banks and nonbanks) and by original maturity and the debt service profile report.

\section{The Ministry of Finance will report}

Monthly (within two weeks from the end of the month):

- Consolidated government operations on a commitment basis and cash basis in the IMF-supported program format.

- The detailed revenues and expenditures of the central government and social security fund.

- Monthly accounts of the public nonbank financial institutions. 
- Import and export data from the customs department.

- Monthly public debt report.

The government and CBS will consult with Fund staff on all economic and financial measures that would have an impact on program implementation, and will provide any additional relevant information as requested by Fund staff. 


\section{APPENDIX II}

\section{SEyChelles: DebT SuSTAINABILITY ANALYSIS}

This DSA confirms that public debt in Seychelles remains unsustainable without a restructuring of the stock of public external debt relief on terms comparable to that agreed with Paris Club creditors in April, which recognized Seychelles' limited repayment capacity. Discussions with all creditor groups have advanced well in recent months and Seychelles is preparing to launch an exchange offer to commercial creditors. This analysis suggests that, together with restructuring of remaining stock of public external debt on comparable terms to the PC agreement, and strong and sustained fiscal adjustment assumed under the program, sustainability is within reach. However, even with such a restructuring, bound tests indicate that Seychelles remains highly vulnerable to shocks.

\section{Macroeconomic assumptions}

The debt sustainability analysis is based on the macroeconomic framework presented in the present staff report:

- Near-term economic growth outlook has been revised upwards on account of the economy's favorable response to the reforms and improving global conditions, with real GDP expected to contract by 7.5 percent in 2009 . Growth is expected to recover to 4 percent in 2010, supported by resumption of FDI-financed projects and recovery of tourism, and stabilize at around 5 percent though 2015, before easing to 4-41/2 percent in the long term.

- Supported by tight fiscal and monetary policy, inflation has declined sharply and is expected to remain in the low single digits, aided by sharp declines in world food and fuel prices. It is assumed to remain at around 3 percent going forward in line with major trading partners' inflation.

- The near-term current account outlook has improved from that at the second review on account of higher grants and lower imports. With improved economic conditions, and early indications of a recovery in tourism earnings, the tourism forecasts for 2009 have been revised to a 12 percent drop, from the originally projected 22 percent decline, while tuna exports are expected decline by 8 percent, as the international community has responded to the piracy threat. Over the medium term, tourism growth is expected to recover, peaking at 13 percent in 2013-14 and moderating to a long-run steady rate of around 8 percent a year. Tuna exports are expected to revive by 3 percent in 2010 and 2 percent in 2011.

- Net FDI inflows are expected to decline sharply in 2009 to 25 percent of GDP, as a number of large hotel projects have been postponed or slowed. This reflects tighter credit conditions and the slow recovery of the tourism outlook. FDI flows are expected to recover in 2011 and rise sharply over the medium term before stabilizing at around 25 percent of GDP over the long run. 
- Official foreign exchange reserves are targeted to build up gradually to about 3.8 months of imports by 2019, a necessary recovery from the depleted levels of 2008.

\section{Evolution of the debt stock in 2009}

- Public external debt is estimated at US\$733 million or 95.7 percent of GDP at end-September 2009 (Table 1). About 67 percent of the debt was owed to commercial creditors, while the PC countries account for about 16 percent of the total. Seychelles has no arrears with multilateral creditors. At end-

September 2009 some 33 percent of the debt stock was in arrears (US\$239.2 million).

- In April 2009, PC creditors granted exceptional treatment to Seychelles under the Evian approach. ${ }^{1}$ US\$140 million of arrears were normalized. The restructuring

\begin{tabular}{crr}
\multicolumn{3}{c}{ Table 1. External Debt ${ }^{1}$} \\
\hline & End-2008 & 2009 Q3 \\
\hline External debt stock $^{2}$ & 767.1 & 733.2 \\
Multilateral & 52.2 & 27.9 \\
Bilateral & 239.0 & 212.8 \\
Paris Club & 139.8 & 115.6 \\
Non-Paris Club & 99.1 & 97.2 \\
Commercial & 475.9 & 492.6 \\
External debt arrears & 321.7 & 239.2 \\
Multilateral & 0.0 & 0.0 \\
Bilateral & 175.0 & 43.6 \\
Paris Club & 112.4 & 0.0 \\
Non-Paris Club & 62.6 & 43.6 \\
Commercial & 146.7 & 195.6 \\
\hline
\end{tabular}

' Preliminary and subject to reconciliation with creditors. Includes CBS external debt.

2 Includes arrears. of Seychelles debt involves both flow and stock rescheduling with the debt stock reduced by 45 percent in two tranches (July 2009 and mid-2010) and the remaining amounts rescheduled over 18 years including a five-year grace. No repayments will be made in 2009 other than a "goodwill" payment of US\$1 million made in June, with very limited payments due in the next few years as moratorium interest is partially deferred through 2012 (Table 2)

- A restructuring agreement with Malaysia, on Paris Club terms was signed in October, restructuring about $\$ 12$ million in overdue claims.

- The stock of public external debt is expected to rise to US\$755 million (99 percent of GDP) by end 2009, reflecting disbursements from multilateral agencies (some \$24 million).

\footnotetext{
${ }^{1}$ The agreement covered all the debt to Paris Club. South Africa, a significant non-Paris Club creditor, participated in the meeting and was a signatory of the minute.

2 The Paris Club agreement did not include a lease contracted by a parastatal; this will continue to be serviced by the parastatal.
} 
Table 2. External Debt Service ${ }^{1}$

\begin{tabular}{|c|c|c|c|c|c|c|}
\hline \multirow[b]{3}{*}{ Interest } & 2007 & 2008 & 2009 & 2010 & 2011 & 2012 \\
\hline & \multicolumn{6}{|c|}{ (In millions of US dollars) } \\
\hline & 32.7 & 32.7 & 31.8 & 31.8 & 31.4 & 11.2 \\
\hline Multilateral & 1.8 & 1.8 & 2.3 & 3.8 & 5.8 & 8.2 \\
\hline Bilateral & 3.5 & 3.5 & 4.4 & 5.3 & 4.3 & 3.0 \\
\hline Paris Club ${ }^{2}$ & 1.4 & 1.4 & 1.1 & 1.8 & 1.5 & 1.3 \\
\hline Non-Paris Club ${ }^{2}$ & 2.1 & 2.1 & 3.2 & 3.5 & 2.7 & 1.7 \\
\hline Commercial & 27.4 & 27.5 & 25.1 & 22.7 & 21.3 & 0.0 \\
\hline Amortization & 66.9 & 188.7 & 81.5 & 58.2 & 256.4 & 10.5 \\
\hline Multilateral & 4.7 & 4.7 & 25.1 & 3.7 & 3.5 & 2.0 \\
\hline Bilateral & 24.5 & 23.6 & 11.3 & 15.7 & 9.4 & 7.0 \\
\hline Paris Club & 4.7 & 3.9 & 2.0 & 3.9 & 2.0 & 2.0 \\
\hline Non-Paris Club & 19.7 & 19.7 & 9.3 & 11.8 & 7.5 & 4.9 \\
\hline Commercial & 37.7 & 160.4 & 45.2 & 38.7 & 243.4 & 1.5 \\
\hline \multicolumn{7}{|l|}{ Memorandam items } \\
\hline Deferred interest ${ }^{3}$ & & & 4.4 & 1.0 & 1.0 & 1.0 \\
\hline
\end{tabular}

1/ Debt service due, after restructuring agreements with Paris Club, South Africa and Malaysia. Both interest and amortization include IMF.

2/ After deferral of moratorium interest by Paris Club, South Africa and Malaysia.

\section{Baseline scenario}

The baseline scenario incorporates restructuring agreements reached to date, notably that with Paris Club creditors, South Africa, and Malaysia. ${ }^{3}$ External debt levels rise initially at end 2009 to about 151 percent of GDP as arrears are financed, before falling in 2010 as the existing agreements are fully implemented (Tables 3 and $3 \mathrm{a}$ and Figure 1). However, the debt dynamics become quickly unsustainable in the absence of agreements with remaining creditors on the majority of the debt. This scenario assumes that the nonrestructured debt (including arrears) is serviced and that the financing gaps are closed, as assumed in earlier analyses, by a flow rescheduling on market terms at LIBOR plus 1300 basis points and with a three-year maturity. ${ }^{4}$ By 2019 the public external debt ratio rises steadily to about 404 percent of GDP. This explosive path results notwithstanding continued strong fiscal adjustment with a very similar profile for overall public debt (Table $3 \mathrm{~b}$ and Figure 1a). Furthermore, the bound tests highlight that the external debt is extremely sensitive to the underlying assumptions, with even moderate shocks triggering dramatic explosive dynamics.

\footnotetext{
${ }^{3}$ Assumes the second tranche of Paris Club creditors relief becomes effective as expected in mid-2010.

${ }^{4}$ The assumed risk premium is marginally above the highest levels of EMBI spreads on sovereign external debt of other highly indebted countries with performing debt.
} 


\section{Alternative scenario ${ }^{5}$}

This scenario assumes that public external debt at end-2008 is restructured on comparable cash flow terms with the PC agreement. Under these assumptions, public external debt would decline to 60 percent by end 2010 and gradually further throughout the period to about 44 percent of GDP in 2019 (Tables 4 and 4a, Figure 2). Total public debt, would follow a similar profile declining to 97 percent in 2010 and easing further to just under 50 percent of GDP by 2019. This path suggests a sustainability profile is achieved, although the standardized bound tests show that Seychelles remains very vulnerable to a variety of shocks (Table 4b, and Figure 2a).

\footnotetext{
${ }^{5}$ This hypothetical scenario is included for illustrative purposes only. It is based on technical assumptions regarding the restructuring of debts to all other non-Paris Club creditors on terms comparable to those agreed with Paris Club creditors, without pre-judging the terms of such a restructuring.
} 
Table 3. Seychelles: Balance of Payments Baseline Scenario (Paris Club Restructuring only and Nonconcessional Refinancing), 2008-19 (Millions of U.S. dollars)

\begin{tabular}{|c|c|c|c|c|c|c|c|c|c|c|c|c|}
\hline & 2008 & 2009 & 2010 & 2011 & 2012 & 2013 & 2014 & 2015 & 2016 & 2017 & 2018 & 2019 \\
\hline & Prel. & Proj. & Proj. & & & & & & & & & \\
\hline $\begin{array}{l}\text { Current account } \\
\text { (Percent of GDP) }\end{array}$ & $\begin{array}{r}-412 \\
-45\end{array}$ & $\begin{array}{r}-227 \\
-30\end{array}$ & $\begin{array}{r}-421 \\
-43\end{array}$ & $\begin{array}{r}-520 \\
-52\end{array}$ & $\begin{array}{r}-610 \\
-57\end{array}$ & $\begin{array}{r}-661 \\
-59\end{array}$ & $\begin{array}{r}-767 \\
-65\end{array}$ & $\begin{array}{r}-832 \\
-66\end{array}$ & $\begin{array}{r}-937 \\
-70\end{array}$ & $\begin{array}{r}-1,103 \\
-77\end{array}$ & $\begin{array}{r}-1,273 \\
-84\end{array}$ & $\begin{array}{r}-1,481 \\
-91\end{array}$ \\
\hline Trade balance & -379 & -130 & -263 & -235 & -252 & -207 & -202 & -151 & -118 & -96 & -65 & -33 \\
\hline Income, net & -103 & -156 & -202 & -338 & -412 & -507 & -620 & -737 & -876 & $-1,046$ & $-1,246$ & $-1,484$ \\
\hline Of which: interest payments due ${ }^{1}$ & -33 & -83 & -117 & -212 & -267 & -339 & -426 & -519 & -639 & -798 & -998 & $-1,241$ \\
\hline Current transfers, net & 70 & 58 & 44 & 53 & 53 & 54 & 55 & 56 & 56 & 39 & 39 & 37 \\
\hline Capital and financial account & 119 & 431 & 460 & 570 & 666 & 721 & 823 & 873 & 977 & 1,143 & 1,323 & 1,531 \\
\hline Capital account & 5 & 53 & 62 & 17 & 17 & 10 & 15 & 17 & 17 & 17 & 17 & 16 \\
\hline Financial account & 113 & 378 & 398 & 553 & 649 & 711 & 808 & 856 & 960 & 1,126 & 1,306 & 1,515 \\
\hline Direct investment, net & 350 & 192 & 245 & 258 & 295 & 318 & 383 & 374 & 364 & 366 & 367 & 369 \\
\hline Portfolio investment, net & 1 & 1 & 1 & 1 & 1 & 5 & 8 & 11 & 17 & 25 & 38 & 38 \\
\hline Other investment, net ${ }^{2}$ & -238 & 185 & 152 & 294 & 353 & 388 & 417 & 471 & 579 & 735 & 901 & 1,108 \\
\hline Net errors and omissions & 82 & 56 & 0 & 0 & 0 & 0 & 0 & 0 & 0 & 0 & 0 & 0 \\
\hline Overall balance & -212 & 259 & 39 & 50 & 56 & 61 & 56 & 41 & 40 & 40 & 50 & 50 \\
\hline Financing & 212 & -260 & -39 & -50 & -56 & -61 & -56 & -41 & -40 & -40 & -50 & -50 \\
\hline Change in net international reserves (increase: - ) & 38 & -95 & -39 & -50 & -56 & -61 & -56 & -41 & -40 & -40 & -50 & -50 \\
\hline Change in gross official reserves (increase: - ) & 28 & -102 & -49 & -50 & -50 & -50 & -50 & -40 & -40 & -40 & -50 & -50 \\
\hline Prospective liabilities to IMF, net & 10 & 8 & 10 & 0 & -6 & -11 & -6 & -1 & 0 & 0 & 0 & 0 \\
\hline Other NFA (increase: -) & 0 & -24 & 0 & 0 & 0 & 0 & 0 & 0 & 0 & 0 & 0 & 0 \\
\hline Exceptional financing & 174 & -141 & 0 & 0 & 0 & 0 & 0 & 0 & 0 & 0 & 0 & 0 \\
\hline Change in arrears (increase: + ) 3,4 & 174 & -328 & 0 & 0 & 0 & 0 & 0 & 0 & 0 & 0 & 0 & 0 \\
\hline Clearance of arrears & & 187 & 0 & 0 & 0 & 0 & 0 & 0 & 0 & 0 & 0 & 0 \\
\hline Financing gap ${ }^{5}$ & 0 & 0 & 0 & 0 & 0 & 0 & 0 & 0 & 0 & 0 & 0 & 0 \\
\hline Financing gap (before closing) & 0 & 261 & 258 & 665 & 704 & 866 & 1,126 & 1,336 & 1,674 & 2,130 & 2,660 & 3,308 \\
\hline \multicolumn{13}{|l|}{ Memorandum items: } \\
\hline Gross international reserves (stock, e.o.p.) ${ }^{6}$ & 51 & 153 & 202 & 252 & 302 & 352 & 402 & 442 & 482 & 522 & 572 & 622 \\
\hline Months of prospective imports of goods and services & 1 & 1 & 2 & 2 & 3 & 3 & 3 & 3 & 3 & 3 & 4 & 4 \\
\hline Public external debt 1,4,6 & 767 & 1,159 & 1,285 & 1,561 & 1,899 & 2,244 & 2,630 & 3,080 & 3,658 & 4,420 & 5,377 & 6,539 \\
\hline (Percent of GDP) & 83 & 151 & 131 & 155 & 178 & 200 & 222 & 244 & 272 & 309 & 353 & 404 \\
\hline Of which: arrears ${ }^{1,3,4}$ & 322 & 0 & 0 & 0 & 0 & 0 & 0 & 0 & 0 & 0 & 0 & 0 \\
\hline (Percent of GDP) & 35 & 0 & 0 & 0 & 0 & 0 & 0 & 0 & 0 & 0 & 0 & 0 \\
\hline GDP & 921 & 766 & 982 & 1,009 & 1,070 & 1,119 & 1,186 & 1,262 & 1,343 & 1,430 & 1,522 & 1,620 \\
\hline
\end{tabular}

${ }^{1}$ Projections are based on identified new borrowing and rescheduled Paris Club debt. It assumes comparable cash flow treatment for two bilateral creditors that also signed the ${ }^{1}$ Projections are based on identified new borrowing and rescheduled Paris Club debt. It assumes comparable cash flow treatment for
agreement, and no debt restructuring for the remaining creditors. Arrears restructuring leads to higher rescheduled interest payments.

${ }^{2}$ Based on Paris Club agreement, the payments of principal and most interest during

2008 and through June 2009 are deferred.

3 In 2008, includes accelerated amortizing notes.

${ }^{4}$ Debt forgivenesss reflects: (i) the first stage of debt reduction (about 22.5) percent in July 2009; (ii) the second stage reduction (about 22.5 percent) in July 2010 on Paris Club debt and one bilateral creditor.

${ }^{5}$ Financing gaps are assumed to be closed by a hypothetical 3-year maturity amortizing loan at libor plus 13 percent.

${ }^{6}$ Starting with 2007, refers to CBS gross international reserves net of blocked deposits and project accounts. 


\begin{tabular}{|c|c|c|c|c|c|c|c|c|c|c|c|c|}
\hline & \multicolumn{11}{|c|}{ Projections } & \multirow[b]{2}{*}{2019} \\
\hline & 2008 & 2009 & 2010 & 2011 & 2012 & 2013 & 2014 & 2015 & 2016 & 2017 & 2018 & \\
\hline \multicolumn{13}{|c|}{ I. Baseline Projections } \\
\hline External debt 1/ & 83.3 & 151.3 & 130.9 & 154.7 & 177.6 & 200.5 & 221.9 & 244.1 & 272.3 & 309.1 & 353.3 & 403.6 \\
\hline Change in external debt & 14.1 & 68.0 & -20.4 & 23.8 & 22.9 & 22.9 & 21.4 & 22.2 & 28.2 & 36.8 & 44.2 & 50.3 \\
\hline Identified external debt-creating flows $(4+8+9)$ & 14.6 & 21.6 & -15.3 & 26.0 & 26.0 & 28.5 & 27.6 & 24.0 & 28.7 & 36.6 & 43.0 & 48.9 \\
\hline Current account deficit, excluding interest payments & 42.5 & 18.3 & 30.8 & 30.4 & 32.0 & 28.7 & 28.7 & 24.8 & 22.2 & 21.3 & 18.1 & 14.8 \\
\hline Deficit in balance of goods and services & 41.2 & 16.9 & 26.8 & 23.3 & 23.5 & 18.5 & 17.0 & 12.0 & 8.8 & 6.7 & 4.3 & 2.1 \\
\hline Exports & 110.5 & 116.7 & 102.1 & 104.2 & 105.7 & 109.5 & 112.8 & 113.1 & 113.6 & 114.1 & 114.0 & 114.2 \\
\hline Imports & 151.7 & 133.7 & 128.8 & 127.5 & 129.2 & 128.0 & 129.9 & 125.1 & 122.4 & 120.8 & 118.3 & 116.2 \\
\hline Net non-debt creating capital inflows (negative) & -38.0 & -25.0 & -24.9 & -25.6 & -27.6 & -28.4 & -32.3 & -29.7 & -27.1 & -25.6 & -24.1 & -22.8 \\
\hline Automatic debt dynamics $2 /$ & 10.1 & 28.2 & -21.1 & 21.2 & 21.6 & 28.2 & 31.3 & 28.9 & 33.6 & 40.8 & 49.0 & 56.9 \\
\hline Contribution from nominal interest rate & 2.2 & 11.4 & 12.0 & 21.6 & 25.8 & 31.2 & 37.0 & 41.3 & 47.7 & 56.1 & 66.2 & 77.4 \\
\hline Contribution from real GDP growth & 0.7 & 7.6 & -4.7 & -6.5 & -7.5 & -8.7 & -9.7 & -10.5 & -10.3 & -11.6 & -11.7 & -13.4 \\
\hline Contribution from price and exchange rate changes $3 /$ & 7.2 & 9.3 & -28.4 & 6.1 & 3.3 & 5.8 & 4.0 & -2.0 & -3.8 & -3.7 & -5.4 & -7.1 \\
\hline Residual, incl. change in gross foreign assets $(2-3)$ & -0.6 & 46.4 & -5.1 & -2.2 & -3.1 & -5.6 & -6.3 & -1.8 & -0.4 & 0.2 & 1.1 & 1.4 \\
\hline External debt-to-exports ratio (in percent) & 75.3 & 129.6 & 128.3 & 148.5 & 168.1 & 183.1 & 196.6 & 215.7 & 239.7 & 270.8 & 309.9 & 353.5 \\
\hline Gross external financing need (in billions of US dollars) 4 / & 0.6 & 0.3 & 0.6 & 0.9 & 1.0 & 1.2 & 1.5 & 1.7 & 2.1 & 2.5 & 3.0 & 3.6 \\
\hline in percent of GDP & 65.2 & 40.3 & 57.4 & 94.0 & 94.9 & 108.4 & 128.4 & 137.7 & 152.9 & 174.2 & 196.9 & 225.1 \\
\hline \multicolumn{13}{|l|}{ Key Macroeconomic Assumptions } \\
\hline Nominal GDP (US dollars) & 0.9 & 0.8 & 1.0 & 1.0 & 1.1 & 1.1 & 1.2 & 1.3 & 1.3 & 1.4 & 1.5 & 1.6 \\
\hline Real GDP growth (in percent) & -0.9 & -7.6 & 4.0 & 5.0 & 5.0 & 5.0 & 5.0 & 5.0 & 4.5 & 4.5 & 4.0 & 4.0 \\
\hline Exchange rate appreciation (US dollar value of local currency, change in percent) & -29.5 & -30.0 & 20.5 & -4.4 & -2.1 & -3.1 & -2.0 & -1.5 & -1.5 & -1.5 & -1.5 & -1.5 \\
\hline GDP deflator in US dollars (change in percent) & -9.4 & -10.1 & 23.1 & -4.4 & -2.1 & -3.1 & -2.0 & 0.9 & 1.6 & 1.4 & 1.4 & 1.4 \\
\hline Nominal external interest rate (in percent) & 2.9 & 11.4 & 10.2 & 16.6 & 17.2 & 17.9 & 19.0 & 19.7 & 20.8 & 21.8 & 22.6 & 23.1 \\
\hline Growth of exports (US dollar terms, in percent) & 16.6 & -12.2 & 12.1 & 4.9 & 7.5 & 8.4 & 9.2 & 6.7 & 6.9 & 6.9 & 6.3 & 6.6 \\
\hline Growth of imports (US dollar terms, in percent) & 33.4 & -26.7 & 23.5 & 1.7 & 7.4 & 3.7 & 7.5 & 2.6 & 4.1 & 5.1 & 4.2 & 4.6 \\
\hline Current account balance, excluding interest payments & -42.5 & -18.3 & -30.8 & -30.4 & -32.0 & -28.7 & -28.7 & -24.8 & -22.2 & -21.3 & -18.1 & -14.8 \\
\hline Net non-debt creating capital inflows & 38.0 & 25.0 & 24.9 & 25.6 & 27.6 & 28.4 & 32.3 & 29.7 & 27.1 & 25.6 & 24.1 & 22.8 \\
\hline \multicolumn{13}{|c|}{ II. Stress Tests for External Debt Ratio } \\
\hline A. Alternative Scenarios & & & & & & & & & & & & \\
\hline A1. Key variables are at their historical averages in 2009-2014 5/ & 83.3 & 122.9 & 120.5 & 121.0 & 120.6 & 117.9 & 114.5 & 115.4 & 117.6 & 120.4 & 123.7 & 126.9 \\
\hline \multicolumn{13}{|l|}{ B. Bound Tests } \\
\hline B1. Nominal interest rate is at baseline plus one standard deviation & 83.3 & 153.8 & 136.2 & 164.4 & 192.7 & 222.8 & 253.3 & 285.7 & 326.6 & 379.4 & 444.0 & 519.6 \\
\hline B2. Real GDP growth is at baseline minus two standard deviations & 83.3 & 167.0 & 152.3 & 186.8 & 218.0 & 246.0 & 263.5 & 281.9 & 307.1 & 340.6 & 382.5 & 431.5 \\
\hline B3. Non-interest current account is at baseline minus one standard deviations & 83.3 & 163.2 & 153.2 & 192.6 & 232.7 & 276.3 & 321.5 & 368.6 & 426.0 & 497.8 & 583.9 & 683.3 \\
\hline B4. Combination of B1-B3 using $1 / 4$ standard deviation shocks & 83.3 & 156.7 & 140.2 & 169.6 & 197.5 & 225.2 & 250.1 & 276.9 & 311.4 & 356.6 & 411.8 & 476.0 \\
\hline B5. One time 15 percent of additional real depreciation in 2009 & 83.3 & 183.1 & 151.0 & 170.6 & 186.2 & 198.6 & 204.8 & 212.4 & 225.4 & 244.9 & 269.3 & 296.9 \\
\hline
\end{tabular}

$1 /$ Includes CBS external debt.

2/ Derived as $[\mathrm{r}-\mathrm{g}-\rho(1+\mathrm{g})+\varepsilon \alpha(1+\mathrm{r})] /(1+\mathrm{g}+\rho+\mathrm{g} \rho)$ times previous period debt stock, with $\mathrm{r}=$ nominal effective interest rate on external debt; $\rho=$ change in domestic GDP deflator in US dollar terms,

$\mathrm{g}=$ real GDP growth rate, $\mathrm{e}=$ nominal appreciation (increase in dollar value of domestic currency), and $\mathrm{a}=$ share of domestic-currency denominated debt in total external debt.

$3 /$ The contribution from price and exchange rate changes is defined as $[-\rho(1+g)+\varepsilon \alpha(1+\mathrm{r})] /(1+\mathrm{g}+\rho+g \rho)$ times previous period debt stock. $\rho$ increases with an appreciating domestic currency $(\varepsilon>0)$

and rising inflation (based on GDP deflator).

4/ Defined as current account deficit, plus amortization on medium- and long-term debt, plus short-term debt at end of previous period.

5/ The key variables include real GDP growth; nominal interest rate; dollar deflator growth; and both non-interest current account and non-debt inflows in percent of GDP. 
Figure 1. External DSA: Bound Tests for Baseline Scenario (PC Restructuring and Nonconcessional Refinancing) ${ }^{1}$ (External debt in percent of GDP)

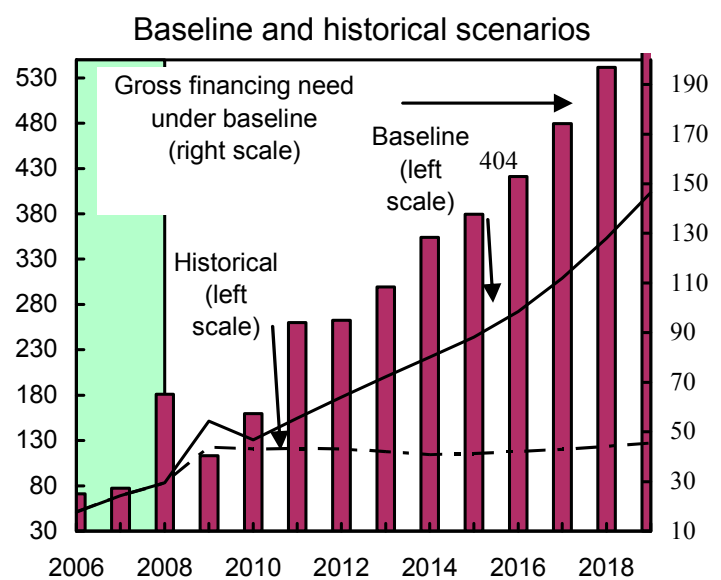

Growth shock

(percent per year)

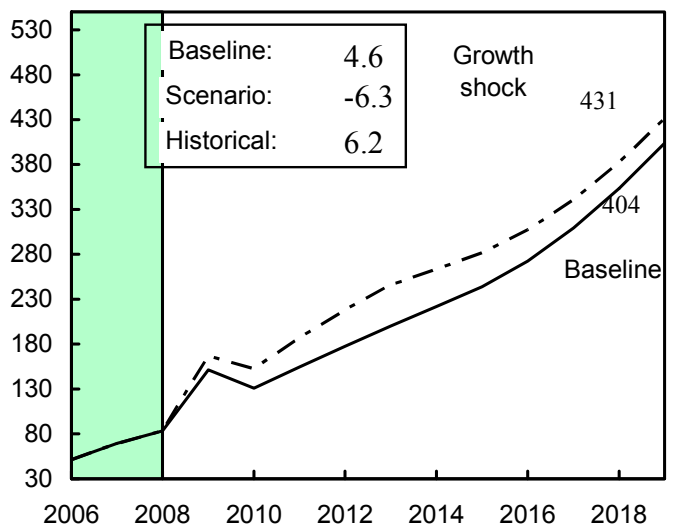

Combined shock $^{2}$

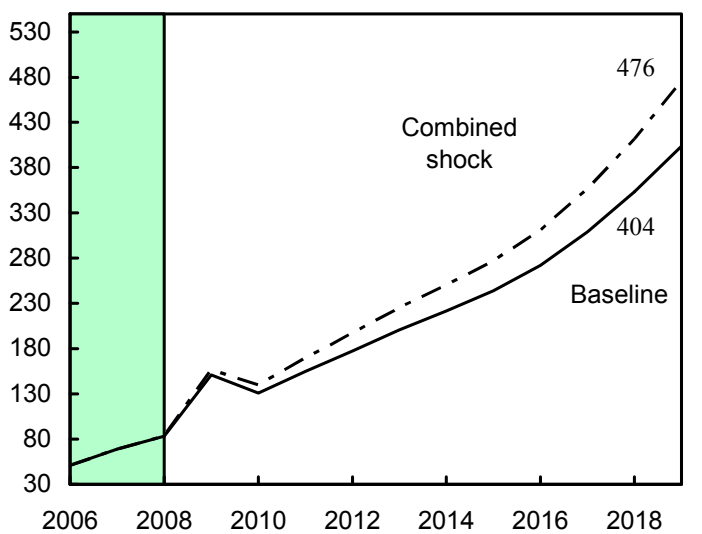

Interest rate shock (percent)

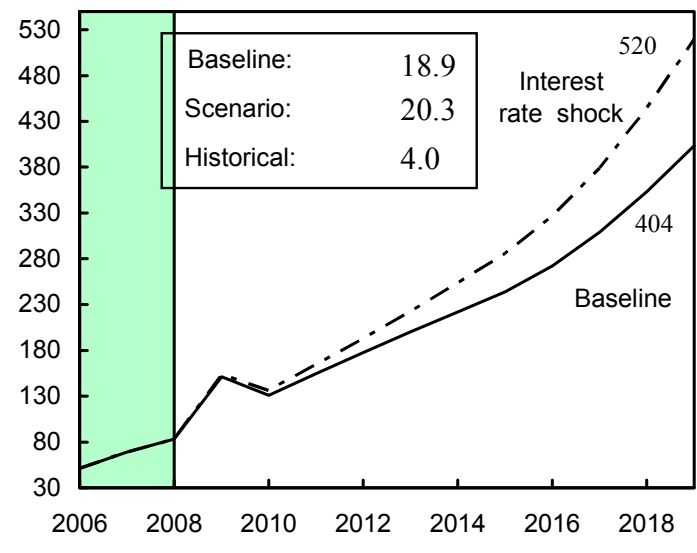

Non-interest current account balance shock ( percent of GDP)

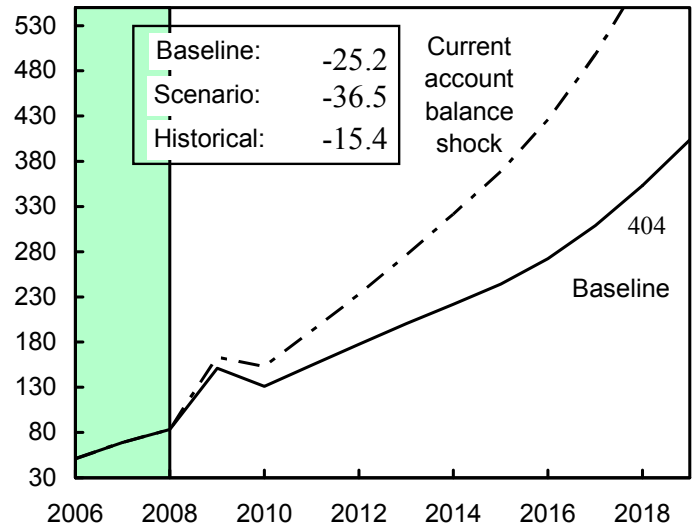

Real depreciation shock ${ }^{3}$

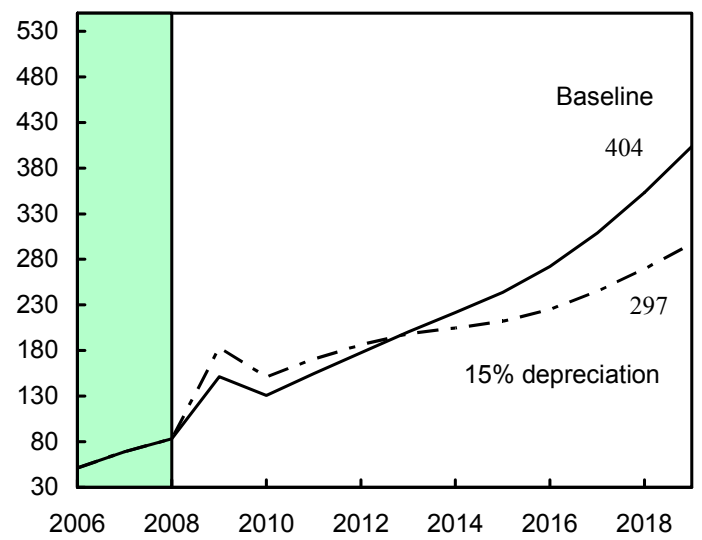

Sources: International Monetary Fund, Country desk data, and staff estimates.

${ }^{1}$ External financing gap is assumed to be closed by external borrowing at LIBOR plus $1300 \mathrm{bps}$, with three year maturity. Shaded areas represent actual data. Individual shocks are permanent one standard deviation shocks (two standard deviations for growth shock). Figures in the boxes represent average projections for the respective variables in the baseline and scenario being presented. Ten-year historical average for the variable is also shown.

${ }^{2}$ Permanent $1 / 4$ standard deviation shocks applied to real interest rate, growth rate, and current account balance.

${ }^{3}$ One-time real depreciation of additional 15 percent occurs in 2009. 
Table 3b. Seychelles: Public Sector Debt Sustainability Framework for Baseline Scenario (PC Restructuring only and Nonconcessional Refinancing), 2008-2019 (In percent of GDP, unless otherwise indicated)

\begin{tabular}{|c|c|c|c|c|c|c|c|c|c|c|c|c|}
\hline & \multirow{2}{*}{$\begin{array}{c}\text { Actual } \\
2008 \\
\end{array}$} & \multicolumn{10}{|c|}{ Projections } & \multirow[b]{2}{*}{2019} \\
\hline & & 2009 & 2010 & 2011 & 2012 & 2013 & 2014 & 2015 & 2016 & 2017 & 2018 & \\
\hline Public sector debt $1 /$ & 135.6 & 193.0 & 168.2 & 186.0 & 201.1 & 216.4 & 237.5 & 259.7 & 287.8 & 324.5 & 368.2 & 418.1 \\
\hline $\mathrm{o} / \mathrm{w}$ foreign-currency denominated & 83.3 & 153.1 & 130.9 & 154.7 & 177.6 & 200.5 & 221.9 & 244.1 & 272.3 & 309.1 & 353.3 & 403.6 \\
\hline Change in public sector debt & 5.8 & 57.4 & -24.8 & 17.8 & 15.1 & 15.3 & 21.1 & 22.2 & 28.1 & 36.7 & 43.8 & 49.8 \\
\hline Identified debt-creating flows $(4+7+12)$ & -5.5 & 3.2 & -29.7 & 11.1 & 10.4 & 16.6 & 20.3 & 23.3 & 30.6 & 37.4 & 46.9 & 52.3 \\
\hline Primary deficit (negative means surplus) & -3.9 & -13.4 & -7.0 & -6.0 & -4.7 & -4.7 & -4.2 & -4.1 & -3.4 & -3.3 & -3.2 & -3.2 \\
\hline Revenue and grants & 36.4 & 38.3 & 33.6 & 33.7 & 33.4 & 33.4 & 33.2 & 33.2 & 32.7 & 32.4 & 32.0 & 31.5 \\
\hline Primary (noninterest) expenditure & 32.5 & 24.9 & 26.7 & 27.7 & 28.8 & 28.6 & 29.0 & 29.1 & 29.4 & 29.2 & 28.8 & 28.2 \\
\hline Automatic debt dynamics $2 /$ & 1.3 & 20.4 & -21.0 & 18.1 & 16.0 & 22.2 & 24.5 & 27.4 & 33.9 & 40.6 & 50.1 & 55.6 \\
\hline Contribution from interest rate/growth differential 3/ & -20.6 & -12.3 & 5.5 & 11.5 & 12.4 & 15.9 & 20.0 & 23.7 & 29.8 & 36.0 & 44.8 & 52.9 \\
\hline Of which contribution from real GDP growth & 0.9 & 8.4 & -7.2 & -7.8 & -8.6 & -9.3 & -10.0 & -11.0 & -10.9 & -12.0 & -12.1 & -13.8 \\
\hline Contribution from exchange rate depreciation $4 /$ & 21.9 & 32.7 & -26.5 & 6.6 & 3.6 & 6.3 & 4.4 & 3.7 & 4.1 & 4.6 & 5.3 & 2.7 \\
\hline Denominator $=1+g+p+g p$ & 1.3 & 1.2 & 1.1 & 1.1 & 1.1 & 1.1 & 1.1 & 1.1 & 1.1 & 1.1 & 1.1 & 1.1 \\
\hline Other identified debt-creating flows & -2.9 & -3.8 & -1.8 & -0.9 & -0.9 & -0.9 & 0.0 & 0.0 & 0.0 & 0.0 & 0.0 & 0.0 \\
\hline Privatization receipts (negative) & -2.9 & -3.8 & -1.8 & -0.9 & -0.9 & -0.9 & 0.0 & 0.0 & 0.0 & 0.0 & 0.0 & 0.0 \\
\hline Recognition of implicit or contingent liabilities & 0.0 & 0.0 & 0.0 & 0.0 & 0.0 & 0.0 & 0.0 & 0.0 & 0.0 & 0.0 & 0.0 & 0.0 \\
\hline Other (specify, e.g. bank recapitalization) & 0.0 & 0.0 & 0.0 & 0.0 & 0.0 & 0.0 & 0.0 & 0.0 & 0.0 & 0.0 & 0.0 & 0.0 \\
\hline Residual, including asset changes (2-3) & 11.3 & 54.2 & 4.9 & 6.7 & 4.7 & -1.3 & 0.8 & -1.1 & -2.5 & -0.7 & -3.1 & -2.5 \\
\hline Public sector debt-to-revenue ratio $1 /$ & 372.5 & 504.0 & 500.0 & 552.2 & 601.5 & 648.6 & 715.4 & 782.0 & 879.0 & 1000.3 & 1150.6 & 1328.6 \\
\hline Gross financing need $5 /$ & 45.1 & 31.9 & 47.0 & 68.9 & 61.2 & 68.5 & 78.9 & 84.9 & 95.3 & 107.9 & 123.3 & 141.7 \\
\hline \multicolumn{13}{|l|}{ Key Macroeconomic and Fiscal Assumptions } \\
\hline Real GDP growth (in percent) & -0.9 & -7.6 & 4.0 & 5.0 & 5.0 & 5.0 & 5.0 & 5.0 & 4.5 & 4.5 & 4.0 & 4.0 \\
\hline Average nominal interest rate on public debt (in percent) $6 /$ & 7.1 & 10.4 & 11.3 & 14.9 & 15.5 & 16.6 & 18.0 & 18.8 & 19.9 & 21.0 & 21.8 & 22.4 \\
\hline Average nominal interest rate on forex debt (in percent) 6 / & 4.4 & 11.4 & 10.2 & 16.6 & 17.2 & 17.9 & 19.0 & 19.7 & 20.8 & 21.8 & 22.6 & 23.1 \\
\hline Average real interest rate (nominal rate minus change in GDP deflator, in percent) & -21.3 & -21.0 & 7.3 & 12.5 & 12.4 & 13.7 & 15.2 & 15.9 & 17.0 & 18.1 & 18.9 & 19.5 \\
\hline Nominal depreciation of local currency (LC per dollar) & 41.8 & 42.9 & -17.0 & 4.6 & 2.1 & 3.2 & 2.0 & 1.5 & 1.5 & 1.5 & 1.5 & 0.7 \\
\hline Nominal appreciation (increase in US dollar value of local currency, in percent) & -29.5 & -30.0 & 20.5 & -4.4 & -2.1 & -3.1 & -2.0 & -1.5 & -1.5 & -1.5 & -1.5 & -0.6 \\
\hline Inflation rate (GDP deflator, in percent) & 28.4 & 31.4 & 4.0 & 2.4 & 3.1 & 2.9 & 2.9 & 2.9 & 2.9 & 2.9 & 2.9 & 2.9 \\
\hline Growth of real primary spending (deflated by GDP deflator, in percent) & -5.8 & -31.5 & 10.7 & 8.8 & 9.3 & 4.5 & 6.4 & 5.2 & 6.1 & 4.3 & 3.6 & 5.0 \\
\hline Primary deficit & -3.9 & -13.4 & -7.0 & -6.0 & -4.7 & -4.7 & -4.2 & -4.1 & -3.4 & -3.3 & -3.2 & -3.2 \\
\hline \multicolumn{13}{|l|}{ A. Alternative Scenarios } \\
\hline A1. Key variables are at their $h$ & 135.6 & 213.3 & 194.1 & 213.9 & 229.3 & 241.6 & 255.4 & 266.5 & 276.2 & 288.8 & 298.2 & 306.3 \\
\hline A2. No policy change (constant primary balance) in $2008-12$ & 135.6 & 206.7 & 189.2 & 217.0 & 241.2 & 267.7 & 300.7 & 336.3 & 379.9 & 434.8 & 500.1 & 574.6 \\
\hline \multicolumn{13}{|l|}{ B. Bound Tests } \\
\hline B1. Real interest rate is at baseline plus 0 & 135.6 & 195.4 & 174.7 & 197.8 & 219.1 & 242.7 & 273.6 & 307.9 & 351.5 & 408.0 & 477.8 & 563.1 \\
\hline B2. Real GDP growth is at baseline minus two standard deviation & 135.6 & 199.2 & 180.6 & 208.5 & 236.3 & 267.8 & 309.8 & 357.8 & 419.2 & 499.2 & 598.4 & 708.3 \\
\hline B3. Primary balance is at baseline minus one standard deviation & 135.6 & 196.3 & 176.0 & 199.3 & 220.4 & 242.8 & 271.8 & 302.9 & 341.7 & 391.0 & 450.1 & 517.2 \\
\hline B4. Combination of B1-B3 using one-quarter standard deviation shocks & 135.6 & 203.6 & 188.6 & 221.2 & 253.5 & 290.0 & 337.5 & 391.6 & 460.4 & 549.5 & 660.7 & 793.9 \\
\hline B5. One time 15 percent additional real depreciation in 2009 9/ & 135.6 & 220.2 & 191.3 & 210.3 & 226.3 & 243.3 & 266.3 & 290.7 & 322.0 & 362.8 & 412.0 & 471.7 \\
\hline B6. 10 percent of GDP increase in other debt-creating flows in 2009 & 135.6 & 201.3 & 175.5 & 193.8 & 209.5 & 225.8 & 247.9 & 271.3 & 301.0 & 339.6 & 385.8 & 438.3 \\
\hline
\end{tabular}

1/ Public sector covers non-finacial public sector and the IMF loan to the CBS. Debt is on a gross basis. External and domestic financing gaps are assumed to be closed by additional borrowing. $2 /$ Derived as $[(r-p(1+g)-g+a e(1+r)] /(1+g+p+g p))$ times previous period debt ratio, with $r=$ interest rate; $p=$ growth rate of GDP deflator; $g=$ real GDP growth rate; $a=$ share of foreign-currency 2/ Derived as $[(r-p(1+g)-g+a e(1+r)] /(1+g+p+g p))$ times previous period debt ratio, with $r=$ interest rate; $p=$ growth rate of $G D P$
denominated debt; and $\mathrm{e}=$ nominal exchange rate depreciation (measured by increase in local currency value of U.S. dollar).

$3 /$ The real interest rate contribution is derived from the denominator in footnote $2 /$ as $r-\pi(1+g)$ and the real growth contribution as $-g$.

$3 /$ The real interest rate contribution is derived from the denominator in footnote $2 /$ as $r-\pi$
$4 /$ The exchange rate contribution is derived from the numerator in footnote $2 /$ as ae $(1+r)$.

$5 /$ Defined as public sector deficit, plus amortization of medium and long-term public sector debt, plus short-term debt at end of previous period.

5/ Defined as public sector deficit, plus amortization of medium and long-term public

6/ Derived as nominal interest expenditure divided by previous period debt stock.

7/ The key variables include real GDP growth; real interest rate; and primary balance in perc
8/ The implied change in other key variables under this scenario is discussed in the text.

(mext.

(based on GDP deflator).

$10 /$ Assumes that key variables (real GDP growth, real interest rate, and other identified debt-creating flows) remain at the level of the last projection year. 
Figure 1a. Public DSA: Bound Tests for Baseline Scenario (PC Resructuring and Nonconcessional Refinancing) 1/ (Public debt in percent of GDP)
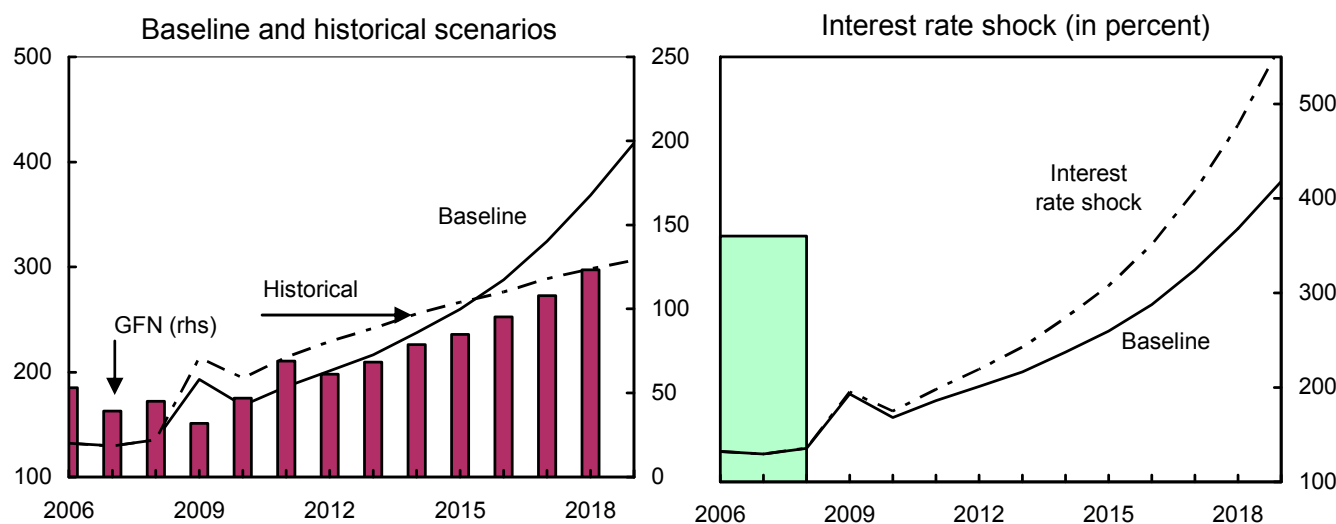

Primary balance shock and no policy change scenarios

Growth shock (in percent per year)

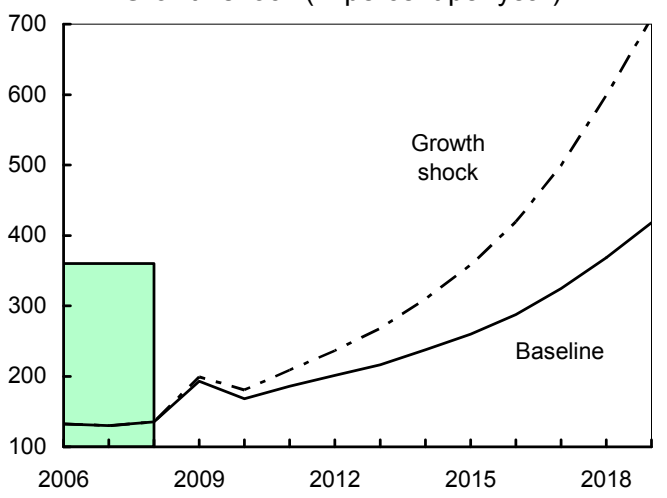

(constant primary balance)
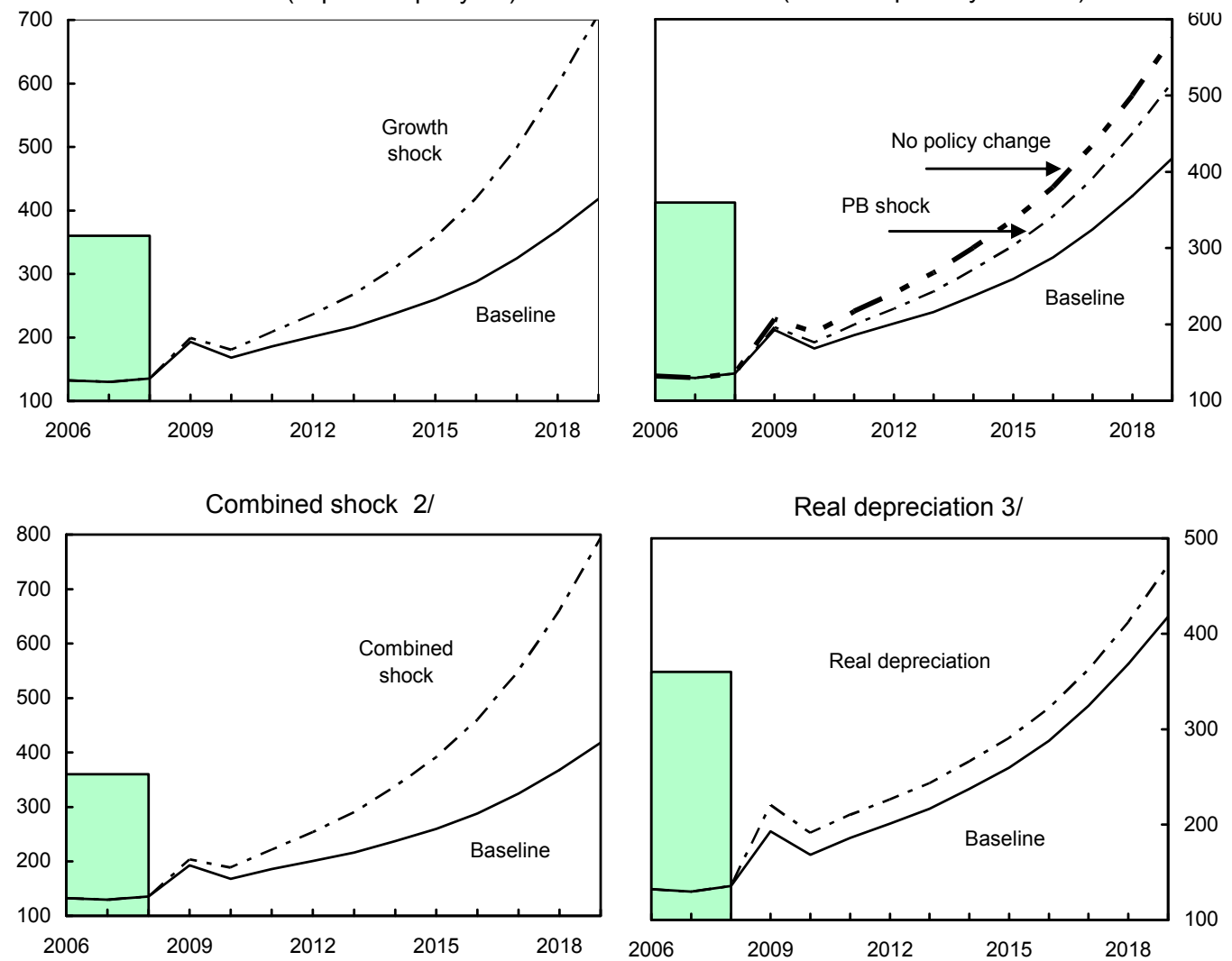

Sources: International Monetary Fund, Country desk data, and staff estimates.

$1 /$ Shaded areas represent actual data. Individual shocks are permanent one standard deviation shocks (two s.d. for growth). Figures in the boxes represent average projections for the respective variables in the baseline and scenario being presented. Ten-year historical average for the variable is also shown.

2/ Permanent 1/4 standard deviation shocks applied to real interest rate, growth rate, and primary balance. 3 / One-time additional real depreciation of 15 percent in 2009, with real depreciation defined as nominal depreciation (measured by percentage fall in dollar value of local currency) minus domestic inflation (based on GDP deflator). 
Table 4. Seychelles: Balance of Payments Alternative Scenario (Assuming Comparabily of Treatment for all Creditors), 2008-19 (Millions of U.S. dollars)

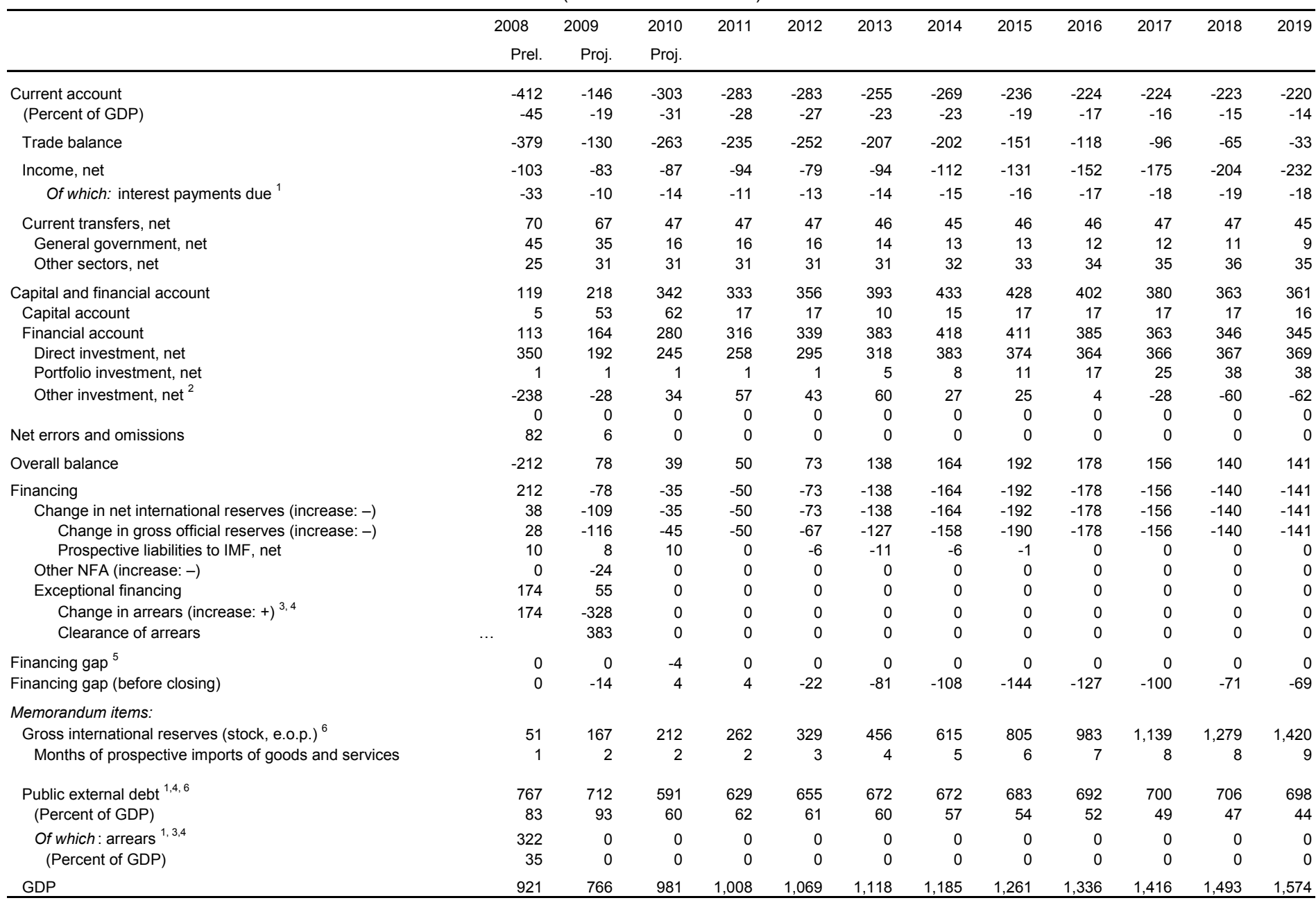

Sources: Central Bank of Seychelles; Ministry of Finance; and IMF staff estimates and projections.

${ }_{1}^{1}$ Projections are based on identified new borrowing and rescheduled Paris Club debt. It assumes restructuring of debt to other creditors on comparable cash flow treatment. Arrears restructuring leads to higher rescheduled interest payments.

${ }^{2}$ Based on Paris Club agreement, the payments of principal and most interest during 2008 and through June 2009 are deferred.

${ }^{3}$ In 2008, includes accelerated promissory notes.

${ }^{4}$ Debt forgivenesss reflects: (i) the first stage of debt reduction (about 22.5) percent in July 2009; (ii) the second stage reduction (about 22.5 percent) in July 2010 on Paris Club debt and assumes debt reduction comparable to the Paris Club agreement to other creditors (including commercial debt). Debt to multilaterals and one commercial creditor (Pride) is excluded from the debt restructuring scenarios.

${ }^{5}$ Negative financing gaps (surpluses) are added to official reserves.

${ }^{6}$ Starting with 2007, refers to CBS gross international reserves net of blocked deposits and project accounts. 
Table 4a. Seychelles: External Debt Sustainability Framework Alternative Scenario (Assuming Comparability of Treatment for all Creditors), 2008-2019 (In percent of GDP, unless otherwise indicated)

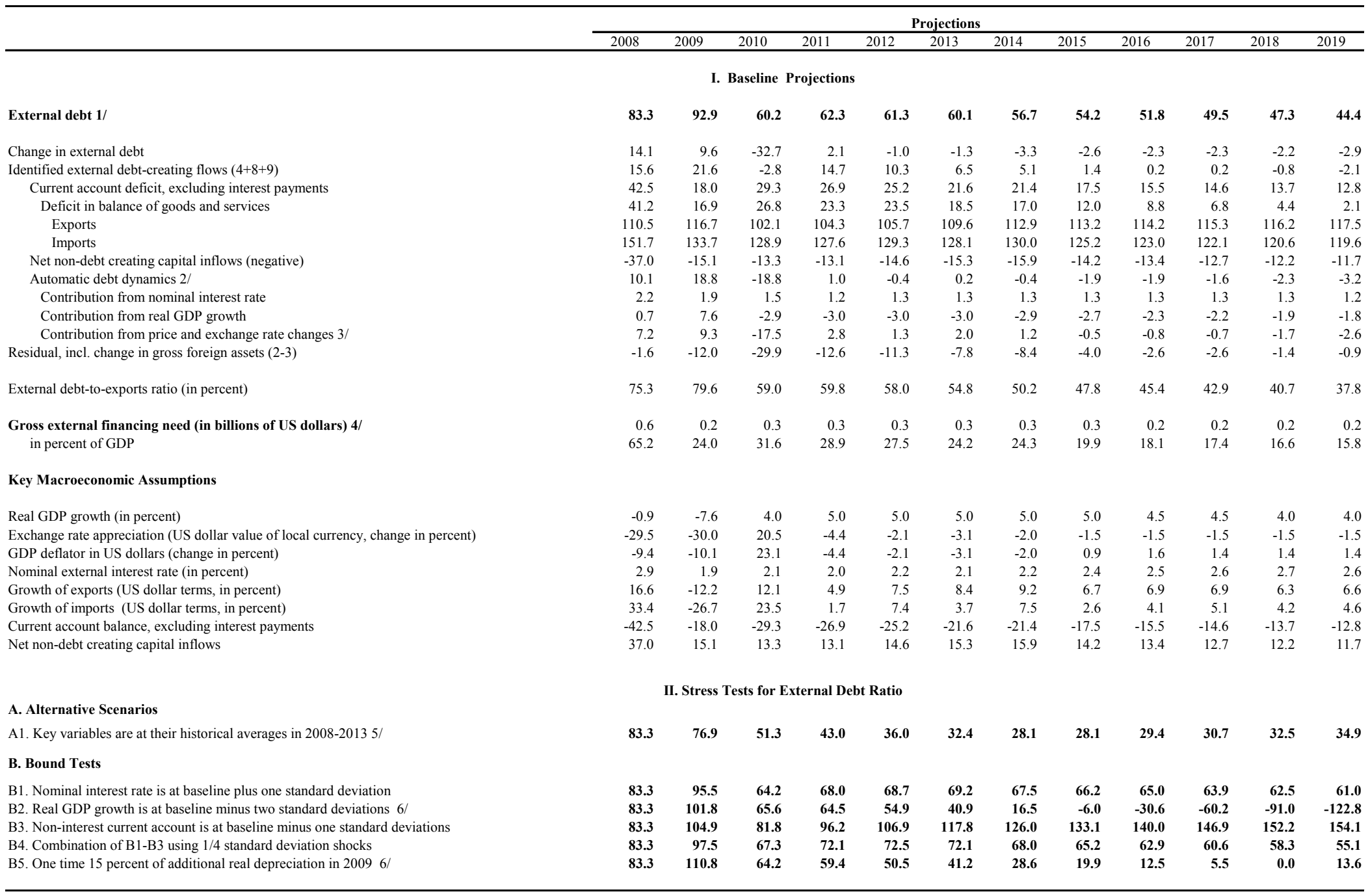

$1 /$ Includes CBS external debt

$2 /$ Derived as $[\mathrm{r}-\mathrm{g}-\rho(1+\mathrm{g})+\varepsilon \alpha(1+\mathrm{r})] /(1+\mathrm{g}+\rho+\mathrm{g} \rho)$ times previous period debt stock, with $\mathrm{r}=$ nominal effective interest rate on external debt; $\rho=$ change in domestic GDP deflator in US dollar terms,

$\mathrm{g}=$ real GDP growth rate, $\mathrm{e}=$ nominal appreciation (increase in dollar value of domestic currency), and $\mathrm{a}=$ share of domestic-currency denominated debt in total external debt.

$3 /$ The contribution from price and exchange rate changes is defined as $[-\rho(1+\mathrm{g})+\varepsilon \alpha(1+\mathrm{r})](1+\mathrm{g}+\rho+\mathrm{g} \rho)$ times previous period debt stock. $\rho$ increases with an appreciating domestic currency $(\varepsilon>0)$

and rising inflation (based on GDP deflator).

4/ Defined as current accourt deficit, plus amortization on medium- and long-term debt, plus short-term debt at end of previous period.

5/ The key variables include real GDP growth; nominal interest rate; dollar deflator growth; and both non-interest current account and non-debt inflows in percent of GDP.

6/ Negative numbers driven by assumptions of partial adjustment, in particular that FDI remains at baseline values (in levels). 
Figure 2. External DSA: Bound Tests for Alternative Scenario (Assuming Comparibility of Treatment for other Creditors), 2006-19

(External debt in percent of GDP)

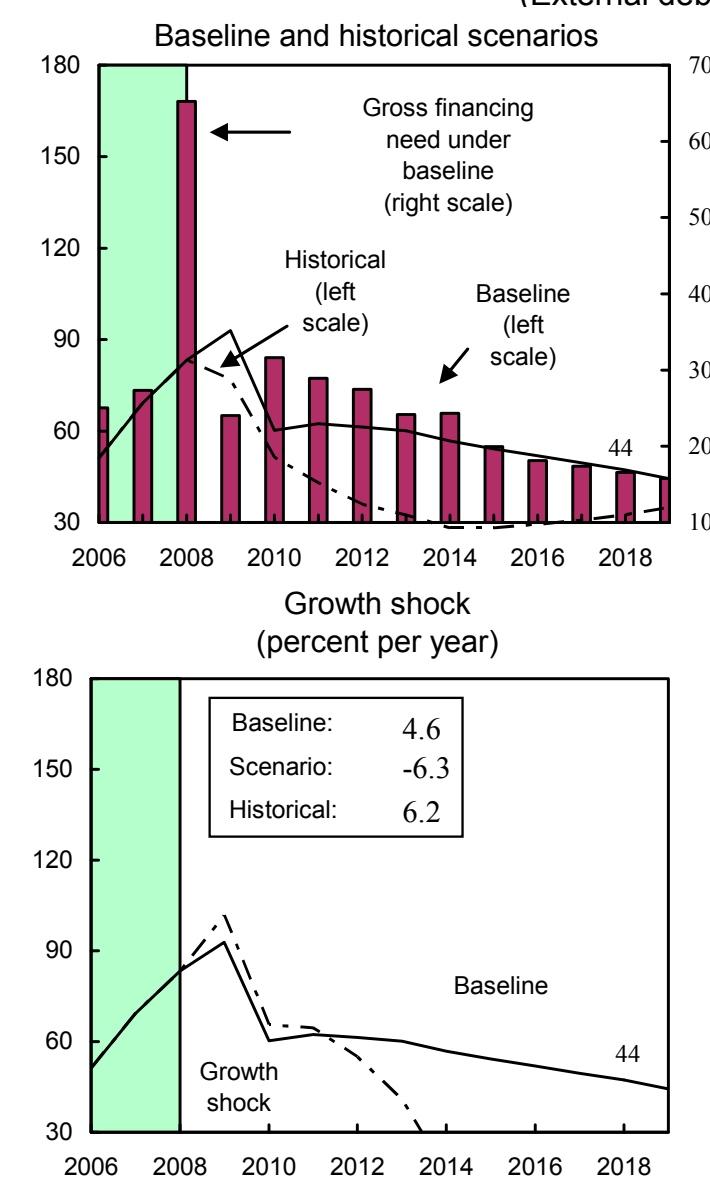

Interest rate shock (percent)
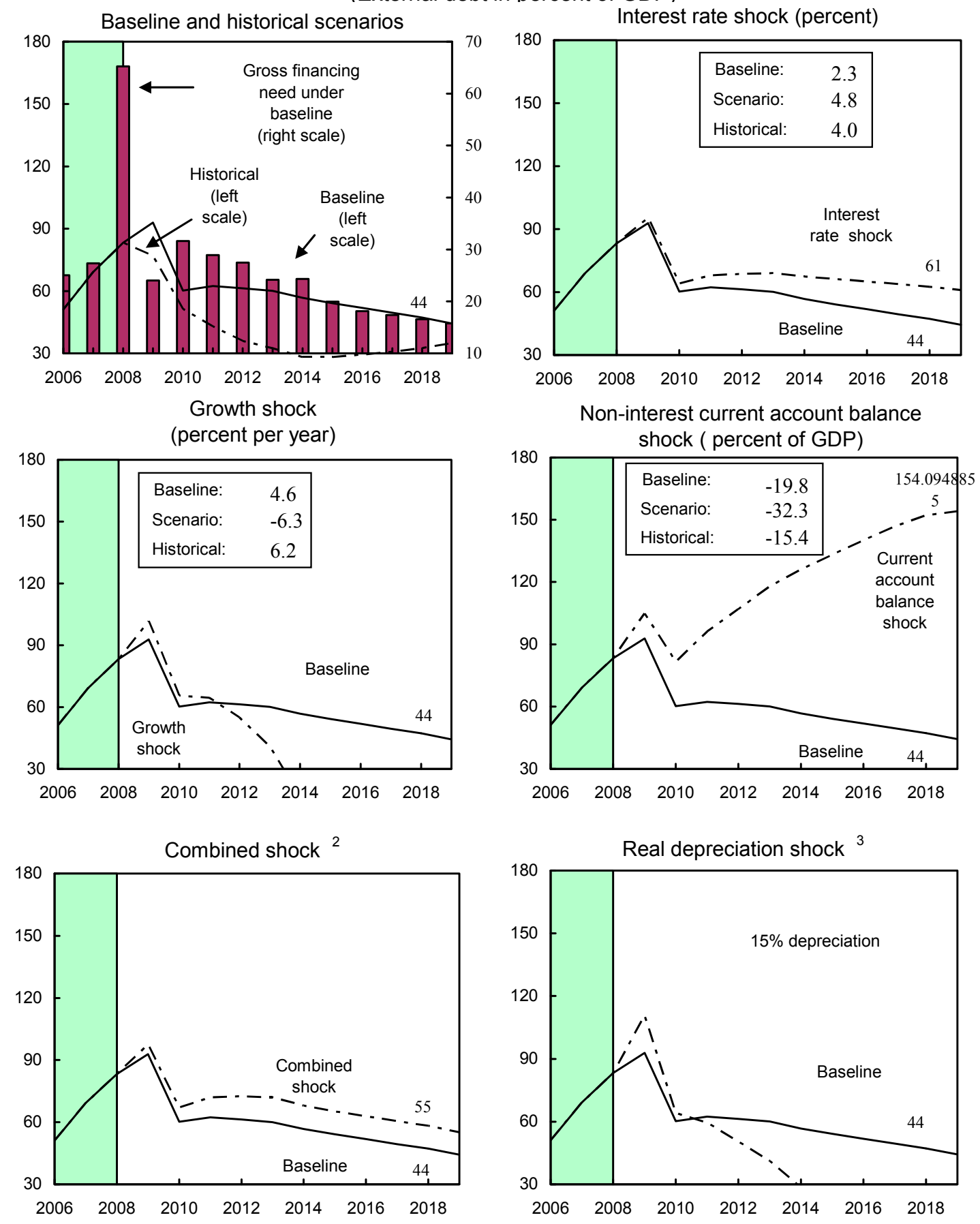

Sources: International Monetary Fund, Country desk data, and staff estimates.

${ }^{1}$ External financing gap is assumed to be closed by external borrowing at LIBOR plus $1300 \mathrm{bps}$, with 3 year maturity. Shaded areas represent actual data. Individual shocks are permanent one standard deviation shocks (two standard deviations for growth shock). Figures in the boxes represent average projections for the respective variables in the baseline and scenario being presented. Ten-year historical average for the variable is also shown.

${ }^{2}$ Permanent $1 / 4$ standard deviation shocks applied to real interest rate, growth rate, and current account balance.

${ }^{3}$ One-time real depreciation of additional 15 percent occurs in 2009. 
Table 4b. Seychelles: Public Sector Debt Sustainability Framework Alternative Scenario (Assuming Comparability of Treatment for all Creditors), $2008-2019$ (In percent of GDP, unless otherwise indicated)

\begin{tabular}{|c|c|c|c|c|c|c|c|c|c|c|c|c|}
\hline & \multirow{2}{*}{$\begin{array}{c}\text { Actual } \\
2008\end{array}$} & \multicolumn{10}{|c|}{ Projections } & \multirow[b]{2}{*}{2019} \\
\hline & & 2009 & 2010 & 2011 & 2012 & 2013 & 2014 & 2015 & 2016 & 2017 & 2018 & \\
\hline $\begin{array}{l}\text { Public sector debt } 1 / \\
\text { o/w foreign-currency denominated }\end{array}$ & $\begin{aligned} 135.6 \\
83.3\end{aligned}$ & $\begin{array}{r}134.2 \\
92.9\end{array}$ & $\begin{array}{ll}97.6 \\
60.2\end{array}$ & $\begin{array}{ll}93.6 \\
62.3\end{array}$ & 84.9 & 76.0 & 72.3 & 69.8 & 67.4 & 65.0 & 62.5 & 59.3 \\
\hline Change in public sector deht & 58 & -15 & -36.6 & -40 & -8.0 & -8.1 & -36 & -26 & -24 & 4.5 & 4.5 & 44.4 \\
\hline Identified debt-creating flows $(4+7+12)$ & -4.5 & -0.7 & -24.7 & -5.4 & $\begin{array}{l}-0.0 \\
-6.9\end{array}$ & -6.3 & -5.2 & -5.0 & $\begin{array}{r}-2.4 \\
-3.9\end{array}$ & $\begin{array}{l}-3.4 \\
-3.6\end{array}$ & -3.3 & $\begin{array}{r}-0.0 \\
-3.4\end{array}$ \\
\hline Primary deficit (negative means surplus) & -2.9 & -11.9 & -7.0 & -6.0 & -4.7 & -4.7 & -4.2 & -4.1 & -3.4 & -3.3 & -3.3 & -3.3 \\
\hline Revenue and grants & 35.5 & 37.9 & 33.7 & 33.7 & 33.5 & 33.4 & 33.2 & 33.2 & 32.9 & 32.8 & 32.6 & 32.4 \\
\hline Primary (noninterest) expenditure & 32.6 & 25.9 & 26.7 & 27.7 & 28.8 & 28.7 & 29.0 & 29.1 & 29.5 & 29.5 & 29.3 & 29.0 \\
\hline Automatic debt dynamics $2 /$ & $\begin{array}{r}3.0 \\
1.3\end{array}$ & 15.0 & $\begin{array}{r}20.1 \\
-15.9\end{array}$ & $\begin{array}{r}2.1 \\
1.6\end{array}$ & $\begin{array}{l}2.0 \\
-1.4\end{array}$ & $\begin{array}{l}2.1 \\
-0.7\end{array}$ & -1.0 & $\begin{array}{l}29.1 \\
-0.9\end{array}$ & $\begin{array}{l}2.5 \\
-0.5\end{array}$ & $\begin{array}{l}2.5 \\
-0.3\end{array}$ & $\begin{array}{r}2.3 \\
0.0\end{array}$ & -0.1 \\
\hline Contribution from interest rate/growth differential $3 /$ & -20.6 & -16.1 & -0.7 & -1.2 & -2.7 & -2.6 & -2.2 & -1.7 & -1.3 & -1.1 & -0.7 & -0.4 \\
\hline Of which contribution from real interest rate & -21.5 & -24.5 & 4.2 & 3.4 & 1.7 & 1.3 & 1.3 & 1.6 & 1.6 & 1.7 & 1.7 & 1.9 \\
\hline Of which contribution from real GDP growth & 0.9 & 8.4 & -5.0 & -4.5 & -4.3 & -3.9 & -3.5 & $\begin{array}{l}1.0 \\
-3.3\end{array}$ & -2.9 & $\begin{array}{l}1.1 \\
-2.8\end{array}$ & -2.4 & $\begin{array}{l}1.3 \\
-2.3\end{array}$ \\
\hline Contribution from exchange rate depreciation $4 /$ & 21.9 & 31.1 & -15.2 & 2.7 & 1.3 & 1.9 & 1.2 & 0.8 & 0.8 & 0.8 & 0.7 & 0.3 \\
\hline Denominator $=1+g+p+g p$ & 1.3 & 1.2 & 1.1 & 1.1 & 1.1 & 1.1 & 1.1 & 1.1 & 1.1 & 1.1 & 1.1 & 1.1 \\
\hline Other identified debt-creating flows & -2.9 & -3.8 & -1.8 & -0.9 & -0.9 & -0.9 & 0.0 & 0.0 & 0.0 & 0.0 & 0.0 & 0.0 \\
\hline Privatization receipts (negative) & -2.9 & -3.8 & -1.8 & -0.9 & -0.9 & -0.9 & 0.0 & 0.0 & 0.0 & 0.0 & 0.0 & 0.0 \\
\hline Recognition of implicit or contingent I & 0.0 & 0.0 & 0.0 & 0.0 & 0.0 & 0.0 & 0.0 & 0.0 & 0.0 & 0.0 & 0.0 & 0.0 \\
\hline Other (specify, e.g. bank recapitaliza & 0.0 & 0.0 & 0.0 & 0.0 & 0.0 & 0.0 & 0.0 & 0.0 & 0.0 & 0.0 & 0.0 & 0.0 \\
\hline Residual, including asset changes (2-3) & 10.3 & -0.8 & -11.9 & 1.4 & -1.8 & $\begin{array}{l}0.0 \\
-2.6\end{array}$ & 1.6 & 2.5 & 1.5 & 1.2 & 0.8 & 0.2 \\
\hline Public sector debt-to-revenue ratio $1 /$ & 382.2 & 354.4 & 289.8 & 277.8 & 253.6 & 227.5 & 217.8 & 210.0 & 204.7 & 198.4 & 191.7 & 181.6 \\
\hline $\begin{array}{l}\text { Gross financing need } 5 / \\
\text { in billions of U.S. dollars }\end{array}$ & $\begin{array}{r}39.5 \\
0.4\end{array}$ & $\begin{array}{r}22.8 \\
0.2\end{array}$ & $\begin{array}{r}32.4 \\
0.3\end{array}$ & $\begin{array}{r}26.4 \\
0.3\end{array}$ & $\begin{array}{r}23.4 \\
0.2\end{array}$ & $\begin{array}{r}19.9 \\
0.2\end{array}$ & $\begin{array}{r}16.4 \\
0.2\end{array}$ & $\begin{array}{r}15.2 \\
0.2\end{array}$ & $\begin{array}{r}14.9 \\
0.2\end{array}$ & $\begin{array}{r}14.1 \\
0.2\end{array}$ & $\begin{array}{r}13.7 \\
0.2\end{array}$ & $\begin{array}{r}14.7 \\
0.2\end{array}$ \\
\hline \multicolumn{13}{|l|}{ Key Macroeconomic and Fiscal Assumptions } \\
\hline Real GDP growth (in percent) & -0.9 & -7.6 & 4.0 & 5.0 & 5.0 & 5.0 & 5.0 & 5.0 & 4.5 & 4.5 & 4.0 & 4.0 \\
\hline Average nominal interest rate on public debt (in percent) $6 /$ & 7.1 & 7.1 & 7.6 & 6.3 & 5.2 & 4.7 & 5.0 & 5.5 & 5.5 & 5.8 & 5.8 & 6.3 \\
\hline Average nominal interest rate on forex debt (in percent) $6 /$ & 4.4 & 5.8 & $\begin{array}{l}1.0 \\
4.1\end{array}$ & 4.6 & 4.2 & 3.9 & 4.5 & 5.1 & 5.1 & 5.4 & 5.5 & 4.2 \\
\hline Average real interest rate (nominal rate minus change in GDP deflator, in percent) & -21.3 & -24.4 & 3.6 & 3.8 & 2.1 & 1.8 & 2.1 & 2.6 & 2.6 & 2.9 & 2.9 & 3.4 \\
\hline Exchange rate (LC per US dollar) & 9.5 & 13.6 & 11.3 & 11.8 & 12.0 & 12.4 & 12.7 & 12.9 & 13.1 & 13.3 & 13.5 & 13.6 \\
\hline Nominal depreciation of local currency (LC per dollar) & $\begin{array}{r}91.0 \\
41.8\end{array}$ & 42.9 & -17.0 & 4.6 & 2.1 & 3.2 & 2.0 & 1.5 & 1.5 & 1.5 & $\begin{array}{r}1.5 \\
\end{array}$ & 0.7 \\
\hline Nominal appreciation (increase in US dollar value of local currency, in percent) & $\begin{array}{l}4.8 \\
-29.5\end{array}$ & $\begin{array}{r}42.9 \\
-30.0\end{array}$ & $\begin{array}{r}-11.0 \\
20.5\end{array}$ & -4.4 & $\begin{array}{l}2.1 \\
-2.1\end{array}$ & $\begin{aligned} 3.2 \\
-3.1\end{aligned}$ & -2.0 & $\begin{array}{r}1.5 \\
-1.5\end{array}$ & -1.5 & -1.5 & -1.5 & -0.6 \\
\hline Inflation rate (GDP deflator, in percent) & 28.4 & 31.4 & 4.0 & 2.4 & 3.1 & 2.9 & 2.9 & 2.9 & 2.9 & 2.9 & 2.9 & 2.9 \\
\hline Growth of real primary spending (deflated by GDP deflator, in percent) & -24.8 & -9.7 & 5.2 & 8.8 & 9.3 & 4.5 & 6.4 & 5.2 & 6.1 & 4.3 & 3.6 & 5.0 \\
\hline Primary deficit & -2.9 & -11.9 & -7.0 & -6.0 & -4.7 & -4.7 & -4.2 & -4.1 & -3.4 & -3.3 & -3.3 & -3.3 \\
\hline \multicolumn{13}{|l|}{ A. Alternative Scenarios } \\
\hline A1. Key variables are at their historical averages in 2008-12 $7 /$ & 135.6 & 163.7 & 137.3 & 146.7 & 151.1 & 156.2 & 166.1 & 177.0 & 187.3 & 197.7 & 208.1 & 216.6 \\
\hline A2. No policy change (constant primary balance) in 2008-12 & 135.6 & 148.2 & 119.4 & 123.7 & 121.3 & 118.9 & 120.8 & 123.6 & 126.1 & 128.6 & 131.4 & 133.3 \\
\hline \multicolumn{13}{|l|}{ B. Bound Tests } \\
\hline B1. Real interest rate is at base & 135.6 & 138.6 & 105.0 & 103.5 & 97.0 & 90.2 & 88.2 & 87.1 & 86.2 & 85.4 & 84.5 & 82.9 \\
\hline B2. Real GDP growth is at $\mathrm{k}$ & 135.6 & 140.2 & 107.4 & 109.1 & 106.2 & 104.1 & 108.8 & 116.1 & 125.1 & 135.6 & 148.0 & 160.7 \\
\hline B3. Primary balance is at baseline & 135.6 & 139.2 & 107.5 & 108.6 & 104.7 & 100.7 & 101.6 & 103.5 & 105.8 & 108.1 & 110.5 & 112.1 \\
\hline B4. Combination of $\mathrm{B} 1-\mathrm{B} 3$ using one-quarter standard deviation shocks & 135.6 & 138.6 & 105.7 & 105.3 & 99.8 & 94.1 & 93.4 & 93.7 & 94.3 & 94.9 & 95.6 & 95.5 \\
\hline B5. One time 15 percent additional real depreciation in $20099 /$ & 135.6 & 162.0 & 121.6 & 116.9 & 106.8 & 96.7 & 91.6 & 87.6 & 83.8 & 80.2 & 76.6 & 72.1 \\
\hline B6. 10 percent of GDP increase in other debt-creating flows in 2009 & 135.6 & 144.3 & 107.0 & 103.2 & 94.4 & 85.6 & 81.7 & 79.0 & 76.5 & 74.0 & 71.5 & 68.2 \\
\hline
\end{tabular}

1/ Public sector covers non-finacial public sector and the IMF loan to the CBS. Debt is on a gross basis. External and domestic financing gaps are assumed to be closed by additional borrowing.

$2 /$ Derived as $[(r-p(1+g)-g+a(1+r) /(1+g+p+g p))$ times previous period debt ratio, with $r=$ interest rate; $p=$ growth rate of GDP deflator; $g=$ real GDP growth rate; $a=$ share of foreign-currency denominated debt; and $\mathrm{e}=$ nominal exchange rate depreciation (measured by increase in local currency value of U.S. dollar).

$3 /$ The real interest rate contribution is derived from the denominator in footnote $2 /$ as $r-\pi(1+g)$ and the real growth contribution as $-g$.

4/ The exchange rate contribution is derived from the numerator in footnote $2 /$ as ae(1+r).

5/ Defined as public sector deficit, plus amortization of medium and long-term public sector debt, plus short-term debt at end of previous period.

/ Derived as nominal interest expenditure divided by previous period debt stock.

7/ The key variables include real

(text

(based on GDP deflator).

$10 /$ Assumes that key variables (real GDP growth, real interest rate, and other identified debt-creating flows) remain at the level of the last projection year. 
Figure 2a. Public DSA: Bound Tests for Alternative Scenario (Assuming Comparability of Treatment for other Creditors)1/ (Public debt in percent of GDP)
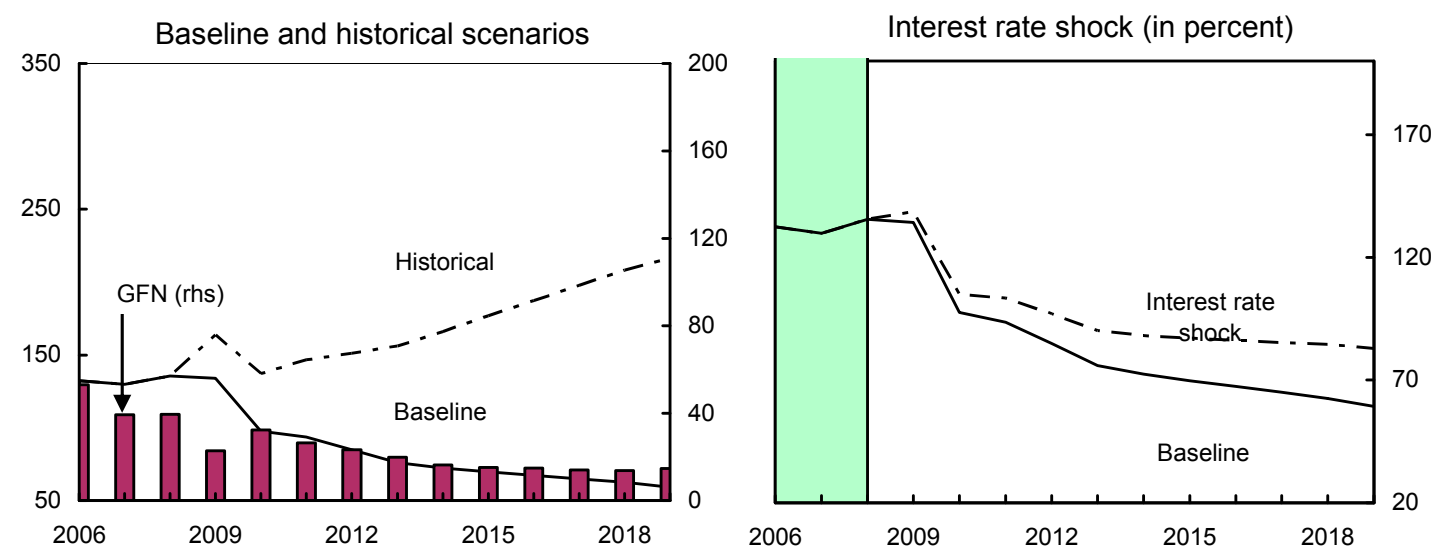

Growth shock (in percent per year)

Primary balance shock and no policy change scenarios
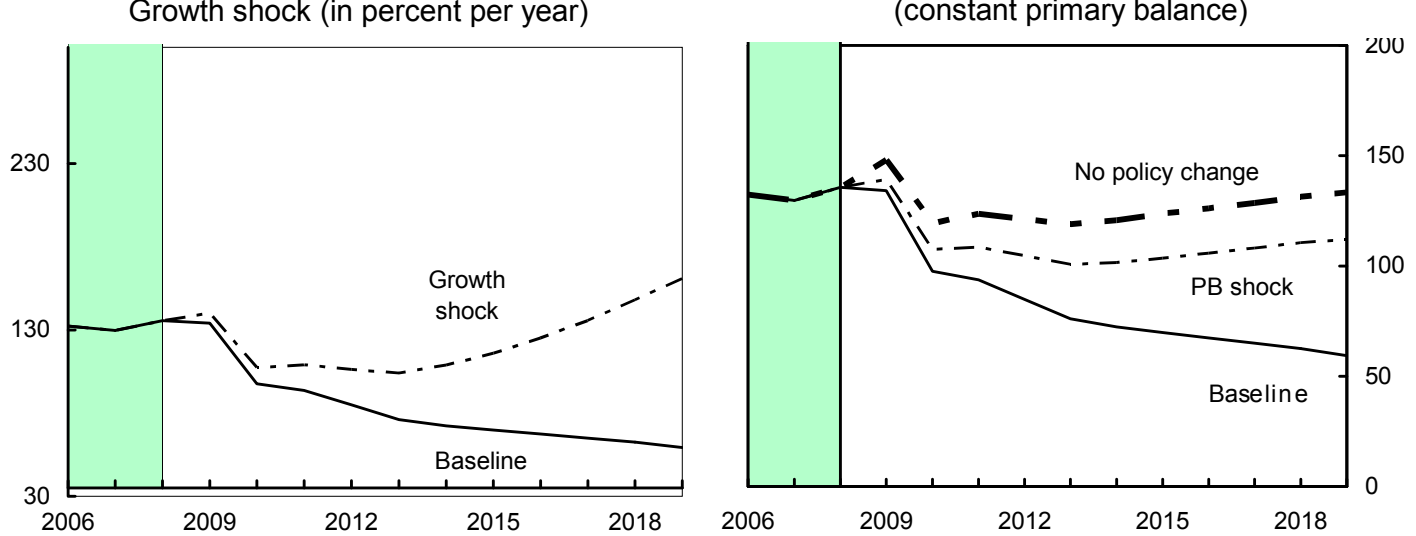

Combined shock 2/
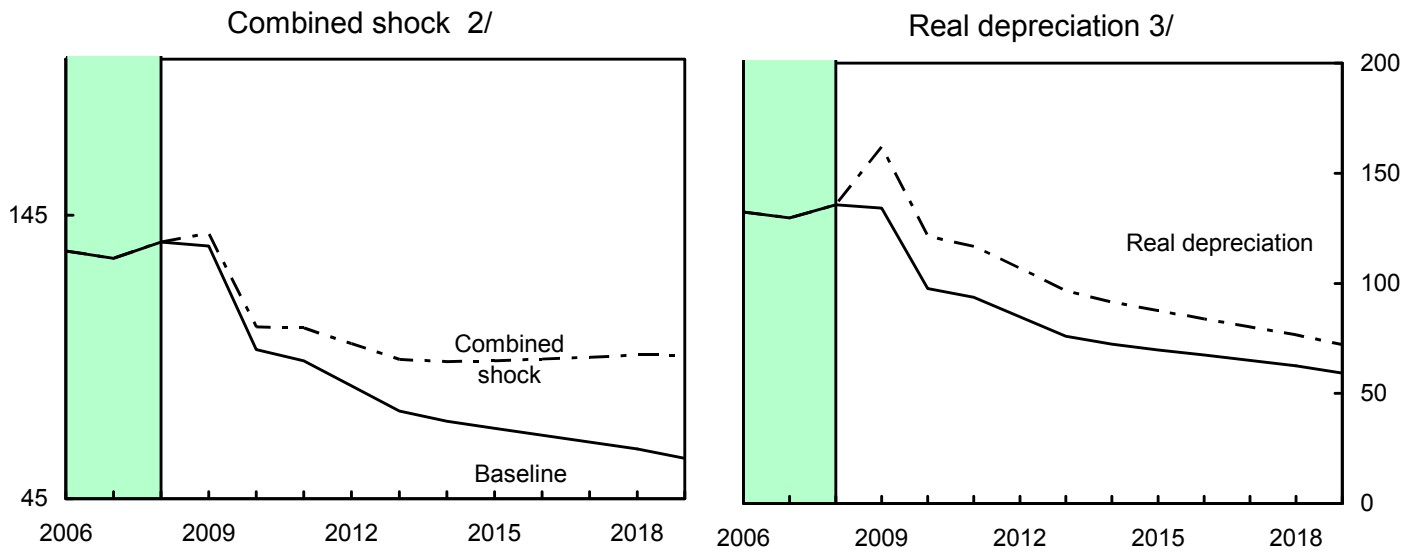

Sources: International Monetary Fund, Country desk data, and staff estimates.

$1 /$ Shaded areas represent actual data. Individual shocks are permanent one standard deviation shocks (two s.d. for growth). Figures in the boxes represent average projections for the respective variables in the baseline and scenario being presented. Ten-year historical average for the variable is also shown.

2/ Permanent 1/4 standard deviation shocks applied to real interest rate, growth rate, and primary balance. $3 /$ One-time additional real depreciation of 15 percent in 2009 , with real depreciation defined as nominal depreciation (measured by percentage fall in dollar value of local currency) minus domestic inflation (based on GDP deflator). 
INTERNATIONAL MONETARY FUND

\section{SEYCHELLES}

Third Review Under the Stand-By Arrangement, Financing Assurances Review, Cancellation of the Stand-By-Arrangement, and Request for an Extended Arrangement -Informational Annex

Prepared by the African Department

(In consultation with other departments)

December 8, 2009

Contents

Page

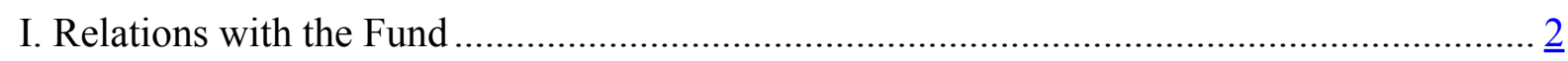

II. Joint World Bank-IMF Work Program, 2009-10 …………………………………........ $\underline{6}$

III. Relations with the African Development Bank Group ................................................... $\underline{8}$ 


\section{SEYCHELLES: RELATIONS WITH THE FUND}

(As of October 31, 2009)

I. Membership Status: Joined 6/30/77. Article VIII.

II. General Resources Account :

Quota

Fund holdings of currency

Reserve Position in Fund

III. SDR Department:

Net cumulative allocation

Holdings

IV. Outstanding Purchases and

Loans:

\begin{tabular}{lll} 
SDR Million & & \% Quota \\
\hline 8.80 & & 100.0 \\
16.72 & & 189.97 \\
0.00 & 0.04
\end{tabular}

$\underline{\text { SDR Million }}$

8.28

\% Allocations

100.0

7.90

95.37

7.92

90.00

$\underline{\text { SDR Million }}$

V. Financial Arrangements:

Type Arrangement Expiration Amount Approved Amount Drawn

$\begin{array}{llll}\text { Stand-By } & \text { Nov.14, } 2008 & \text { Nov. 13, 2010 } & 17.60\end{array}$

VI. Projected Obligations to the Fund (SDR Million: based on existing use of resources and present holdings of SDRs):

\begin{tabular}{lcccc}
\multicolumn{5}{c}{ Forthcoming } \\
\hline $\mathbf{2 0 1 0}$ & $\mathbf{2 0 1 1}$ & $\mathbf{2 0 1 2}$ & $\mathbf{2 0 1 3}$ & $\mathbf{2 0 1 4}$ \\
& & 3.41 & 4.40 & 0.99 \\
0.11 & 0.11 & 0.10 & 0.05 & 0.01 \\
0.11 & 0.11 & 3.51 & 4.45 & 0.10 \\
\hline
\end{tabular}


VII. Implementation of HIPC Initiative: Not applicable

\section{Safeguards Assessments:}

As part of the Stand-By Arrangement, a safeguards assessment mission was conducted in September 2008. It found high risks in all areas of the central bank (CBS) safeguards assessment framework, including significant weaknesses in financial reporting that were not identified by the external auditor and internal control vulnerabilities. The authorities are implementing remedial measures including steps monitored under the program. In late November the CBS appointed a new external auditor, PricewaterhouseCoopers, on a five-year contract. The authorities continue to make strong progress in addressing the areas of concern identified in the safeguards assessment, including a new central bank act that meets international best practice.

IX. Exchange Rate Arrangement:

The authorities launched a comprehensive reform effort in November 2008, with reforms including a fundamental liberalization of the exchange regime. The exchange market liberalization resulted in all the elimination of restrictions on the making of payments and transfers for current international transactions that are subject to Fund approval under Article VIII Sections 2, 3, and 4. The exchange rate policy is a float. On December 1, 2009, US\$1 = SR 11.08 (mid rate).

X. Article IV Consultations:

Seychelles is currently under a two year Stand-By Arrangement, with semi-annual reviews. 
XI. Technical Assistance (2004-March 2009):

\begin{tabular}{|c|c|c|c|}
\hline Department & $\begin{array}{l}\text { Head of } \\
\text { Mission }\end{array}$ & Subject & Date \\
\hline STA & Mr. Freeman & National Accounts/CPI & May/June 2005 \\
\hline MFD/LEG & Mr. Lonnberg & $\begin{array}{l}\text { Strengthening Capacity in } \\
\text { Critical Central Banking } \\
\text { Areas, including } \\
\text { AML/CFT-Legal, } \\
\text { Institutional, and } \\
\text { Supervisory Frameworks }\end{array}$ & July 2006 \\
\hline LEG & Mr. Beekarry & $\begin{array}{l}\text { Advise on AML/CFT } \\
\text { measures and the } \\
\text { establishment of the FIU }\end{array}$ & $\begin{array}{l}\text { September/October } \\
2006\end{array}$ \\
\hline STA & Mr. Alexander & $\begin{array}{l}\text { National } \\
\text { accounts/CPI/GDDS }\end{array}$ & $\begin{array}{l}\text { October/ November } \\
2006\end{array}$ \\
\hline MCM & Mr. Bartholomew & $\begin{array}{l}\text { Multipurpose mission: } \\
\text { Monetary operations, } \\
\text { monetary research, } \\
\text { banking supervision, } \\
\text { payment systems, foreign } \\
\text { exchange markets, and } \\
\text { nonbank financial } \\
\text { institution supervision. }\end{array}$ & $\begin{array}{l}\text { November/ } \\
\text { December } 2007\end{array}$ \\
\hline STA & Mr. Dessart & $\begin{array}{l}\text { Dissemination of GDDS } \\
\text { National Summary Data } \\
\text { Page }\end{array}$ & April 2008 \\
\hline STA & Mr. Armknecht & Consumer price index & April 2008 \\
\hline MCM & Mr. Faulk & $\begin{array}{l}\text { Banking supervision, } \\
\text { drafting of new financial } \\
\text { sector related regulations }\end{array}$ & April 2008 \\
\hline $\mathrm{MCM}$ & Mr. Robotham & $\begin{array}{l}\text { National payment } \\
\text { systems project }\end{array}$ & May/June 2008 \\
\hline FAD & Mr. Khemani & $\begin{array}{l}\text { Strengthening } \\
\text { expenditure } \\
\text { rationalization and budget } \\
\text { management }\end{array}$ & July 2008 \\
\hline
\end{tabular}




\begin{tabular}{|c|c|c|c|}
\hline Department & Head of Mission & Subject & Date \\
\hline LEG & Mr. Baban & $\begin{array}{l}\text { Exchange Rate and } \\
\text { Exchange Control } \\
\text { Regimes }\end{array}$ & September 2008 \\
\hline FIN & Mr. Hauge & Safeguards assessment & October 2008 \\
\hline FAD & Mr. Krelove & Reform of Tax Policy & $\begin{array}{l}\text { January/February } \\
2009\end{array}$ \\
\hline MCM & Mr. Bartholomew & $\begin{array}{l}\text { Central Bank Capacity } \\
\text { Building after the } 2008 \\
\text { Float }\end{array}$ & February 2009 \\
\hline LEG & Mr. Baban & $\begin{array}{l}\text { Reform of Exchange Rate } \\
\text { Law }\end{array}$ & May 2009 \\
\hline STA & Mr. Jones & $\begin{array}{l}\text { Government Finance } \\
\text { Statistics }\end{array}$ & May 2009 \\
\hline MCM & Mr. Faulk & Bank Supervision & June 2009 \\
\hline MCM & Mr. Vollan & Monetary Operations & June 2009 \\
\hline $\mathrm{MCM}$ & Mr. Robotham & Payments Systems & June 2009 \\
\hline FAD & Mr. Ljungman & $\begin{array}{l}\text { Public Financial } \\
\text { Management }\end{array}$ & July 2009 \\
\hline LEG & Mr. Burns & Fiscal Law & July 2009 \\
\hline FAD & David Kloeden & Tax Administration & September 2009 \\
\hline MCM & Mr. Sullivan & Accounting & October 2009 \\
\hline $\mathrm{MCM}$ & Mr. Faulk & Bank Supervision & October 2009 \\
\hline MCM & Mr. Robotham & Payments Systems & November 2009 \\
\hline
\end{tabular}

XII. Resident Representative: None 
II. Seychelles: Joint World BANK-IMF Work Program, 2009-10

(As of November 30, 2009)

\begin{tabular}{|c|c|c|c|}
\hline Title & Products & $\begin{array}{l}\text { Provisional timing of } \\
\text { mission }\end{array}$ & \begin{tabular}{|l|} 
Expected delivery \\
date
\end{tabular} \\
\hline \multicolumn{4}{|c|}{ A. Mutual Information on Relevant Work Programs } \\
\hline Bank & $\begin{array}{l}\text { A. Strategy and analytical work } \\
\text { Public Expenditure Review (PER, Phase 2) } \\
\text { Update to FIAS report (improving the business } \\
\text { environment and private sector development) } \\
\text { Joint Country Procurement Assessment Report } \\
\text { (CPAR) and Country Financial Accountability } \\
\text { Assessment (CFAA) } \\
\text { Tuna industry management (TA - P118247) } \\
\text { National Climate Change Strategy (TA - P118246) } \\
\text { B. Ongoing and new projects } \\
\text { GEF grant implementation (tsunami reconstruction, } \\
\text { disaster management) } \\
\text { Development Policy Loan (DPL 2) } \\
\text { Statistical Capacity Building Project (STATCAP) }\end{array}$ & $\begin{array}{l}\text { July } 2010 \\
\text { n.a. } \\
\text { March } 2010 \\
\text { March } 2010 \\
\text { March } 2010\end{array}$ & $\begin{array}{l}\text { February } 2010 \\
\text { February } 2010 \\
\text { Ongoing } \\
\text { September } 2010 \\
\text { April } 2011\end{array}$ \\
\hline Fund & $\begin{array}{l}\text { Program and surveillance work } \\
\text { 1. Third review under the SBA } \\
\text { 2. Discussions on medium-term macroeconomic and } \\
\text { structural reform program for } 2010-12 \text {, supported by a } \\
\text { successor arrangement under the EFF. } \\
\text { 3. First Review under EFF } \\
\text { 4. Second Review under EFF } \\
\text { Technical assistance and capacity building } \\
\text { 1. FAD technical assistance mission on tax } \\
\text { administration. } \\
\text { 2. FAD technical assistance on tax and customs reform } \\
\text { 3. MCM technical assistance mission on payments } \\
\text { systems. } \\
\text { 4. MCM technical assistance on reforming the } \\
\text { monetary policy framework (long-term monetary } \\
\text { advisor, automation of interbank market, reserve } \\
\text { money management) strengthening bank supervision, } \\
\text { and CBS governance. }\end{array}$ & $\begin{array}{l}\text { October } 2009 \\
\text { October } 2009 \\
\text { May } 2010 \\
\text { October } 2010 \\
\text { September } 2009 \\
\text { Ongoing } \\
\text { November } 2009 \\
\text { Ongoing }\end{array}$ & $\begin{array}{l}\text { December, } 2009 \\
\text { December, } 2009 \\
\text { June } 2010 \\
\text { December } 2010 \\
\text { December } 2009 \\
\text { Ongoing } \\
\text { January } 2010 \\
\text { Ongoing }\end{array}$ \\
\hline
\end{tabular}




\begin{tabular}{|l|l|l|l|}
\hline Title & Products & $\begin{array}{l}\text { Provisional timing of } \\
\text { mission }\end{array}$ & $\begin{array}{l}\text { Expected delivery } \\
\text { date }\end{array}$ \\
\hline \multicolumn{2}{|c|}{ B. Requests for Work Program inputs } \\
\hline $\begin{array}{l}\text { Bank } \\
\text { request to } \\
\text { Fund }\end{array}$ & $\begin{array}{l}\text { 1. Medium-term macro-economic and fiscal framework } \\
\text { to inform Public Expenditure Reviews } \\
\text { 2. Debt Sustainability Analysis }\end{array}$ & n.a. & Ongoing \\
\hline $\begin{array}{l}\text { Fund } \\
\text { request to } \\
\text { Bank }\end{array}$ & $\begin{array}{l}\text { 1. Assessment of the social safety net and enhancing } \\
\text { targeting of social assistance } \\
\text { 2. Policy note on parastatal reform and privatization. } \\
\text { 3. Strategy on improving business environment and } \\
\text { promoting private sector investment } \\
\text { 4. New wage grid proposal for public sector } \\
\text { 5. IFC assistance on privatization plans for Nouvobanq } \\
\text { and Savings Bank } \\
\text { 6. Develop strategy and policy recommendations to } \\
\text { improve transparency and management of the fisheries } \\
\text { sector } \\
\text { 7. FIRST Initiative support for strategic reform of } \\
\text { government owned non-bank financial institutions }\end{array}$ & $\begin{array}{l}\text { October 2009 } \\
\text { October 2009 }\end{array}$ & October 2009 \\
\hline \multicolumn{1}{|c|}{ C. Agreement on Joint Products and Missions } \\
\hline $\begin{array}{l}\text { Joint } \\
\text { products }\end{array}$ & $\begin{array}{l}\text { Joint mission to discuss a medium-term structural } \\
\text { reform agenda focusing on: social safety net targeting; } \\
\text { civil service reform, parastatal reform; and promoting } \\
\text { private sector investment }\end{array}$ & October 2009 & December 2009 \\
\hline
\end{tabular}




\title{
III. SEyChelles: Relations With the African DeVelopMent Bank Group
}

\author{
(As of November 16, 2009)
}

The African Development Bank Group commenced operations with the Republic of Seychelles in 1978 and has since approved 19 operations comprising 15 projects, one study and three lines of credit for the country. Cumulative approvals as of October 2008 (there were no approvals between 2001 and 2006 when the country was under sanctions) amounted to UA 84.87 million, ${ }^{1}$ of which 73 percent was from the African Development Bank (ADB), 14 percent from the Nigeria Trust Fund (NTF), and the remaining 13 percent from the African Development Fund (ADF) (Table 1). Reflecting government priorities, most of the operations were in the social sector (46 percent). This was followed by the financial sector (16 percent), agricultural sector (15 percent), transportation (13 percent), water supply and sanitation (10 percent) and industrial sector (1 percent). Before sanctions were imposed, about 76.8 percent of the total commitments on all approved loans had been disbursed and the balance of 23.2 percent was cancelled after sanctions were applied.

The country was under sanctions with the African Development Bank Group since February 16,2000 due to the arrears situation. The country cleared all the arrears (amounting to US\$46.75 million) in November 2006 and the African Development Bank Group consequently lifted all sanctions and started discussions on possible reengagement with the country. The process was boosted by the launching of a comprehensive reform programme by the Government in November 2008. Following this, an Interim Strategy Note was approved by the Board on April 22, 2009. The ISN covers 2009 and 2010 and focuses on a single pillar namely supporting the Government's reform programme to achieve sustainable growth. The lending programme during this period consists of a policy-based operation in the form of a budget support loan amounting to US\$20 million. The operation is under preparation and it is expected to be disbursed in two equal tranches of US\$10 million in 2009 and 2010. In addition, there is an ongoing non-lending programme under which the Bank Group is providing grant resources to the country, from the African Water Facility and the Middle Income Countries' (MIC) Technical Assistance Fund. Under the African Water Facility, a grant amounting to $€ 955,000$ was signed in May 2008 to finance the preparation of the Water Development Plan aimed at improved water management and governance, and identifying priority investment projects for funding, in order to meet Seychelles' water needs up to 2030. The study is expected to be completed by December 2009. Under the MIC Technical Assistance Fund two grants have been approved to finance: (i) a study (amounting to UA 600,000) approved in December 2008 aimed mainly to support human resource

\footnotetext{
${ }^{1}$ UA $1=$ US\$ 1.54805 as at June 5, 2009.
} 
development, training and capacity building as well as the establishment of the Seychelles University Foundation; and (ii) a study (amounting to US\$ 500,000) approved in February 2009 to undertake the feasibility on a Submarine Cable System to link Seychelles to East Africa.

A policy based partial guarantee operation for US\$10 million was approved on December 12, 2009 in support of an exchange bond offered to external commercial creditors in the context of the public debt restructuring effort.

Table 1. Operations Summary as at 5 June 2009*

Operations since: 1978

Total Number of operations 19: 15 Projects, 1 Study; 3 Lines of Credit

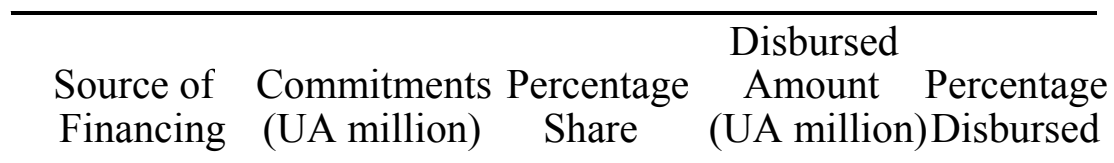

\begin{tabular}{lcccc}
\hline ADB & 62.19 & 73 & 44.12 & 70.9 \\
ADF/TAF & 10.68 & 13 & 10.68 & 100.0 \\
NTF & 12.00 & 14 & 10.39 & 86.6 \\
Total & 84.87 & 100 & 65.19 & 76.8 \\
\hline
\end{tabular}

* Excludes the grants from the African Water Facility and the MIC Technical Assistance Facility due to their unique nature and small magnitude. 


\section{Statement by the IMF Representative December 18, 2009}

This statement provides information that has become available since the staff report was issued. This information does not change the thrust of the staff appraisal.

As anticipated in the staff report, on December 7, 2009 the authorities launched a debt exchange offer aimed at holders of external bonds, notes, and certain commercial bank loans (Box). Discussions with non-Paris Club official bilaterals and other external commercial creditors, largely banks, are also advancing. The authorities have been very conscious of the need to ensure comparability of treatment across creditor groups and are confident that the offer is comparable to terms agreed with Paris Club creditors. Should the offer be successfully concluded, some 70 percent of the end-2008 stock of external public debt will have been restructured.

\section{Box: Commercial Bond Exchange Offer}

The offer covers about US\$320 million face value of remaining commercial external debt, including the US\$230 million Eurobond, the $€ 54.75$ million amortizing note, and two other external commercial debts totaling US\$9 million. ${ }^{1}$

$>$ In exchange for the extinguishing of their claims, creditors are offered the option of a discounted bond (for 50 percent of principal amount tendered) or a par value bond. The offer expires January 14, 2010, with final settlement in mid-February 2010.

$>$ The discount bond has a final maturity of 2026, with a grace period through 2015. Interest is fixed at 3 percent with step-ups in 2012, 2015, and 2018 to 5, 7, and 8 percent, respectively.

$>$ The par bond option has a maturity extended through 2041, with a grace period through 2037. Interest is fixed at 2 percent. The par bonds would only be issued if creditor tenders for this option would result in at least US\$50 million being issued.

$>$ A goodwill cash payment will be made to tendering creditors in April 2010, in lieu of a portion of past due interest on the outstanding debt covered by the offer. The total cash payment will amount to about US\$18 million.

The offer is in line with program expectations and objectives. The program baseline assumes the exchange offer, and related cash payments, would be concluded by end-2009. The program has automatic adjustors to program targets on net international reserves to accommodate the exact timing of the settlement of the offer, expected in February 2010.

\footnotetext{
${ }^{1}$ The debt subject to the offer represents about 78 percent of the value of public external commercial debt outstanding, including arrears.
} 
Staff have updated the debt sustainability analysis on the basis of the authorities' offer and the results are very close to those shown in the restructuring scenario in the staff report Appendix II. Public external and overall debt ratios over the 2010-19 period range between 0.5-3.5 percent of GDP lower that in the staff report Appendix II. The standardized bounds test are materially the same as in the staff report, which indicate that even after full restructuring, Seychelles would remain very vulnerable to a variety of shocks. 
Press Release No. 09/472

International Monetary Fund

FOR IMMEDIATE RELEASE

Washington, D.C. 20431 USA

December 22, 2009

\section{IMF Executive Board Approves US\$31.1 Million Extended Fund Facility Arrangement for Seychelles, Completes Final Review Under Stand-By Arrangement}

The Executive Board of the International Monetary Fund (IMF) has approved a three-year, SDR 19.8 million (about US\$31.1 million) arrangement under the Extended Fund Facility (EFF) for the Seychelles to support the authorities' economic program in 2010-12. The approval makes available a disbursement of SDR 0.88 million (about US\$1.38 million).

The Executive Board also completed the third and final review under the Stand-By Arrangement (SBA). The completion of the review enables a disbursement amounting to SDR 2.2 million (about US\$3.46 million). The SBA was approved on November 14, 2008 (See Press Release No. 08/282). Subsequent to the completion of the third SBA review, and approval of the EFF, the SBA was cancelled.

The authorities' EFF-supported program aims at putting public finances on a sustainable footing and durably raising growth. The key objectives are to support the public debt restructuring process and reestablish external sustainability; accompany the second generation structural reforms aimed at securing macroeconomic stability and raising growth performance; and put in place a macroeconomic framework to guide policies through 2012 .

Following the Executive Board discussion of the Seychelles on December 18, Mr. Takatoshi Kato, Deputy Managing Director and Acting Chair, issued the following statement:

"The Seychelles authorities have made remarkable advances over the last twelve months in addressing long-standing economic problems and establishing macroeconomic stability. Inflation has been near zero and economic recovery is expected to gain traction in 2010 . Program implementation has been exemplary, even in the face of headwinds from the global recession, backed by strong ownership and broad public support. Progress has also been made in negotiations with external creditors aimed at achieving a restructuring compatible with Seychelles' payments capacity.

Washington, D.C. 20431 • Telephone 202-623-7100 • Fax 202-623-6772 • www.imf.org 
“ The strong fiscal adjustment in 2009 played a key role in stabilizing the economy. Good progress has also been made in strengthening public financial management, including through the introduction of a treasury single account. The 2010 budget represents another major step forward in improving transparency and the performance of the public sector and in launching a major tax reform. The new market-based monetary policy has been instrumental in achieving price stability. The flexible exchange rate regime, which has made an important contribution to stabilization and eased the burden on monetary policy, remains appropriate. In view of the very good progress achieved so far, the focus now shifts to implementation of second generation reforms.

"The new three-year EFF arrangement puts in place a macroeconomic framework to guide policies through 2012 and support the authorities' medium-term reform agenda. The key objectives are to consolidate macroeconomic stability, remove constraints to growth, and achieve public debt sustainability. Major improvements in public sector governance practices are needed over the medium term to contain fiscal risks, raise productivity, and reduce the size of the state. It will be important to press ahead with efforts to strengthen financial discipline and accountability in the parastatal sector, bolster the financial system, overhaul tax policy and administration, reinforce their debt management capacity, and pursue public external debt restructuring negotiations," added M. Kato.

\section{Annex}

\section{Recent Economic Developments}

The global recession and piracy in the Indian Ocean hit Seychelles hard in 2009, but there are now early signs of a revival of business activity and employment creation. Real GDP is projected to contract by $7.5 \%$ in 2009 , an improvement on the 10.7 percent contraction projected in June, as the reduction in tourism earnings and related construction activity is attenuating. The economic recovery is expected to strengthen, with economic growth rising to 4 percent in 2010. Inflation has been near zero since March and the exchange rate has appreciated from lows early in the year. Official external reserves continue to recover from near depleted levels.

The government has exceeded its fiscal targets this year with a primary surplus of 13.4 percent of GDP in the first nine months of 2009. Expenditure has been tightly controlled and revenue has held up well despite the difficult economic environment. Social assistance outlays have risen during the year, but have remained within budget allocations.

The reform agenda is gaining momentum with the adoption of a new Public Enterprise Monitoring and Control Act by the National Assembly in September; the introduction of a treasury single account; and the audits of the seven largest public enterprises. New Central 
Bank and Financial Institutions Acts have been put in place, to strengthen the framework for monetary policy and financial sector regulation and development.

Discussions with external creditors aimed at a comprehensive restructuring of Seychelles' unsustainable public external debt are making good progress. The authorities successfully negotiated a debt restructuring with Paris Club creditors in April 2009 and signed a bilateral rescheduling agreements with Malaysia and South Africa on comparable terms. They have also made good progress in talks with private creditors, and a debt exchange offer to holders of external bond, notes, and certain commercial bank loans was launched on December 7, 2009.

\section{Program Summary}

The authorities' program supported by the November 2008 Stand-By Arrangement addressed the foreign exchange distortions, implemented a large fiscal adjustment, and stabilized the macroeconomic environment. The focus has now shifted to responding to the need for a second generation of reforms to transform the economy, secure macroeconomic stability and improve economic performance. The authorities' program for the three years to 2012 under the Extended Fund Facility includes the following targets: (i) average annual real GDP growth of 4-5 percent; (ii) single digit annual inflation; (iii) foreign exchange reserves equivalent to 2.5 months of import coverage by end-2012; and (iv) maintaining a primary fiscal surpluses of about 4.5-7 percent of GDP in the 2010-12 period.

The program includes reforms that focus on:

- Improving public financial management and transparency and governance standards in the public enterprise sector.

- Placing public finances on a firmly sustainable footing through a major reinforcement of fiscal monitoring and control and improved expenditure efficiency, while affecting a strong rise in public investment, which has been neglected during the recent crisis years. A rationalization of the size of the public sector is underway.

- A fundamental medium-term reform of the tax system was launched with the 2010 budget. The objective is to have a simple, fair, and equitable system, which will promote growth, improve self-compliance, and level the playing field for investors.

- Strengthening of the financial sector. The Central Bank of Seychelles (CBS) will implement its action plan for strengthening supervision, and increase minimum capital requirements. The government will also transfer non-bank supervision to the CBS. Steps will also be taken to consolidate and divest publicly owned financial institutions with a 
view to attract strategic investors in the banking sector, bring fresh capital and stimulate competition in the banking sector.

- Restructuring public external debt in a manner that is consistent with the medium-term payments capacity. The authorities aim to secure an immediate and sizable cash flow relief in the short- to medium-term and a sustained reduction of the debt service burden. Significant primary fiscal surpluses are targeted through the medium-term to allow a reduction in domestic debt and provide space for private sector growth.

- Other supporting reforms include the promotion of a better business climate, increased flexibility and efficiency in labor laws, pension reform, improved national statistics, and better sectoral policies for air access, tourism, fishing, and the environment. 
Table 1. Seychelles: Selected Economic and Financial Indicators, 2007-12

\begin{tabular}{|c|c|c|c|c|c|c|c|}
\hline & \multirow[t]{2}{*}{2007} & \multirow[t]{2}{*}{2008} & \multicolumn{2}{|c|}{2009} & \multirow{2}{*}{2010} & \multirow{2}{*}{$\frac{2011}{\text { Projection }}$} & \multirow[t]{2}{*}{2012} \\
\hline & & & Program & Projection & & & \\
\hline National income and prices ${ }^{1}$ & \multicolumn{7}{|c|}{ (Percentage change, unless otherwise indicated) } \\
\hline Nominal GDP (millions of Seychelles rupees) & 6,877 & 8,756 & 10,280 & 10,402 & 11,057 & 11,891 & 12,872 \\
\hline Real GDP & 9.7 & -0.9 & -10.7 & -7.6 & 4.0 & 5.0 & 5.0 \\
\hline $\mathrm{CPI}$ (annual average) & 5.3 & 37.0 & 35.1 & 32.9 & 2.3 & 2.5 & 3.2 \\
\hline CPI (end-of-period) & 16.8 & 63.3 & 5.4 & 1.9 & 2.2 & 3.0 & 3.0 \\
\hline GDP deflator average & 11.4 & 28.4 & 31.4 & 28.5 & 2.2 & 2.4 & 3.1 \\
\hline Money and credit & \multicolumn{7}{|c|}{ (Percentage change, unless otherwise indicated) } \\
\hline Net claims on private sector & 9.3 & 58.4 & 24.3 & -12.1 & 27.3 & $\ldots$ & $\ldots$ \\
\hline Broad money (M3(p)) ${ }^{2}$ & -7.6 & 27.2 & 5.6 & -0.7 & 25.6 & $\ldots$ & $\ldots$ \\
\hline Reserve money & -23.1 & 0.6 & 20.6 & 32.2 & 18.4 & $\ldots$ & $\ldots$ \\
\hline Velocity (GDP/M3) & 1.5 & 1.5 & 1.6 & 1.8 & 1.5 & $\ldots$ & $\ldots$ \\
\hline Money multiplier (M3/reserve money) & 4.2 & 5.3 & 4.7 & 4.0 & 4.2 & $\ldots$ & $\ldots$ \\
\hline Savings-Investment balance & \multicolumn{7}{|c|}{ (In percent of GDP) } \\
\hline External savings & 20.8 & 44.7 & 29.7 & 22.6 & 32.7 & 30.1 & 28.8 \\
\hline Gross national savings & 8.0 & -12.7 & -1.8 & 6.8 & -0.9 & 2.5 & 4.7 \\
\hline Of which: government savings & -4.4 & 4.2 & 1.0 & 2.8 & 2.7 & 5.6 & 7.8 \\
\hline Gross investment & 28.8 & 32.1 & 27.9 & 29.4 & 31.8 & 32.6 & 33.6 \\
\hline Of which: government investment & 5.5 & 2.1 & 4.3 & 5.3 & 6.8 & 7.4 & 8.6 \\
\hline \multicolumn{8}{|l|}{ Government budget } \\
\hline Total revenue, excluding grants & 32.0 & 32.8 & 35.1 & 35.6 & 30.7 & 31.5 & 31.3 \\
\hline Expenditure and net lending & 40.9 & 39.8 & 35.6 & 35.1 & 32.8 & 33.4 & 32.1 \\
\hline Current expenditure & 36.3 & 28.6 & 34.2 & 32.8 & 28.0 & 25.9 & 23.5 \\
\hline Capital expenditure and net lending ${ }^{3}$ & 4.6 & 11.2 & 0.5 & 2.3 & 4.9 & 7.4 & 8.6 \\
\hline Overall balance, including grants & -8.7 & -3.3 & 0.0 & 2.8 & 0.9 & 0.4 & 1.4 \\
\hline Primary balance & -2.0 & 3.8 & 11.4 & 13.3 & 7.0 & 6.0 & 4.7 \\
\hline Total public debt ${ }^{4,5}$ & 129.8 & 135.6 & 149.7 & 140.1 & 109.5 & $\ldots$ & $\ldots$ \\
\hline Domestic & 65.8 & 52.3 & 41.8 & 41.6 & 37.4 & $\ldots$ & $\ldots$ \\
\hline External ${ }^{4,5}$ & 63.9 & 83.3 & 108.0 & 98.5 & 72.2 & $\ldots$ & $\ldots$ \\
\hline External sector & \multicolumn{7}{|c|}{ (In percent of GDP, unless otherwise indicated) } \\
\hline Current account balance including official transfers & -20.8 & -44.7 & -29.7 & -22.6 & -32.7 & -30.1 & -28.8 \\
\hline Total stock of arrears (millions of U.S. dollars) $)^{4,5}$ & 160.4 & 321.7 & 195.1 & 239.2 & $\ldots$ & $\ldots$ & $\ldots$ \\
\hline Total external debt outstanding (millions of U.S. dollars) ${ }^{4,5}$ & 710.2 & 767.1 & 732.6 & 754.7 & $708.1 \ldots$ & & .. \\
\hline (in percent of GDP) ${ }^{4,5}$ & 69.2 & 83.3 & 108.0 & 98.5 & 72.2 & $\ldots$ & $\ldots$ \\
\hline Terms of trade $(=-$ deterioration $)$ & -1.2 & 0.7 & -2.1 & -2.1 & $\ldots$ & $\ldots$ & $\ldots$ \\
\hline Real effective exchange rate (end-of-period, percent change) ${ }^{\circ}$ & -25.4 & -18.4 & $\ldots$ & 13.0 & $\ldots$ & $\ldots$ & $\ldots$ \\
\hline Gross official reserves (end of year, millions of US dollars) & 10 & 51 & 108 & 153 & 202 & 252 & 302 \\
\hline In months of imports, c.i.f. & 0.1 & 0.6 & 1.3 & 1.5 & 1.9 & 2.2 & 2.5 \\
\hline \multicolumn{8}{|l|}{ Exchange rate ${ }^{\circ}$} \\
\hline Seychelles rupees per US $\$ 1$ (end of period) & 8.0 & 16.6 & $\ldots$ & 10.6 & $\ldots$ & $\ldots$ & $\ldots$ \\
\hline Seychelles rupees per US\$1 (period average) & 6.7 & 9.5 & $\ldots$ & 14.1 & $\ldots$ & $\ldots$ & $\ldots$ \\
\hline \multicolumn{8}{|c|}{ Sources: Central Bank of Seychelles; Ministry of Finance; and IMF staff estimates and projections. } \\
\hline \multicolumn{8}{|l|}{${ }^{1}$ Historic GDP data have been revised upwards in April 2009.} \\
\hline \multicolumn{8}{|c|}{${ }^{2}$ In 2007 and earlier, includes domestic currency balances earmarked for pending import requests ("pipeline"). } \\
\hline \multicolumn{8}{|c|}{${ }_{5}^{4}$ Assumes April 2009 Paris Club agreement is implemented; does not include the impact of any further restructuring of public external debt. } \\
\hline
\end{tabular}

Washington, D.C. 20431 • Telephone 202-623-7100 • Fax 202-623-6772 • www.imf.org

CInternational Monetary Fund. Not for Redistribution 


\section{Statement by Christopher Legg, Alternate Executive Director for Seychelles and Simon Duggan, Advisor to Executive Director December 18, 2009}

Our Seychellois authorities' determined adherence to their economic reform program has delivered rapid macroeconomic stabilization, providing a solid base from which to launch a second phase of economic reforms, directed at consolidating macroeconomic stabilization, achieving external debt sustainability and durably raising growth.

\section{Economic Developments and Outlook}

Macroeconomic trends continue to evolve favorably. The nominal exchange rate has appreciated steadily since January and inflation has fallen to around zero. Nominal interest rates on short-term T-bills have declined to under 5 percent, consistent with tight fiscal policy and exchange rate trends. Seychelles' current account deficit has narrowed sharply and the authorities' accumulation of international reserves has exceeded program targets. Real GDP is now expected to contract 7.6 percent in 2009 , somewhat better than the 10.7 percent contraction projected in June.

Seychelles' economic recovery is expected to gain traction from 2010 as the economy responds to the reforms and improving global conditions. There are positive signs that tourism and related construction activity - the main drivers of growth in Seychelles commenced a gradual recovery in the second half of 2009. Real GDP growth is projected to recover to 4 percent in 2010 and to 5 percent in 2011-12, while inflation is expected to remain in the low single digits. The external current account deficit is expected to widen in 2010, due to higher growth and rising foreign direct investment, but narrow over the medium-term as tourism earnings recover. Foreign exchange reserves are projected to rise gradually to about $2 \frac{1 / 2}{2}$ months of import coverage by end-2012.

\section{Program Performance and EFF Request}

Progress on macroeconomic stabilization is underpinned by exemplary program performance. Our authorities met all of the end-September quantitative targets with margins and observed each of the structural benchmarks for the third review. The end-December structural benchmarks are also expected to be met.

Given deep-rooted structural imbalances in the economy, which require sustained reform efforts in the medium-term, our authorities have requested the Fund's continued support for their reform program through a three-year arrangement under the EFF in an amount of SDR 19.8 million (equivalent to 225 percent of quota). The EFF would provide the macroeconomic framework to guide fiscal and financial policies over the next three years, 
bridging Presidential and Parliamentary elections, underpinning the debt reduction strategy and serving a coordinating function for donors and other stakeholders. Our authorities are convinced that the EFF's medium-term, structural focus make it the appropriate instrument to underpin the second phase of their reform efforts.

\section{Fiscal Policy}

Fiscal policy remained tight throughout 2009, out-performing program targets in each of the first three quarters. This provided the space for a supplementary budget of around 2 percent of GDP in the fourth quarter to finance urgent expenditure priorities, while keeping within the annual 2009 primary surplus target of about 131/2 percent of GDP (equivalent to a fiscal tightening in the order of $9 \frac{1}{2}$ percent of GDP in 2009). The government's fiscal performance, coupled with falling interest rates, will finance a reduction in domestic debt of $5 \frac{1}{2}$ percent of GDP in 2009.

Given the rapid macro stabilization, low inflation, improved debt outlook and large development needs, an easing of the fiscal stance to a 7 percent primary surplus is programmed for 2010. The reduction in the primary balance will mainly reflect a fall in revenues associated with one-off positive factors in 2009. The authorities will prudently reduce recurrent spending further as a percentage of GDP in 2010, creating the space to increase capital expenditure to 6.8 percent of GDP, directed primarily at urgent rehabilitation of electricity and water infrastructure. This includes an expenditure contingency of about 1 percent of GDP ear-marked for investment projects that will proceed from the second quarter of the year following confirmation that the fiscal position remains on track.

Going forward, the goals of fiscal policy are to put public finances on a sustainable path while creating the fiscal space to raise public investment. Primary fiscal surpluses will be targeted at 41/2-6 percent of GDP in 2011-12, levels that are consistent with achieving medium-term debt sustainability, assuming full external debt restructuring.

\section{Monetary Policy and the Exchange Rate}

Price stability is the primary objective of monetary policy. The Central Bank's early success in containing inflation and significant appreciation of the rupee provided scope to loosen monetary policy in the second half of 2009. This contributed to a significant reduction in Tbill rates, from over 30 percent at the start of the year to under 5 percent now. However, reflecting the nascent stage of financial sector development, the lending rates of commercial banks have been slow to respond and private sector credit growth remains subdued. The CBS is monitoring credit and price developments closely and stands ready to tighten monetary policy should inflationary pressures re-emerge.

Seychelles is committed to a floating exchange rate regime. The CBS's interventions in the foreign exchange market are limited to smoothing out excessive volatility and ensuring 
orderly market conditions. The establishment of a foreign exchange market has provided a platform for a more efficient allocation of foreign currency among domestic banks; however, institutional factors coupled with market conditions (notably a surplus of foreign exchange in the system) have thus far held back the development of an inter-bank market. The CBS continues to work closely with banks, and make use of technical assistance from the IMF, to improve the efficiency of the foreign exchange market.

\section{Financial Sector}

The CBS continues to strengthen supervision and regulation of the financial sector. A modern Financial Institutions Act was recently put in place, strengthening the framework for financial sector regulation and development. The government also recently transferred supervision of state-owned non-bank financial institutions to the CBS, facilitating more effective oversight. Looking ahead, the CBS continues to implement an 18-month action plan to strengthen risk-based supervision, has plans to increase the minimum paid-up capital requirement for holders of a domestic banking license and will promote the use of the net tangible capital ratio as an added measure of capital adequacy. The CBS is also considering the possibility of introducing a deposit insurance scheme towards the end of the program period to enhance trust of the public in the banking system, and is developing guidelines for a crisis preparedness program.

\section{Structural Reform Agenda}

The government's structural reform agenda is gaining momentum, focused on: fundamental reform of the tax system; rationalizing and reinforcing control over public expenditure; reducing the role of the state in the economy; financial sector development; modernizing labor and pension laws; and improving the business environment.

A fundamental medium-term reform of the tax system was launched with the 2010 Budget. The government's objective is to have a simple, fair, and equitable system, which will promote growth, improve self-compliance and level the playing field for investors. The government's tax reforms have three pillars: business tax rates will be reduced and the business tax base will be broadened by eliminating exemptions and sectoral preferences (tourism and fishing) and reducing thresholds (January 2010); a personal income tax (PIT) will be introduced, broadening the income tax base from withholding on wages to include other domestic-sourced income (July 2010); and a single rate VAT will replace the current GST, broadening the tax base and improving the efficiency of the indirect tax system (January 2012). The tax reform is projected to be broadly revenue neutral over the mediumterm and will be accompanied by efforts to modernize and reinforce revenue administration.

The government is committed to implementing expenditure rationalization measures to create the space for growth-enhancing investment, drawing on the recommendations of World Bank Public Expenditure Reviews. Government employment has been reduced by 
some 2,500 employees (about 15 percent of the public sector workforce) over the last year through a voluntary departure and early retirement scheme. Building on these efforts, the functions of the civil service are being further streamlined, starting with the outsourcing of 650 non-core support staff, the removal of vacant posts from the government payroll and a targeted voluntary departure scheme for the security forces in 2010. The government is now in the process of articulating strategic plans for the health and education sector directed at improving the efficiency of government service provision. Introduction of a Treasury Single Account has reinforced control over public expenditure, while public sector wage policy is also being reformed through the implementation of a new wage grid that will improve transparency and establish a clearer link between remuneration and performance.

The government has adopted a public enterprise reform strategy to make the sector more efficient, transparent and accountable. The legal basis for this work was established with the National Assembly's recent approval of the Public Enterprise Monitoring and Control Act, mandating strengthened reporting arrangements for public enterprises and setting uniform governance rules. In parallel, the government is also taking steps towards further rationalization of public sector ownership, with the Ministry of Finance preparing strategic assessments for each public entity - drawing on recently concluded external financial audits and management audits - evaluating the case for public ownership. The government recently launched tenders for an expert assessment of options for the future of the national airline and has requested IFC help on the privatization of the two publicly-owned banks. The government has also sought the assistance of the FIRST initiative to assess the government's strategic options with regard to the three publicly-owned non-deposit taking institutions with a view to their consolidation and/or privatization.

\section{Public Debt Negotiations}

The Seychelles government has continued its good faith discussions with creditors on a comprehensive public external debt restructuring consistent with their medium-term payments capacity and the Fund's lending into arrears policy. Seychelles successfully negotiated a debt restructuring with the Paris Club in April 2009 and has since signed a bilateral rescheduling agreement with Malaysia on comparable terms. A debt exchange offer to external commercial bondholders was launched in early December, compatible with Seychelles' limited payments capacity and on terms comparable to those agreed with Paris Club creditors. The offer is supported by a guarantee operation from the African Development Bank that will raise the attractiveness for commercial creditors. Our authorities hope to conclude negotiations with commercial creditors early in the new year.

Finally, our Seychellois authorities would like to express their gratitude to Management for their support and the IMF mission chief and his team for their continued hard work in helping to shape Seychelles' economic reform program. 